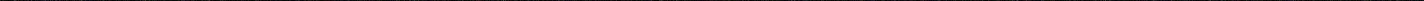




\section{Noise-induced phenomena in the large-scale ocean circulation}


Copyright (C) 2020 by Daniele Castellana

Institute for Marine and Atmospheric research Utrecht (IMAU)

Department of Physics, Utrecht University

Princetonplein 5, 3584 CC Utrecht, The Netherlands

Cover conceived by Martina Huber and Daniele Castellana and designed by Francesca Sciarmella

ISBN: 978-90-393-7329-3

Printed by GVO drukkers \& vormgevers B.V. 


\title{
Noise-induced phenomena in the large-scale ocean circulation
}

\author{
Ruis geïnduceerde verschijnselen in de \\ mondiale oceaancirculatie
}

(met een samenvatting in het Nederlands)

\section{Proefschrift}

ter verkrijging van de graad van doctor aan de Universiteit Utrecht op gezag van de rector magnificus, prof.dr. H.R.B.M. Kummeling, ingevolge het besluit van het college voor promoties in het openbaar te verdedigen op maandag 12 oktober 2020 des middags te 4.15 uur

door

\section{Daniele Castellana}

geboren op 23 oktober 1989

te Larino (Italy) 
Promotor: Prof. dr. ir. H.A. Dijkstra

This work was supported by funding from the European Union's Horizon 2020 research and innovation programme for the ITN CRITICS under Grant Agreement Number 643073. 


\section{Contents}

Summary vii

Samenvatting $\quad$ ix

1 Introduction 1

1.1 Climate variability . . . . . . . . . . . . . . . . . . . . 1

1.2 Dynamical systems framework . . . . . . . . . . . . 3

1.3 Positive feedbacks and tipping points . . . . . . . . . . 6

1.4 Research questions and thesis overview . . . . . . . . . . . 11

2 A statistical significance test for sea-level variability 15

2.1 Introduction . . . . . . . . . . . . . . . . 16

2.2 An alternative motivation for the CAM model . . . . . . . . . . . . . . . . 17

2.2.1 The stochastic shallow-water model . . . . . . . . . . . . . . 17

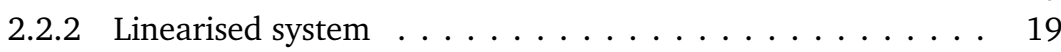

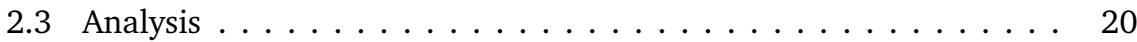

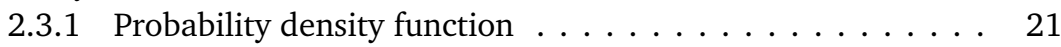

2.3.2 Moments of the distribution . . . . . . . . . . . . . . 22

2.3 .3 Temporal discretization . . . . . . . . . . . . . . . . . 24

2.3.4 Parameter estimation . . . . . . . . . . . . . 25

2.4 Application: Sea-level variability . . . . . . . . . . . . . . . . . . . . . . 29

2.5 Summary and discussion $\ldots \ldots \ldots \ldots \ldots$

3 Model and methods for transition probabilities $\quad 37$

3.1 A box model for the Atlantic Meridional Ocean Circulation . . . . . 38

3.1 .1 Model formulation . . . . . . . . . . . . . . . 38

3.1.2 An indicator for the AMOC stability . . . . . . . . . . . . 41

3.1 .3 Atmospheric freshwater noise . . . . . . . . . . . . . 42

3.2 Transition probabilities of noise-induced transitions . . . . . . . . . . 44

3.2.1 Rare event algorithms . . . . . . . . . . . . . . . . 45

3.2 .2 TAMS algorithm .................. 45

4 The importance of score functions in the TAMS algorithm 49

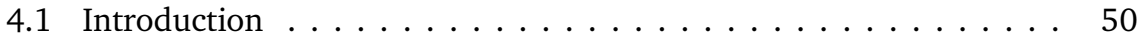


4.2 Methods . . . . . . . . . . . . . . . . . 50

4.2.1 Transition probabilities using TAMS $\ldots \ldots \ldots \ldots \ldots \quad 50$

4.2.2 Different score functions . . . . . . . . . . . . . 51

4.2.3 Definition of the target set . . . . . . . . . . . 52

4.2.4 Estimating the typical transition path using histograms . . . 54

4.3 Results . . . . . . . . . . . . . . . . . . . 55

4.3.1 Incorporating ellipsoids in the score function . . . . . . 56

4.3.2 Designing a score function based on a typical transition path 57

4.3.3 Transition probabilities in a box model of the AMOC . . . . . 61

4.4 Summary and discussion $\ldots \ldots \ldots \ldots \ldots$. . . . . . . 64

5 Transition probabilities of noise-induced transitions of the Atlantic Ocean Circulation 69

5.1 Introduction $\ldots \ldots \ldots \ldots \ldots \ldots \ldots \ldots \ldots \ldots \ldots$

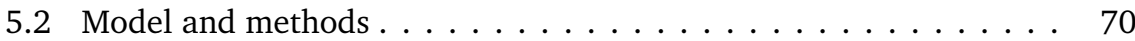

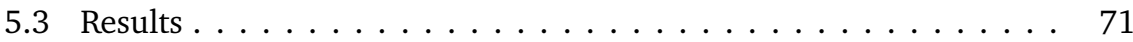

5.4 Summary and discussion $\ldots \ldots \ldots \ldots \ldots \ldots \ldots$

6 Noise-induced transitions of the Atlantic Meridional Overturning Circulation in CMIP5 models 83

6.1 Introduction $\ldots \ldots \ldots \ldots \ldots \ldots \ldots \ldots \ldots \ldots \ldots$

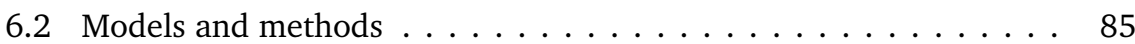

6.3 Results . . . . . . . . . . . . . . . . . . . . 87

6.4 Summary and discussion $\ldots \ldots \ldots \ldots \ldots \ldots$

7 Conclusions and outlook 99

7.1 Null hypothesis for SSH variability . . . . . . . . . . . . . . . 99

7.2 Noise-induced transitions of the AMOC . . . . . . . . . . . 100

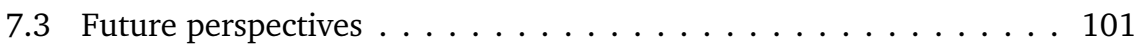

$\begin{array}{ll}\text { Bibliography } & 103\end{array}$

$\begin{array}{ll}\text { Publications and preprints } & 117\end{array}$

$\begin{array}{ll}\text { Curriculum Vitae } & 119\end{array}$ 


\section{List of Figures}

1.1 Artist's view of the climate system variability . . . . . . . . 2

1.2 Dansgaard-Oeschger events in paleoclimate records . . . . . . . 4

1.3 Stommel's box model . . . . . . . . . . . . . . . 7

1.4 Bifurcation, rate and noise-induced tipping $\ldots \ldots \ldots \ldots \ldots$

2.1 Trajectories and histogram SDE with CAM noise $\ldots \ldots \ldots \ldots .23$

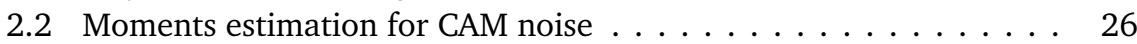

2.3 Fit method estimation for CAM noise $\ldots \ldots \ldots \ldots \ldots \ldots . \ldots 28$

2.4 Red vs CAM noise in SSH global data . . . . . . . . . . . . . . 29

2.5 Null-hypothesis test for location $1 \ldots \ldots \ldots \ldots \ldots \ldots \ldots$

2.6 Null-hypothesis test for location $2 \ldots \ldots \ldots \ldots \ldots \ldots \ldots \ldots$

2.7 Null-hypothesis test for location $3 \ldots \ldots \ldots \ldots \ldots \ldots \ldots$

2.8 Null-hypothesis test for location $4 \ldots \ldots \ldots \ldots \ldots \ldots$

3.1 AMOC box model . . . . . . . . . . . . . . . . . . . . . 39

3.2 Freshwater noise as interannual time scale variability . . . . . . . 43

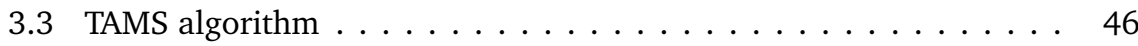

4.1 Double-well potential system . . . . . . . . . . . . . . . 51

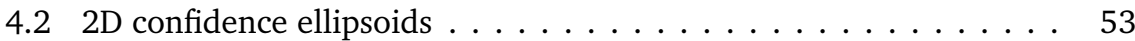

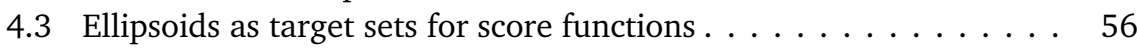

$4.42 \mathrm{D}$ gradient system with wall $\ldots \ldots \ldots \ldots \ldots \ldots \ldots \ldots$

4.5 Typical transition paths and instantons . . . . . . . . . . . 59

4.6 Typical transition path and score function . . . . . . . . . . 59

4.7 Compare transition probabilities with different score functions . . . 61

4.8 Ellipsoids in the AMOC box model . . . . . . . . . . . . 63

5.1 Bifurcation diagrams for AMOC box model $\ldots \ldots \ldots \ldots \ldots$

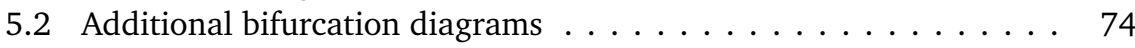

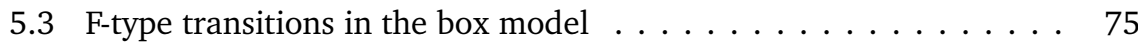

5.4 Transition probabilities of F-type transitions . . . . . . . . . 77

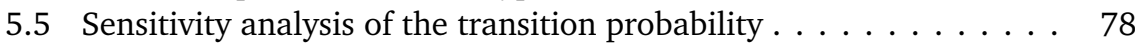

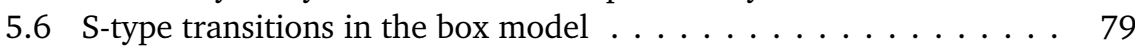

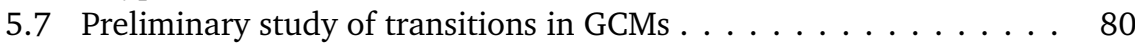


6.1 Wind-stress and buoyancy-induced extremes in the AMOC . . . . . . 88

$6.2 M_{o v}$ in ocean-only and coupled models . . . . . . . . . . . . 89

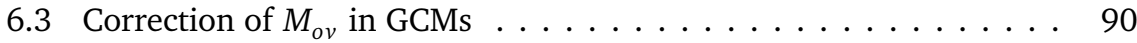

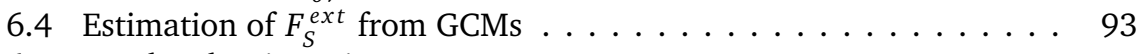

6.5 North Atlantic regions . . . . . . . . . . . . . . . . . . . . . . . 94

6.6 AMOC and North Atlantic temperatures . . . . . . . . . . . . . . 95

6.7 Extreme events synchronisation between AMOC and temperatures . 96

\section{List of Tables}

3.1 Parameters for the AMOC box model . . . . . . . . . . . . . . . . 41

4.1 Parameters for TAMS algorithm with the improved score function . 60

4.2 Advantages of the improved score function for the AMOC box model 64

6.1 List of CMIP5 models and their properties . . . . . . . . . . . . . . 91 


\section{Frequently used acronyms}

$\begin{array}{ll}\text { AMS } & \text { Adaptive Multilevel Splitting (algorithm) } \\ \text { AMOC } & \text { Atlantic Meridional Overturning Circulation } \\ \text { CAM } & \text { Correlated additive and multiplicative (noise) } \\ \text { CMIP5 } & \text { Coupled Model Intercomparison Project 5 } \\ \text { GCM } & \text { General circulation model } \\ \text { PDE } & \text { Partial differential equation } \\ \text { PDF } & \text { Probability density function } \\ \text { SDE } & \text { Stochastic differential equation } \\ \text { SSH } & \text { Sea surface height } \\ \text { SST } & \text { Sea surface temperature } \\ \text { TAMS } & \text { Trajectory-Adaptive Multilevel Sampling (algorithm) }\end{array}$





\section{Summary}

Each research question in climate science requires an appropriate climate model to be formulated, where specific approximations are made and a certain number of physical processes is considered. Conceptual climate models occupy the lowest position in the hierarchy of models: they represent only fundamental processes and they are particularly useful when trying to capture the essence of a certain system. Moreover, when two well-separated time scales can be identified in the system, the processes characterised by the smaller time scale are parametrised, often using a stochastic approach. This is the case, for example, when the ocean circulation is forced by high-frequency atmospheric fluctuations.

This thesis focuses on the effect of atmospheric noise on the large-scale ocean variability, in particular concerning sea surface height (SSH) variability and the stability of the Atlantic Meridional Overturning Circulation (AMOC). Both problems are addressed by using low-dimensional stochastic dynamical systems.

The effect of high-frequency wind-stress variations can be represented as a correlated additive and multiplicative noise (CAM) stochastic model of sea-level variations. The one-dimensional model developed allows to formulate an appropriate null hypothesis for SSH variability: given a time series of sea-level anomalies, specific phenomena in the ocean circulation can be detected by analysing peaks in its power spectrum, and the significance of such peaks can be tested against the null hypothesis. In other words, the model can be used to attribute specific sea-level variability to other effects than wind-stress noise. From the power spectrum analysis of several time series of SSH anomalies in the ocean, we can conclude that the CAM noise process under investigation can explain most of the variability of the sea level. Moreover, we found that some peaks are significant under the traditional red-noise test, but not significant under the CAM noise test. This indicates that using the incorrect test may lead to erroneous attribution of phenomena in the sea-level variability.

Atmospheric noise is not only responsible for the existence of a background signal in the ocean time series. In special kind of systems, noise can lead to the occurrence of tipping points. One of these systems is constituted by the AMOC, which is the zonally integrated volume transport generated by a complex system of currents in the Atlantic Ocean. It represents a crucial component of the climate system, as it redistributes heat northward in the Atlantic. Thanks to the presence of the AMOC, 
north-western Europe experiences a relatively mild climate, compared to maritime regions at similar latitudes on the Pacific (up to $6^{\circ} \mathrm{C}$ warmer). Whether the AMOC is in a multistable regime, i.e. if the system can be in two different states under the same external forcing, is under debate in the climate community. However, if the present-day AMOC were in a multiple equilibria regime, a potential collapse in the near future would cause large and rapid changes in the global climate system. The possibility of occurrence of an abrupt change induced by high-frequency atmospheric variability (i.e. noise) is especially alarming, as noise-induced transitions are inherently unpredictable. In this thesis, we calculate the probability of a noise-induced collapse of the AMOC, given different climate scenarios, by means of a rare-event numerical algorithm. We also suggest some improvements to this algorithm, which make it a promising tool to investigate noise-induced transitions in multistable systems. Lastly, by studying transition probabilities of noise-induced partial collapses of the AMOC in an ensemble of CMIP5 climate models, we revisit one of the stability indicators of the AMOC, i.e. the freshwater transport carried by the overturning circulation at the southern boundary of the Atlantic basin. A correction to this indicator, based on the transition probabilities, is suggested to measure whether an AMOC state is in a multiple equilibrium regime or not. 


\section{Samenvatting}

Voor elke onderzoeksvraag in de klimaatwetenschap is een geschikt klimaatmodel nodig. In dergelijke modellen worden specifieke benaderingen gebruikt en wordt een aantal fysische processen meegenomen. In de hiërarchie van klimaatmodellen staan conceptuele modellen onderaan: ze beschouwen enkel de fundamentele processen en worden vooral gebruikt om de essentie van een bepaald systeem te vatten. Wanneer twee duidelijk verschillende tijdschalen in het systeem kunnen worden geïdentificeerd, worden de processen op de korte tijdschaal daarnaast vaak geparametriseerd met een stochastische methode. Dit is bijvoorbeeld het geval wanneer de oceaancirculatie gedreven wordt door atmosferische fluctuaties met een hoge frequentie. Dit proefschrift gaat over het effect van atmosferische fluctuaties op de grootschalige oceaancirculatie en in het bijzonder over de variatie in het zeeniveau (ZN) en de stabiliteit van de Atlantische meridionale omwentelingscirculatie (AMOC). Voor beide vraagstukken worden laagdimensionele stochastische dynamische systemen gebruikt. Het effect van een snel variërende windforcering kan gerepresenteerd worden door middel van een stochastisch zeeniveaumodel met gecorreleerde additieve en multiplicatieve ruis (CAM). Het in dit proefschrift ontwikkelde eendimensionale model stelt ons in staat om een geschikte nulhypothese op te stellen voor zeeniveauvariabiliteit. Met een tijdreeks van zeeniveauanomalieën kunnen bepaalde fenomenen in de oceaancirculatie worden gedetecteerd. Dit kan door pieken te analyseren in het powerspectrum van de tijdreeks en de opgestelde nulhypothese vervolgens aan dergelijke pieken te toetsen. Met andere woorden: het model kan gebruikt worden om bepaalde zeeniveauvariabiliteit toe te schrijven aan andere effecten dan een stochastische windforcering. Uit de spectraalanalyse van meerdere tijdseries van zeeniveau-anomalieën kunnen we concluderen dat de meegenomen CAM-ruisprocessen het grootste deel van de zeeniveauvariabiliteit kunnen verklaren. Daarnaast constateren we dat sommige pieken significant zijn volgens de rode-ruistest, maar niet volgens de CAMruistest. Dit impliceert dat zeeniveauvariabliteit foutief aan bepaalde factoren kan worden toegeschreven bij het gebruik van de verkeerde test. Atmosferische ruis is niet alleen verantwoordelijk voor het bestaan van een achtergrondsignaal in de oceaantijdseries, maar kan in bepaalde systemen ook leiden tot kantelpunten. Een van dergelijke systemen betreft de AMOC: een zonaal geïntegreerd volumetransport, gegenereerd door een complex systeem van stromingen in de Atlantische 
Oceaan. De AMOC vormt een cruciale component van het klimaatsysteem, omdat het warmte noordwaarts herverdeelt in de Atlantische Oceaan. Dankzij deze circulatie is het klimaat in Noordwest-Europa vrij mild vergeleken met kustregio's op vergelijkbare breedtegraden in de Grote Oceaan (tot 6oC warmer). Of de AMOC zich in een meervoudig stabiel regime bevindt, dat wil zeggen of het systeem in twee verschillende toestanden kan verkeren onder dezelfde externe forcering, is onderhevig aan discussie. Als de huidige AMOC zich in een meervoudigevenwichtsregime bevindt, zou een potentiële overgang naar een ander evenwicht in de nabije toekomst grote en abrupte veranderingen in het mondiale klimaatsysteem teweegbrengen. De mogelijkheid van een dergelijke abrupte verandering, geïnduceerd door atmosferische fluctuaties met hoge frequenties (i.e. ruis), is met name alarmerend doordat deze fluctuaties moeilijk te voorspellen zijn. In dit proefschrift berekenen we de kans op een door ruis geïnduceerde transitie van de AMOC, gegeven verschillende klimaatscenario's met een zogenaamd rare event algoritme. We stellen ook een aantal verbeteringen van dit algoritme voor, die het een veelbelovende methode maken om ruisgeïnduceerde transities in meervoudig stabiele systemen te onderzoeken. Tot slot bestuderen we de kans op een door ruis geïnduceerde partiële transitie van de AMOC in een ensemble van CMIP5klimaatmodellen. Daarmee herzien we een van de stabiliteitsindicatoren van de AMOC, namelijk het zoetwatertransport door de omwentelingscirculatie aan de zuidelijke rand van de Atlantische Oceaan. Om na te kunnen gaan of de AMOC in een meervoudigevenwichtsremige verkeert, suggereren we een correctie van deze indicator gebaseerd op transitiekansen. 


\section{Chapter 1}

\section{Introduction}

\subsection{Climate variability}

The Earth's climate system exhibits variability over a broad range of spatial and temporal scales. In a narrow sense, "Climate is usually defined as the average weather, or more rigorously, as the statistical description in terms of the mean and variability of relevant quantities over a period of time ranging from months to thousands or millions of years. The classical period for averaging these variables is 30 years, as defined by the World Meteorological Organization." [Stocker et al., 2013]. However, a more practical definition includes all time scales of variability (intrinsic and forced) [Dijkstra, 2019].

The traditional view [Mitchell, 1976] of such variability is represented in Fig. 1.1: the power spectrum shown is clearly not directly computed from one time series, but includes information relative to several of them. Indeed, there is no single time series that is over $10^{6}$ years long and with a temporal resolution in the order of one hour. The figure reflects three types of variability [Ghil, 2002]: in the background, a continuous spectrum represents deterministic chaos and stochastically forced variations; on top of it, sharp peaks indicate periodical forcings, whereas broader peaks correspond to internal modes of variability. This classic framework of 'background and peaks' has to be interpreted as a picturesque image of the variability. In fact, it has recently been challenged, as it can only explain a small fraction of the overall variability and the underlying climate system dynamics [see Franzke et al., 2020, for a review]. In particular, over certain spatial and temporal ranges, the actual background spectrum of a climatic time series shows scaling behaviour, which can be described by power laws [Huybers and Curry, 2006, Lovejoy, 2015].

Why are we interested in characterising climate variability? One of the main reason lies in the fact that it allows us to distinguish between phenomena induced by natural processes (e.g. changes in astronomical forcing) from the ones caused 


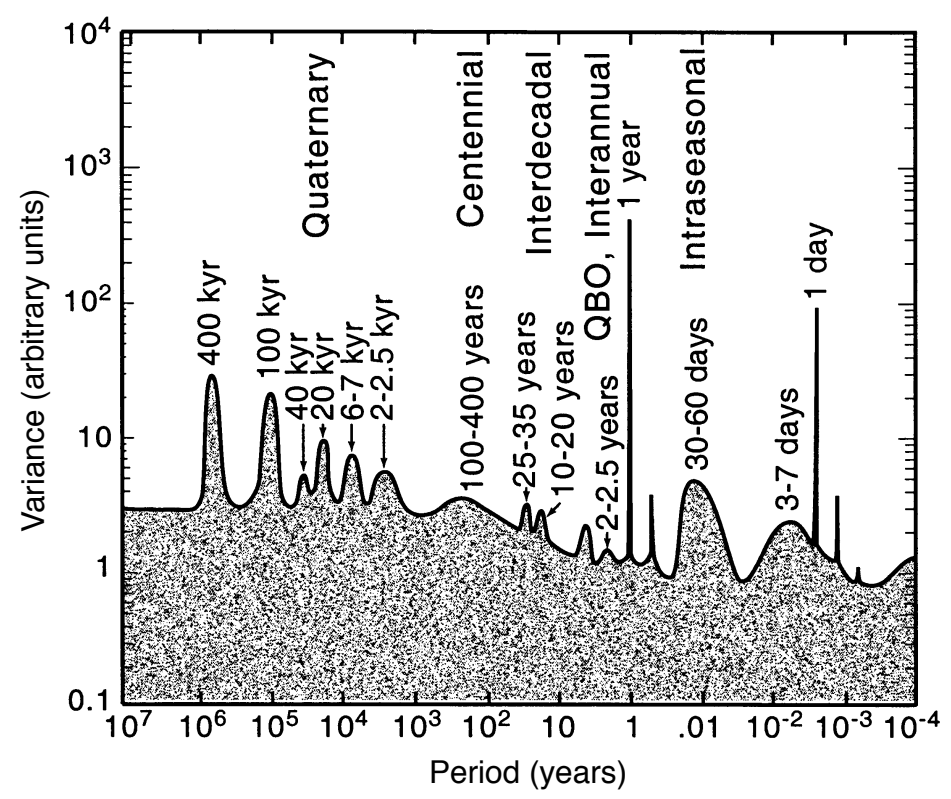

Figure 1.1: Artist's view of the climate system variability over the last $10^{6}$ years [Ghil, 2002], adapted from the original version in [Mitchell, 1976]. The figure represents the traditional 'background and peaks' paradigm of the climate variability. In the last years a new paradigm, based on the scaling behaviour of the variability over certain time scales, was developed [Lovejoy, 2015].

by human activities (e.g. anthropogenic greenhouse gas emissions). Furthermore, increasing our understanding of the physical mechanisms underlying climate variability will ultimately reduce uncertainty and improve our capabilities to predict climate on short and long time scales.

An instructive example of climate variability in the ocean is represented by the sea surface temperature (SST) variability at mid-latitudes. Hasselmann [1976] established a general framework to interpret the basic structure of climate spectra, when two well-separated time scales are identified in the system. High-frequency weather fluctuations (with a decorrellation time scale of approximately one week) are modelled as white noise (with constant power spectrum) acting on the mixed layer of the ocean, which serves as an integrator of the atmospheric signal and, as a result, exhibits low-frequency variability. In mathematical terms, the linear equation governing the evolution of the SST at mid-latitudes [Frankignoul and Hasselmann, 1977] reads

$$
\frac{\mathrm{d} T_{t}}{\mathrm{~d} t}=-A T_{t}+B \eta_{t}
$$


where $T_{t}$ represents the sea surface temperature and $B$ the amplitude of the atmospheric noise. The term $\eta_{t}$ denotes a normally distributed stochastic process, with $\left\langle\eta_{t}\right\rangle=0 \forall t$ and $\left\langle\eta_{t} \eta_{t}^{\prime}\right\rangle=\delta\left(t-t^{\prime}\right) \forall t, t^{\prime}$. The constant $A$ is a damping term, necessary to obtain a statistically stationary response from the ocean; this negative feedback could be represented, for instance, by long-wave radiation fluxes flowing back to the atmosphere. The power spectrum estimated from the solution of the differential equation above is the one of a red noise process, and the equilibrium probability density function (PDF) of the SST is a Gaussian, with mean and variance depending on the magnitude of $A$ and $B$.

When testing this approach on observational data, one finds that, indeed, monthly averaged SST anomalies at mid-latitudes are nearly Gaussian. This simple climate model has been widely adopted as the leading paradigm for the 'null hypothesis' of SST variability in middle and high latitudes. Nonetheless, it is important to stress the importance of the assumptions made by Hasselmann: first of all, the time scales of atmospheric and oceanic variabilities need to be well separated, and no feedback mechanisms are taken into account. Second, eq. (1.1.1) is linear, which is a valid approximation only when non-linear effects are sufficiently weak. Finally, the noise term does not depend on the characteristics of the ocean (i.e. $B$ is a constant) and the noise has a constant power spectrum. We discuss, in the last section of this chapter, the validity of some of these assumptions.

The main focus of this thesis is the effect of atmospheric noise on the largescale ocean variability. Changes in the ocean circulation can considerably influence climate, mainly through their impact on heat transport, which, in turn, alters magnitude and distribution of temperature and precipitation. When looking at millennial time scales, drastic changes in the Atlantic circulation are believed to be responsible of climate shifts during the last glacial periods [Clement and Peterson, 2008, Crucifix, 2012]: such shifts are referred to as Dansgaard-Oeschger (DO) events [Dansgaard et al., 1993] and are most pronounced in the Greenland ice core records (Fig. 1.2). The temperature difference between warm (interstadial) and cold (stadial) periods is believed to have reached up to $16^{\circ} \mathrm{C}$ [Kindler et al., 2014].

\subsection{Dynamical systems framework}

Dynamical system theory is a powerful framework for studying asymptotic states of the climate system. Thanks to the separation of time scales, like the one presented in the previous section, it is possible to attribute climate variability phenomena to low-order behaviour [Dijkstra, 2019].

Depending on the range of temporal and spatial scales of interest, and hence on the number of physical processes considered, one can describe the climate system with a dynamical system of a certain complexity and number of degrees of freedom. Conceptual climate models consist of a small set of ordinary differential equations (ODEs) and only fundamental processes are considered, which capture 


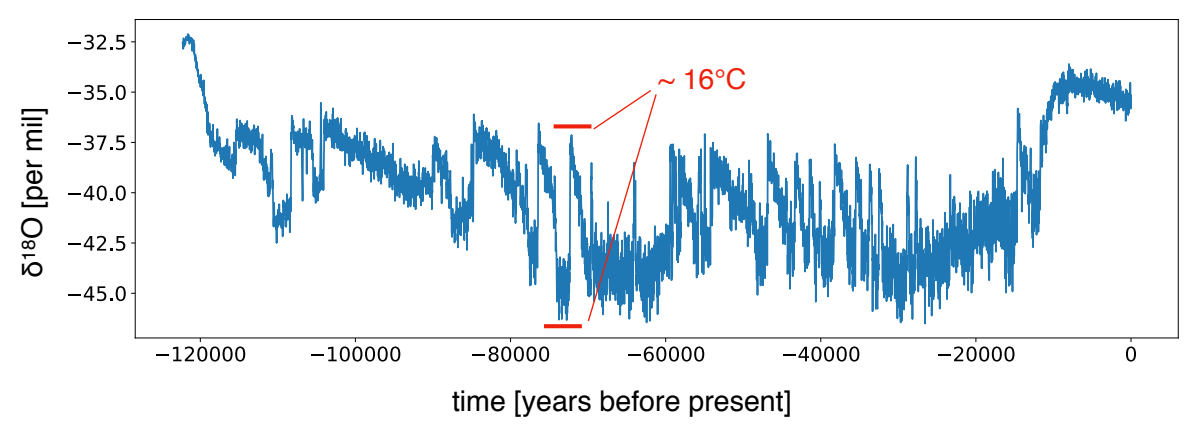

Figure 1.2: Temperature proxy from the Northern Greenland Ice-core Project (NGRIP) core [Dansgaard et al., 1993]. The Dansgaard-Oeschger events are fluctuations between two different states, referred to as stadials (corresponding to a cold climate) and interstadials (corresponding to a warm climate). The temperature difference between the two states was estimated to take values up to $16^{\circ} \mathrm{C}$ [Kindler et al., 2014]. These events are believed to be related to major changes in the Atlantic Ocean Circulation during the last glacial period and, hence, suggest the existence of multiple steady states in the Atlantic circulation.

the essence of the system. Prominent early examples are the model for atmospheric convection developed by Lorenz [1963], the Stommel box-model of the Atlantic Meridional Overturning Circulation (AMOC) [Stommel, 1961], and the SST model studied by Hasselmann, described in the previous section. When the spatial extension of the system is taken into account, and more processes are included, intermediate complexity models are formulated: they are described by a set of partial different equations (PDEs), as many as the number of the variables in the system. PDEs, equipped with appropriate boundary and initial conditions, can be discretised by means, for example, of finite difference methods, and, eventually, a set of ODEs with algebraic constraints can be written [Pedlosky, 1996]. The highest position of this hierarchy of models is occupied by the so-called Earth system models (ESMs), or general circulation models (GCMs), which integrate the interactions of atmosphere, ocean, land, ice, and biosphere. Despite being extremely simplified, conceptual models have the great advantage of isolating key processes of a certain climate subsystem, facilitating their understanding. Moreover, unlike complex models, the computational power required to simulate their time evolution is generally modest: therefore, they easily allow for ensemble simulations and long runs, which are particularly useful when investigating climate variability on long time scales.

In order to formulate a climate model, specific approximations are made, which need to be adequate for the research questions one wants to address. For example, to predict the weather in the Netherlands over the next two weeks, one can certainly assume a constant eccentricity of the Earth's orbit. Similarly, when studying the Dansgaard-Oeschger events, local fluctuations of the temperature appear irrel- 
evant. When formulating a dynamical system to represent a certain phenomenon, common practice is to consider all processes with time scales much greater of the time scale of interest as fixed in time, whereas a different treatment is needed for the ones which are much smaller than that. Generally, small spatial and time scales are parametrised, using either a deterministic or a stochastic approach. Hasselmann's theory, presented in the previous section, is an example of stochastic parametrisation of a small time scale atmospheric forcing. The main assumption behind this procedure is that the lag- 1 autocorrelation of the parametrised phenomena is negligible. Therefore, a deterministic autonomous system becomes a non-autonomous one, where noise is imposed. Such systems are called stochastic dynamical systems and consist of a set of ordinary (or partial) stochastic differential equations (SDEs) [Gardiner et al., 1985], in the form

$$
\mathrm{d} \mathbf{X}_{t}=F\left(\mathbf{X}_{t}\right) \mathrm{d} t+G\left(\mathbf{X}_{t}\right) \mathrm{d} \mathbf{W}_{t},
$$

where $\mathbf{X}_{t} \in \mathbb{R}^{n}$ represents the state of the system at time $t, F: \mathbb{R}^{n} \rightarrow \mathbb{R}^{n}$ is the deterministic term (often referred to as drift field), $G: \mathbb{R}^{m} \rightarrow \mathbb{R}^{n}$ is a map that defines the coupling of the system with the noise. The term $\mathbf{W}_{t} \in \mathbb{R}^{m}$ denotes a multivariate Wiener process (or Brownian motion, in one dimension). The equation above can be further generalised, for instance introducing a time dependence in $F$ or $G$, or by adding algebraic constraints to the system. For an SDE to be properly defined, we need to specify whether its solution is formulated following either Itô or Stratonovich interpretation [Gardiner et al., 1985]. A complete description of the two approaches lies outside the scope of this thesis. It is worth to mention that the Stratonovich SDEs are the most used in physical systems, as they can be seen as limits of continuous time processes with increasingly small correlation times [van Kampen, 1981, Soldatenko and Yusupov, 2017].

The last important aspect to explore here is the nature of the noise term in eq. (1.2.1). Different physical processes are parametrised with different types of noise. The original Langevin equation [Langevin, 1908], historically the first example of a stochastic differential equation, describes the motion of a particle in a fluid: the random collisions with the molecules of the fluid can be modelled by adding white noise to the system. Mathematically, this can be achieved by setting $G$ constant in eq. (1.2.1). Additive white noise has been widely used when formulating stochastic atmospheric models [e.g. Egger, 1981, Delsole and Farrell, 1995, Newman et al., 1997, Whitaker and Sardeshmukh, 1998]. However, if the stochastic forcing represents fluctuations of parameters due to unresolved smallscale processes, it is often state-dependent, and referred to as multiplicative noise [Neelin and Weng, 1999, Sardeshmukh et al., 2001, Sura, 2002, Lin and Neelin, 2002, Monahan, 2004]. Moreover, as we have seen in the previous section, climate variability is not equal at all frequencies: therefore, there exist processes that can be better modelled as red noise [Pierini, 2010], for instance. Eq. (1.2.1) can be easily adapted to allow for the presence of coloured noise. Finally, it is worth to mention that the classic stochastic calculus can be extended to include other kind 
of processes in addition to a Wiener process. Levy flights [Applebaum, 2009], for example, might be responsible for climate changes observed in paleoclimate records [Ditlevsen, 1999]. In this work, we only consider noise originating from high-frequency atmospheric variability.

\subsection{Positive feedbacks and tipping points}

A prominent example of the potential of conceptual models for the understanding of key climate processes is Stommel's box model of the Atlantic Ocean Meridional Circulation [Stommel, 1961]. The Atlantic Ocean Circulation has a special role in the climate system, because its associated meridional heat transport is positive over all latitudes [Srokosz et al., 2012], peaking at about $1.5 \mathrm{PW}$ at $26.5^{\circ} \mathrm{N}$ [Johns et al., 2011]. Responsible for this heat transport is the Atlantic Meridional Overturning Circulation (AMOC) which is the zonally integrated volume transport generated by a complex system of Atlantic Ocean currents. Thanks to the release of heat from the ocean to the atmosphere in the North Atlantic, northwest Europe experiences a relatively mild climate, respect to maritime regions at similar latitudes on the Pacific (up to $6^{\circ} \mathrm{C}$ warmer) [Palter, 2015].

Stommel proposed a minimal model for the AMOC, intended to analyse the effect of surface freshwater and heat fluxes on the large-scale two-dimensional (zonally integrated) ocean circulation in the Atlantic (Fig. 1.3(a)). Two boxes, representing the equatorial and the polar regions, respectively, are connected by means of an overflow channel at the surface and a capillary tube at the bottom. The water in each box is well mixed, so that temperature and salinity are uniform inside it. A linear equation of state is assumed, and the flow rate, directed from high to low pressures, is proportional to the density difference between the two boxes. The exchange between each box and the atmosphere is modelled through a relaxation to prescribed values of temperature and salinity. Since the prescribed salinity at the equator is higher than the one at the pole, a strengthening in the circulation results in more salt being transported towards the polar box. As the flow rate is proportional to the density difference, and hence to the salinity difference, an enhanced salinity at the pole leads, in turn, to a further intensification of the circulation. This positive feedback is referred to as the salt-advection feedback. On the other hand, if one investigated the response of the temperature to a perturbation in the flow, would find a negative feedback. Nonetheless, perturbations in the temperature are more easily damped by the interaction with the atmosphere, thanks to the fast time scale at which the relaxation to the prescribed temperature occurs. In mathematical terms, the salt-advection feedback can be explained by the non-linearity of the governing equations of the box model, which, under a 
(a)

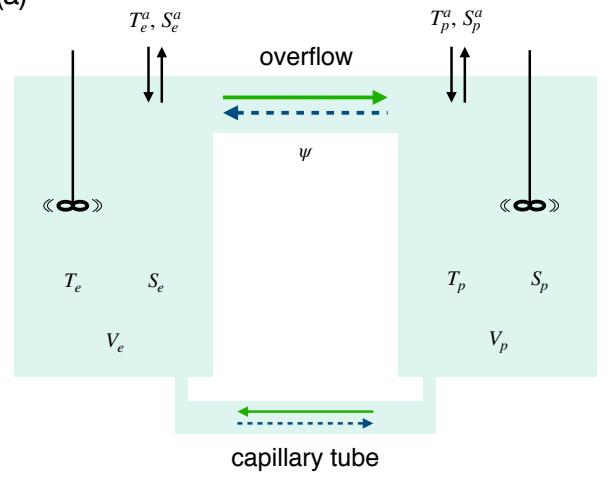

(b)

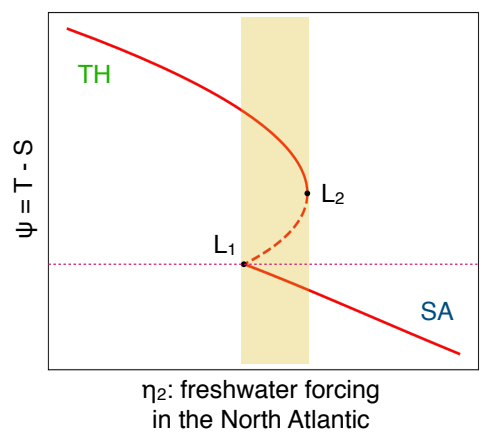

Figure 1.3: (a) Stommel's box model of the AMOC [adapted from Stommel, 1961]. The climate variables with subscript $e(p)$ are relative to the equatorial (polar) box; the superscript $a$ indicates the prescribed values of temperature and salinity in the atmosphere. The green solid arrows indicate the direction of the circulation when thermally driven $(\psi>0)$, whereas the blue dash arrows represent the salinity-driven circulation $(\psi<0)$. (b) Bifurcation diagram for the model in (a). The variable on the $y$-axis is the non-dimensional flow rate $\psi$, computed by subtracting the salinity difference between the two boxes $\left(S_{e}-S_{p}\right)$ from the temperature difference (with the same convention of signs). The dotted magenta line indicates zero flow rate. Solid lines denote stable solutions, respectively the thermallydominated (TH) solution and the salinity-dominated one (SA), while the dash line indicates the unstable steady state. The yellow band highlights the bistable region, bounded by two saddle-node bifurcation points $L_{1}$ and $L_{2}$.

suitable scaling, read

$$
\begin{aligned}
& \frac{\mathrm{d} T}{\mathrm{~d} t}=\eta_{1}-T(1+|T-S|), \\
& \frac{\mathrm{d} S}{\mathrm{~d} t}=\eta_{2}-S\left(\eta_{3}+|T-S|\right) .
\end{aligned}
$$

In the equations above, $T$ and $S$ represent, respectively, the differences of the temperatures and the salinities between the two boxes (equatorial minus polar). The parameters $\eta_{1}$ and $\eta_{2}$ are a measure of the strength of the thermal and freshwater atmospheric forcing, respectively, while $\eta_{3}$ is the ratio between the thermal and the freshwater relaxation time scales. Thanks to the non-linear terms $T|T-S|$ and $S|T-S|$, the system exhibits multiple stable states, for a suitable choice of the parameters. In particular, this occurs when the salt-advection feedback is strong enough, hence when the two relaxation time scales are well separated. In this case, Fig. 1.3(b) shows the sensitivity of the flow rate $\psi$ to the freshwater forcing $\eta_{2}$. When the forcing is small, a unique stable equilibrium is found, which takes the name of thermally-dominated (TH) state, as $\psi=T-S>0$. On the contrary, for 
high values of $\eta_{2}$, there exists a salinity-dominated (SA) state, with $\psi=T-S<0$. At intermediate values, the two states coexist, and the system shows multistability: that means that, for the same choice of the parameters, the system can asymptotically reach two different states, depending on the initial conditions imposed. The dashed curve in Fig. 1.3(b) indicates the presence of an unstable equilibrium, or saddle. Diagrams like the one represented in the figure are computed by finding equilibrium solutions of systems like the one in eq. (1.3.1) - more in general, imposing $F(X)=0$ in the deterministic system obtained from the generic SDE (1.2.1) - by means of continuation techniques [Keller, 1977].

They are called bifurcation diagrams, as they give a visual representation of the bifurcations in a dynamical system. A bifurcation is a qualitative change in the solutions of the system, in terms of their type or number. Two examples are shown in Fig. 1.3(b): both $L_{1}$ and $L_{2}$ take the name of saddle-node bifurcation points, characterised by the sudden appearance or disappearance of one or more solutions when varying a certain parameter. Other examples of saddle-node bifurcations in climate dynamics include, for example, energy balance models [Ghil, 1976, North et al., 1983, Charney and Devore, 1979]. Dynamical systems can exhibit a plethora of bifurcations [Guckenheimer and Holmes, 2013], which go from changes in stability properties of fixed points (pitchfork, transcritical), to the occurrence of oscillatory behaviour (Hopf), to the collective interaction of multiple instabilities, that leads to global bifurcations. For a brief collection of climate variability phenomena described by several types of bifurcation, see [Dijkstra and Ghil, 2005].

Coming back to the Stommel's box model, the presence of multistability can lead to the occurrence of critical transitions. For instance, imagine that the state of the system is located in the multistable region, on the TH branch of the diagram 1.3(b). A sudden shift to the SA solution can take place because of two different causes: (i) when the parameter $\eta_{2}$ is slowly increased, until it crosses the saddlenode point $L_{2}$; (ii) if noise is added to eqs. (1.3.1). Depending on the underlying cause, the abrupt change in the system takes the name of bifurcation or noiseinduced transition, respectively. Other systems can exhibit another kind of abrupt transitions, occurring when the control parameter is increased at a high rate, so that the state of the system is not able to track its equilibrium state: they take the name of rate-induced tipping [Wieczorek et al., 2011]. The three cases are depicted in Fig. 1.4.

Such critical events are often referred to as 'tipping' phenomena. Lenton et al. [2008] listed a series of tipping elements in the Earth's climate system, which could potentially undergo a transition due to the occurrence of instabilities. Greenland Ice Sheet [Ridley et al., 2010, Robinson et al., 2012], Indian Monsoon [Sultan and Janicot, 2000] and Amazon Rainforest [Higgins and Scheiter, 2012, Lasslop et al., 2016] are notable examples among them, in addition to the AMOC. At this point, it appears natural to ask whether a conceptual dynamical system like the Stommel's box model can actually represent the real oceanic circulation, or at least give a qualitative picture of it. Numerous studies have addressed this problem in the 
(a)

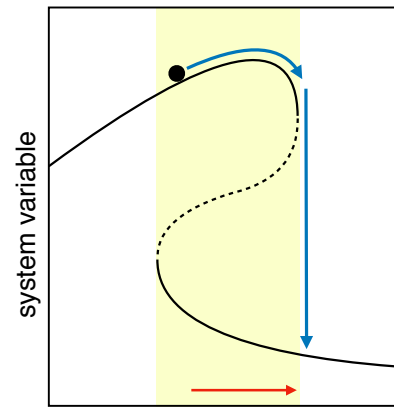

control parameter (b)

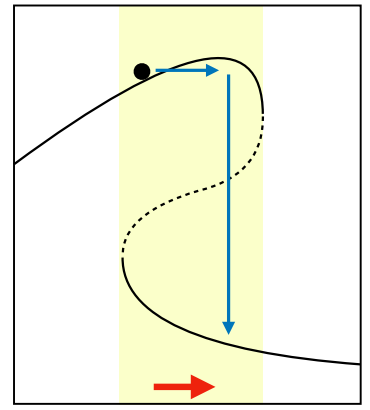

control parameter (c)

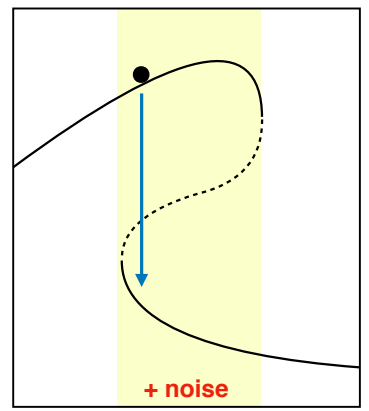

control parameter

Figure 1.4: Typical bifurcation diagram for a system that can exhibit three types of tipping behaviour. The initial state of the system is represented by the black circle. Red arrows indicate magnitude (length) and rate (thickness) of the increase of the control parameter; blue arrows indicate the trajectory of the system in the parameter-variable space. The yellow bands highlight the bistable regions, each of them bounded by two saddle-node bifurcation points. (a) Bifurcation-induced tipping: the control parameter is slowly increased, until it crosses a saddle-node bifurcation point. The state of the system tracks the upper equilibrium branch of the diagram, until it falls to the lower branch. (b) Rate-induced tipping: the rate of variation of the parameter is high, to the extent that the state is no longer able to track the upper branch, and the system undergoes a transition to the lower steady state. (c) Noiseinduced tipping: the control parameter is kept constant, while noise is added to the system, causing the occurrence of a transition.

last 60 years, and several models with different levels of complexity have shown bifurcation diagrams similar to the one in Fig. 1.3(b) [see chapter 5 of Dijkstra, 2005, for a collection of studies]. The highest model in the complexity hierarchy, for which a similar structure was found, is an intermediate complexity model, that combines a global ocean model with an energy balance atmospheric model [den Toom et al., 2012]. Similar studies have been carried out on other tipping components of the Earth systems (such as the ones already mentioned); for a review of climate and eco-systems that exhibit bifurcations in a hierarchy of models, see [Bathiany et al., 2016]. Bifurcation diagrams are generally not available for fully coupled GCMs: that means that long - and computationally expensive - simulations need to be run in order to detect abrupt changes in the corresponding time series. Sudden collapses of the AMOC, as a response to slow increases of the freshwater forcing in the North Atlantic, have been found in several ocean and climate models [de Vries and Weber, 2005, Hawkins et al., 2011, Vellinga and Wood, 2002, Rahmstorf et al., 2005]. Nonetheless, such abrupt changes are not easy to find in state-of-the-art global climate models, such as the ones used in the fifth assessment report (AR5) of the Intergovernmental Panel for Climate Change (IPCC). Drijfhout et al. [2015] made an attempt in this direction: they systematically searched for 
evidence of abrupt changes in the simulations produced by Coupled Model Intercomparison Project 5 (CMIP5) [Taylor et al., 2012], that were used to inform the IPCC, and found them in sea ice, oceanic flows, land ice, and terrestrial ecosystem response, although with little consistency among the models.

No transitions were found in the AMOC in CMIP5 models. This might indicate that the multistability of the circulation might be an artefact of the simple models, because of the absence of the stabilising effect of the atmospheric feedback [Yin et al., 2006]. However, den Toom et al. [2012] have shown that such feedbacks are not strong enough to eliminate the multistability. On the other hand, other studies support the idea that the Atlantic Circulation may be much more sensitive to freshwater anomalies than what the global climate models show [Drijfhout et al., 2011]. Furthermore, the occurrence of DO events during the last glacial period hints at the existence of multistable states in the Atlantic Ocean Circulation. The mechanisms involved include stochastic resonance [Ganopolski and Rahmstorf, 2002] and noise-induced transitions [Ditlevsen et al., 2005].

As already mentioned, the stability of the states of a complex model, especially high up in the model hierarchy, are generally not known. Therefore it is not possible, for a system which could potentially exhibit multistability, to know whether the current state lies in the multistable region. Nevertheless, Rahmstorf [1996] defined a criterion for the bistability of the AMOC, $M_{o v}$, that is the sign of the AMOC freshwater transport at the southern boundary of the Atlantic Ocean, when the system is in the present-day state (corresponding to the TH solution in the Stommel model). It is remarkable that an observable calculated from one equilibrium state of the system can give information about the existence of another steady state. The reasons behind the validity of the indicator, which are discussed in detail in the next chapters, are based on specific physical mechanisms, inherent to the AMOC.

Even if a certain system is known to be in a multistable regime, it is generally not possible to know whether a bifurcation point is nearby. As a result, predicting bifurcation-induced transitions is a serious challenge. Early-warning signals have been developed, in order to detect the approach of tipping points: the underlying idea is that a system close to a critical transition becomes increasingly slow in recovering from small perturbations. This phenomenon is known as 'critical slowing down' and has been widely used to analyse time series of ecological [Scheffer et al., 2009] and climate systems [Lenton, 2011]. When slowing down occurs, the time series often show an increase in auto-correlation [Held and Kleinen, 2004, Dakos et al., 2008] and variance [Ditlevsen and Johnsen, 2010, Carpenter and Brock, 2006]. More advanced measuring techniques include degenerate fingerprinting [Held and Kleinen, 2004] and detrended fluctuation analysis [Livina and Lenton, 2007]. Nevertheless, most of these statistical indicators are sometimes difficult to calculate with statistical significance [Boettiger et al., 2013], and they seem to be not reliable when dealing with bifurcations other than the saddle-node [Dutta et al., 2018]. When time-dependent forcing is considered [Wieczorek et al., 
2011], delayed early-warning signals have been found for rate-induced transitions [Ritchie and Sieber, 2016].

On a different note, noise-induced transitions do not show any of the earlywarning signals mentioned above [Lenton, 2011], thus they are generally unpredictable [Ditlevsen and Johnsen, 2010]. In systems with a high level of noise, flickering between states (i.e., noise-induced transitions in both directions) may occur prior to a more permanent transition [Bakke et al., 2009]. Furthermore, transition probabilities of abrupt changes in paleoclimate records can been estimated from long time series, by inferring the stability landscape of the system and the noise amplitude from the analysis of several transitions [Livina et al., 2011]. However, there are two main limitations in these approaches: first of all, they require the knowledge of both the steady states involved in the transition, which, as already discussed, is often not available; second, even when the equations of the underlying dynamical system are known, one would like to find a systematic method to estimate transition probabilities for general (non-gradient) systems, also when such probabilities are small and no transitions have been observed yet.

\subsection{Research questions and thesis overview}

This thesis investigates the role of the atmospheric noise in the large-scale ocean circulation, in particular with regard to the sea surface height (SSH) variability and the stability of the AMOC.

In section 1.1 spectral analysis was introduced as an effective tool for investigating the mechanism underlying climate variability. In particular, given a time series of a certain observable, specific phenomena can be detected by analysing peaks in its power spectrum. In order to test the significance of such peaks, one needs to formulate an appropriate null hypothesis: indeed, as already discussed, part of the variability observed in every climate time series is generated by the response to high-frequency deterministic and stochastic forcing. In the case of SST variability at mid-latitudes, Hasselmann's model provides a null hypothesis based on a red-noise process as a background signal. The test has been successfully applied in many studies [Mann et al., 1999, Serra et al., 2001, Newman, 2007, among others], in order to detect low-frequency variability phenomena in the ocean circulation. We already mentioned some of the assumptions of Hasselmann's approach, among which the presence of a stochastic forcing that does not depend on the climatic variable (in this case, SST). This choice makes the test of the null-hypothesis straightforward, but it is not necessarily the correct one, especially when the distribution of the climate variable under study is not Gaussian. 
Sea level height (SSH) time series, for instance, often show non-Gaussian distributions [Thompson and Demirov, 2006] and a linear stochastic model with additive white noise does not represent an appropriate null-hypothesis test for the associated variability. Sura and Gille [2010] proposed a new paradigm for SSH variability, based on a linear model with multiplicative noise. Nevertheless, a statistical significance test has not been formulated yet. The first research question of the present work is the following:

1. What is an appropriate null-hypothesis test for SSH variability, considering that multiplicative noise comes into play, via the interaction of the ocean and the atmospheric forcing?

In chapter 2 the question is addressed. A one-dimensional stochastic model is formulated, based on a shallow water model of the ocean circulation; the characteristics of the resulting correlated additive and multiplicative (CAM) noise are described in details and a new statistical test, with parameters estimated from a SSH time series, is developed.

The second research line of this work revolves around noise-induced transitions in multistable systems, discussed in section 1.3. In particular, we concentrate on the AMOC. By reviewing the main literature concerning its multistability, we can conclude that obtaining the correct AMOC stability in climate models is a great challenge and the debate around it is not settled [Gent, 2018]. However, if the present-day AMOC were in a multiple equilibria regime, a potential collapse in the near future would cause large and rapid changes in the global climate system [Jackson et al., 2015]. The possibility of occurrence of an abrupt change induced by high-frequency atmospheric variability (i.e. noise) is especially alarming, as noise-induced transitions are inherently unpredictable. The main question we are interested in is the following:

2. What is the transition probability of a noise-induced collapse of the AMOC under current atmospheric variability?

We have seen that, for several models of the AMOC of various complexity, the bifurcation diagram of the system is known. Therefore, transition probabilities of transitions between different stable states can, in principle, be computed, by using, for example, Monte Carlo simulations. Nevertheless, when the probabilities involved are very small and/or the model under study high-dimensional, more sophisticated methods need to be implemented [Lestang et al., 2018]. In chapter 3 we discuss in detail one of this methods, namely the Trajectory-Adaptive Multilevel Sampling (TAMS). In the same chapter, we describe a box model for the AMOC, originally formulated by Cimatoribus et al. [2014], that is used in the next chapters. In chapter 4 we introduce some improvements to the TAMS algorithm, necessary to make it suitable for computing transition probabilities between steady states. We then calculate transition probabilities for noise-induced transitions of the AMOC in 
the next 100 years, using the aforementioned box model: the results, presented in chapter 5, show that two kinds of transitions can occur, corresponding to a partial and full collapse of the AMOC, respectively, with different transition probabilities. In order to compare these results to those of GCMs, for which bifurcation diagrams are not available, we make use of the above-mentioned indicator for the stability of the AMOC, i.e. $M_{o v}$. Probabilities are then computed for a range of realistic values of $M_{o v}$ and atmospheric forcing. Finally, in chapter 6, we investigate AMOC transitions (i.e. partial collapses) in an ensemble of CMIP5 climate models: by relating the associated probabilities to the values found in the box model, we revisit the idea behind the use of $M_{o v}$, and suggest a correction to the indicator, so that it can become suitable for GCMs. The results further demonstrate the dependence of the Atlantic surface freshwater flux on the AMOC and the impact of extreme events in the AMOC on temperatures in the North Atlantic region. The thesis in concluded with a recap of the main results and future perspectives in chapter 7 . 



\section{Chapter 2}

\section{A statistical significance test for sea-level variability}

A statistical test is presented to address the null hypothesis that sea-level fluctuations in the open ocean are solely due to additive noise in the wind stress. The effect of high-frequency wind stress variations can be represented as a correlated additive and multiplicative noise (CAM) stochastic model of sea-level variations. The main novel aspect of this study is the estimation of parameters in the discrete CAM model from time series of sea surface height observations. This leads to a statistical test, similar to the red noise (or AR(1)) test for sea surface temperature, which can be used to attribute specific sea-level variability to other effects than wind-stress noise. We demonstrate the performance of this test using altimeter data at several locations in the open ocean.

This chapter has been published as:

Castellana, D., Dijkstra, H. A., and Wubs, F. W. (2018). A statistical significance test for sea-level variability, Dynamics and Statistics of the Climate System, 3(1), dzy008. 


\subsection{Introduction}

Sea level varies on many temporal and spatial scales [Harrison, 2002]. Tide gauges and altimetry are currently providing most of the instrumental data; some of the tide gauge records are over 100 years long while the altimetry data record starts in 1992. This study is motivated by the problem of attributing sea surface height (SSH) variations to specific mesoscale ocean features, such as eddies [Firing and Merrifield, 2004] or rings [Beal et al., 2011]. This is problematic as much of the sea-level variability is due to other processes, for example, due to high-frequency wind-stress fluctuations and Rossby waves [Hughes and Williams, 2010].

For sea surface temperature (SST) variability, a similar problem occurs of distinguishing SST changes due to specific large-scale phenomena (e.g. El Niño) from those caused by high-frequency fluctuations in atmospheric temperature and the associated heat fluxes. This problem was addressed over 40 years ago by Hasselmann [1976], who introduced a stochastic model of the ocean mixed layer. In this model, the SST anomalies can be modelled by an Ornstein-Uhlenbeck process with a Gaussian probability density function (PDF). The discrete variant of this process is the AR(1) or red noise process which is serving as a null hypothesis for SST variability. Indeed, when applied to SST variability in the Eastern Tropical Pacific, the El Niño variations are such that this null hypothesis can be rejected [Neelin et al., 1998].

In a series of studies, extensive statistical analyses of daily observed SST [Sura et al., 2006, Sura and Sardeshmukh, 2008] and SSH [Sura and Gille, 2010] variations were performed. There are many areas of the globe where the skewness (S) and excess kurtosis (K) values of SST anomaly time series are far from Gaussian values $(S=K=0)$. Examples are large areas in the Southern Ocean (e.g. $S \sim-0.4$ ) and the Eastern Tropical Pacific (e.g. $S \sim 0.4$ ), with also substantial seasonal dependence. Much larger deviations from Gaussian distributions are found in SSH anomaly time series [Sura and Gille, 2010], where $S$ values range from -2 to +2 . Analysis of probability density functions for SSH indicates that these have a piecewise power law behaviour, also suggested in earlier studies [Thompson and Demirov, 2006]. In [Hughes and Williams, 2010], it was found (using 12 years of altimetry data) that an AR(5) spectral fit provides an adequate representation of the shape of the spectrum over much of the ocean; this information was used for trend (rate of sea-level change) detection. In [Bos et al., 2014], the effect of the specific statistical models on the error in the estimated sea-level trend was calculated.

To explain the non-Gaussian behaviour of SST variability, the stochastic Hasselmann model (from now on, red noise model) was extended to include (in addition to the additive noise due to the heat flux) a multiplicative noise term, due to the dependence of the heat flux noise on the SST anomaly [Sura et al., 2006, Sura and Sardeshmukh, 2008]. The resulting model of Correlated Additive and Multiplicative (CAM) noise can be analysed through its equilibrium probability density function (PDF) by solving the corresponding Fokker-Planck equation. It was 
shown [Sura and Sardeshmukh, 2008, Sardeshmukh and Sura, 2009] that this model gives a power law PDF for which $K \geq 3 S^{2} / 2$, in agreement to what is found from SST observations. A CAM noise stochastic model was also used to explain the non-Gaussian behaviour of SSH anomalies [Sura and Gille, 2010] and to show why $K>3\left(S^{2}-1\right) / 2$ for most locations on the globe.

From all these studies, it is clear that the CAM noise stochastic model may serve as a null hypothesis, in particular for SSH variability, where the deviations from Gaussian behaviour are much larger than that for SST. While the use of such a null hypothesis is mentioned in [Sura and Gille, 2010] this has, to our knowledge, not yet resulted in a statistical test which can be used to reject it. Such a test would replace the red noise test which is often carried out to draw conclusions about the significance of certain frequencies of SSH variability.

The main purpose of this study is to develop a statistical test for wind-stress driven sea-level variability based on a stochastic model for which the parameters can be estimated from the altimetry (or tide gauge) time series (like the lag- 1 autocorrelation in the AR(1) process). In section 2.2, we present a reduction of the stochastic shallow-water model to the CAM noise model as an alternative to the reduction in [Sura and Gille, 2010]. Then in section 2.3, we further analyse the properties of the CAM model for noise-driven SSH variability. In section 2.4, we develop a new statistical test for which parameters can be estimated from observed time series. A summary and discussion (section 2.5) concludes the chapter.

\subsection{An alternative motivation for the CAM model}

A model which has been traditionally used to study the propagation of surface waves in the ocean is a barotropic shallow-water model in a spherical sector basin with a flat bottom. It is well-known that such a model contains the whole spectrum of waves, e.g. Poincaré and Rossby waves as well as Kelvin waves along the boundaries. It should, therefore, serve as an elementary model to study wind-stress noise-induced variability in sea-level variations.

In [Sura and Gille, 2010], the CAM model for sea-level variability was motivated by considering small-scale nonlinear (advective) interactions in the shallowwater model. However, the precise interactions leading to the multiplicative noise component are unclear. Below, an alternative (or additional) motivation for the CAM noise model is considered just through the wind-stress variations.

\subsubsection{The stochastic shallow-water model}

Consider an ocean basin with a horizontal domain bounded by a closed contour $\Gamma$. The density of the ocean is constant and the flow is driven by a wind stress $\tau^{*}(\phi, \theta)=\tau_{0}\left(\tau^{\phi}, \tau^{\theta}\right)$, where $\tau_{0}$ is its amplitude and $\left(\tau^{\phi}, \tau^{\theta}\right)$ provides the spatial pattern. We denote $(u, v)$ as the horizontal velocities and $h$ as the depth of 
the basin. The governing shallow-water equations are non-dimensionalised using scales $r_{0}, H, U, r_{0} / U$, and $\tau_{0}$ for length, layer depth, velocity, time, and wind stress, respectively, where $r_{0}$ is the radius of the earth and become (for a flat-bottom ocean)

$$
\begin{gathered}
\epsilon\left(\frac{\partial u}{\partial t}+\frac{u}{\cos \theta} \frac{\partial u}{\partial \phi}+v \frac{\partial u}{\partial \theta}-u v \tan \theta\right)-v \sin \theta= \\
=-\frac{\epsilon F}{\cos \theta} \frac{\partial h}{\partial \phi}+E\left(\nabla^{2} u-\frac{u}{\cos ^{2} \theta}-\frac{2 \sin \theta}{\cos ^{2} \theta} \frac{\partial v}{\partial \phi}\right)+\alpha \frac{\tau^{\phi}}{h} \\
\epsilon\left(\frac{\partial v}{\partial t}+\frac{u}{\cos \theta} \frac{\partial v}{\partial \theta}+v \frac{\partial v}{\partial \theta}-u^{2} \tan \theta\right)+u \sin \theta= \\
=-\epsilon F \frac{\partial h}{\partial \theta}+E\left(\nabla^{2} v-\frac{v}{\cos ^{2} \theta}-\frac{2 \sin \theta}{\cos ^{2} \theta} \frac{\partial u}{\partial \phi}\right)+\alpha \frac{\tau^{\theta}}{h} \\
\frac{\partial h}{\partial t}+\frac{1}{\cos \theta}\left(\frac{\partial(h u)}{\partial \phi}+\frac{\partial(h v \cos \theta)}{\partial \theta}\right)=0
\end{gathered}
$$

where the dimensionless parameters are given by

$$
\begin{cases}\epsilon=\frac{U}{2 \Omega r_{0}} & \text { Rossby number } \\ F=\frac{g H}{U^{2}} & \text { Inverse Froude number } \\ E=\frac{A_{H}}{2 \Omega r_{0}^{2}} & \text { Ekman number } \\ \alpha=\frac{\tau_{0}}{2 \Omega \rho H U} & \text { Wind-stress coefficient }\end{cases}
$$

At the horizontal boundaries, no-slip boundary conditions are prescribed, i.e.,

$$
u(\phi, \theta)=v(\phi, \theta)=0 \quad \forall(\phi, \theta) \in \Gamma .
$$

The wind-stress forcing has a deterministic steady part $\left(\tau_{d}^{\phi}, \tau_{d}^{\theta}\right)$ and a stochastic part where the latter, following [Sura et al., 2001], can be prescribed in terms of atmospheric horizontal surface velocities $U_{a}$ and $V_{a}$ anomalies. Hence,

$$
\left(\tau^{\phi}, \tau^{\theta}\right)=\left(\tau_{d}^{\phi}, \tau_{d}^{\theta}\right)+\sigma\left(U_{a}, V_{a}\right)
$$

where $\sigma$ is the amplitude of the noise. In addition,

$$
\left(U_{a}, V_{a}\right)=z(\phi, \theta)\left(\eta_{u}(\phi, \theta, t), \eta_{v}(\phi, \theta, t)\right),
$$

where $z(\phi, \theta)$ is a given spatial dimensionless pattern and the quantities $\eta_{u}$ and $\eta_{v}$ represent uncorrelated (white) noise and satisfy

$$
\left\langle\eta_{u}\left(\phi_{1}, \theta_{1}, t\right), \eta_{u}\left(\phi_{2}, \theta_{2}, s\right)\right\rangle=\delta\left(\phi_{1}-\phi_{2}\right) \delta\left(\theta_{1}-\theta_{2}\right) \delta(t-s) .
$$

The same holds for $\left\langle\eta_{u}\left(\phi_{1}, \theta_{1}, t\right), \eta_{v}\left(\phi_{2}, \theta_{2}, s\right)\right\rangle$ and $\left\langle\eta_{v}\left(\phi_{1}, \theta_{1}, t\right), \eta_{v}\left(\phi_{2}, \theta_{2}, s\right)\right\rangle$. The weight function $z(\phi, \theta)$ can for example be a Gaussian shape with circular 
symmetry to the atmospheric variability [Sura et al., 2001]. As already discussed in the previous chapter, the white-noise structure of the atmospheric forcing is an idealization: it reflects the time scale separation between the slow 'climate system', represented by the ocean, and the fast 'weather system', represented by the atmosphere [Hasselmann, 1976].

\subsubsection{Linearised system}

We can write the solutions in the model as

$$
u=u_{d}+u^{\prime} ; v=v_{d}+v^{\prime} ; h=h_{d}+h^{\prime},
$$

where $\left(u_{d}, v_{d}, h_{d}\right)$ are the steady state velocities and thickness field for the deterministic wind-stress field. For example, when $\tau_{d}^{\phi}=\tau_{d}^{\theta}=0$, the equilibrium state is represented by the ocean at rest, with a reference height $h_{0}$, i.e. $\left(u_{0}, v_{0}, h_{0}\right)=$ $\left(0,0, h_{0}\right)$.

Under the assumption of linearised dynamics and a zero deterministic windstress field, eqs. (2.2.1) for the perturbations $\left(u^{\prime}, v^{\prime}, h^{\prime}\right)$ become

$$
\begin{gathered}
\epsilon \frac{\partial u^{\prime}}{\partial t}-v^{\prime} \sin \theta=-\frac{\epsilon F}{\cos \theta} \frac{\partial \eta^{\prime}}{\partial \phi}+E\left(\nabla^{2} u^{\prime}-\frac{u^{\prime}}{\cos ^{2} \theta}-\frac{2 \sin \theta}{\cos ^{2} \theta} \frac{\partial v^{\prime}}{\partial \phi}\right)+\left(\frac{1}{h_{0}}-\frac{1}{h_{0}^{2}} h^{\prime}\right) \alpha \tau^{\phi}, \\
\epsilon \frac{\partial v^{\prime}}{\partial t}+u^{\prime} \sin \theta=-\epsilon F \frac{\partial \eta^{\prime}}{\partial \theta}+E\left(\nabla^{2} v^{\prime}-\frac{v^{\prime}}{\cos ^{2} \theta}-\frac{2 \sin \theta}{\cos ^{2} \theta} \frac{\partial u^{\prime}}{\partial \phi}\right)+\left(\frac{1}{h_{0}}-\frac{1}{h_{0}^{2}} h^{\prime}\right) \alpha \tau^{\theta}, \\
\frac{\partial h^{\prime}}{\partial t}+\frac{h_{0}}{\cos \theta}\left(\frac{\partial u^{\prime}}{\partial \phi}+\frac{\partial\left(v^{\prime} \cos \theta\right)}{\partial \theta}\right)=0 .
\end{gathered}
$$

When we discretise the system on a two-dimensional grid in $(\phi, \theta)$, we can write it in the following matrix form

$$
\mathcal{M} \frac{\mathrm{d} \mathbf{X}}{\mathrm{d} t}=\mathcal{A} \mathbf{X}+(\mathcal{B}+\mathcal{D} \mathbf{X}) \boldsymbol{\eta},
$$

where $\mathbf{X} \in \mathbb{R}^{3 n}$ is the state vector, with $n$ representing the number of grid points, $\mathcal{A}, \mathcal{M}$ and $\mathcal{B}$ are $3 n \times 3 n$ matrices, $\mathcal{D}$ is a $3 n \times 3 n \times 3 n$ third order tensor and $\eta$ is a $3 n$-dimensional vector of uncorrelated white noise with unit intensity. The origin of the multiplicative noise term, involving the tensor $\mathcal{D}$, has a direct physical interpretation: the input of momentum by the wind stress depends on the thickness of the layer.

If we represent the state vector $\mathbf{X}$ of eq. (2.2.9) in the form $(\mathbf{u}, \mathbf{v}, \mathbf{h})$ (here we drop the primes to simplify the notation), and recalling that the matrix $\mathcal{M}$ is a diagonal matrix composed respectively of $\epsilon$ for the $\mathbf{u}$ and $\mathbf{v}$ part and 1 for the $\mathbf{h}$ part, we can rewrite the SDE (2.2.9) in the form

$$
\left\{\begin{array}{l}
\epsilon \frac{\mathrm{d} \mathbf{u}}{\mathrm{d} t}=\alpha_{u u} \mathbf{u}+\alpha_{u v} \mathbf{v}+\alpha_{u h} \mathbf{h}+\left(\beta_{h}+\delta_{h} \mathbf{h}\right) \boldsymbol{\eta}_{u}, \\
\epsilon \frac{\mathrm{d} \mathbf{v}}{\mathrm{d} t}=\alpha_{v u} \mathbf{u}+\alpha_{v v} \mathbf{v}+\alpha_{v h} \mathbf{h}+\left(\beta_{h}+\delta_{h} \mathbf{h}\right) \boldsymbol{\eta}_{v}, \\
\frac{d \mathbf{h}}{d t}=\alpha_{h u} \mathbf{u}+\alpha_{h v} \mathbf{v},
\end{array}\right.
$$


where each $\alpha_{i j}$ is a squared matrix (part of the matrix $\mathcal{A}$ ) $\beta_{h}$ and $\delta_{h}$ are the only non-zero components, respectively of the matrix $\mathcal{B}$ and the tensor $\mathcal{D}$. The quantities $\boldsymbol{\eta}_{u}$ and $\boldsymbol{\eta}_{v}$ are the two independent sub-vectors of the vector $\boldsymbol{\eta}$. The term $\delta_{h} \mathbf{h} \boldsymbol{\eta}_{u}$ has to be interpreted as $\left(\delta_{h}\right)_{i j k} h_{j}\left(\eta_{u}\right)_{k}$, with summation over repeated indices.

On the large scale, $\epsilon \ll 1$ while $\epsilon F=\mathcal{O}(1)$ and hence we can neglect the lefthand side in both the first two equations, and rewrite the vectors $\mathbf{u}$ and $\mathbf{v}$ in terms of $h$ and the noise (assuming invertibility of the block matrix formed by $\alpha_{u u}, \alpha_{u v}, \alpha_{v u}$ and $\alpha_{v v}$ ). Substituting these equations in the time evolution for $\mathbf{h}$, we have

$$
\frac{\mathrm{d} \mathbf{h}}{\mathrm{d} t}=\tilde{\alpha} \mathbf{h}+(\tilde{\beta}+\tilde{\delta} \mathbf{h}) \boldsymbol{\eta}_{h}
$$

with an appropriate definition of the parameters. Thanks to the independence of $\boldsymbol{\eta}_{u}$ and $\boldsymbol{\eta}_{v}$, it is guaranteed that $\boldsymbol{\eta}_{h}$ is also a white-noise term.

\subsection{Analysis}

As in the model of [Hasselmann, 1976], we now neglect the interaction between the sea-level height at different locations so that we only capture the local effects of the wind-stress noise (which are assumed to be the strongest). This is the principle of diagonal dominance of $\tilde{\alpha}$ in [Sura and Sardeshmukh, 2008], and hence we focus on the scalar version of (2.2.11), given by

$$
\frac{\mathrm{d} h}{\mathrm{~d} t}=\tilde{A} h+(\tilde{B}+\tilde{D} h) \eta_{h}
$$

where now $h, \tilde{A}, \tilde{B}$ and $\tilde{D}$ are scalars. From a physical point of view, the constants can be interpreted as the effect of the local near-geostrophic response on the sea level. The multiplicative noise term arises because the input of momentum by the wind stress depends on the total layer thickness. This is different from the SST model in [Hasselmann, 1976], where the atmospheric heat flux into the mixed layer does not depend on the total mixed layer depth. Note that a more extended mixed-layer model as in [Sura and Sardeshmukh, 2008], where also the dependence of the perturbative heat flux on SST is taken into account, does include a multiplicative noise term.

Eq. (2.3.1) captures the effects of the wind-driven noise on sea-level variability in its simplest way. However, without any indication of the appropriate choice of the interpretation of the noise term, we are not able to solve it. In fact, when we try to solve the equation, we have to decide whether to use the Itô or the Stratonovich calculus [Pavliotis, 2016]. In order to make this choice, we have to look at the origin of the noise in the system. As we pointed out in section 2.2.1, the white-noise structure of the wind forcing is the limit case of a fast decorrelating atmospheric variability. It can be shown [van Kampen, 1981] that these kind of systems can be approximated by equations with white noise using the Stratonovich 
interpretation. Therefore, a more correct way to write eq. (2.3.1) is the following one

$$
\mathrm{d} \tilde{h}_{t}=\tilde{A} \tilde{h}_{t} \mathrm{~d} t+\left(\tilde{B}+\tilde{D} \tilde{h}_{t}\right) \circ \mathrm{d} W_{t},
$$

where the symbol $\circ$ is conventionally chosen to represent a Stratonovich SDE and the subscript $t$ is referring to the time dependence of the stochastic process. However, from a mathematical point of view, it is usually easier to deal with Itô SDEs, especially because they constitute Markovian processes, in contrast with Stratonovich SDEs.

It is easy to show [Pavliotis, 2016] that eq. (2.3.2) is equivalent to the Itô SDE

$$
\mathrm{d} h_{t}=A h_{t} \mathrm{~d} t+\left(B+D h_{t}\right) \mathrm{d} W_{t},
$$

where

$$
h_{t}=\frac{2 \tilde{A}+\tilde{D}^{2}}{\tilde{B} \tilde{D}} \tilde{h}_{t}+1 \text {, }
$$

and

$$
A=\tilde{A}+\frac{1}{2} \tilde{D}^{2} ; B=\frac{2 \tilde{A}}{\tilde{D}} ; D=\tilde{D} .
$$

\subsubsection{Probability density function}

Consider now eq. (2.3.3) with $A<0$ and $B, D>0$. The corresponding FokkerPlanck equation is

$$
\frac{\partial p}{\partial t}=-\frac{\partial(A h p)}{\partial h}+\frac{1}{2} \frac{\partial^{2}\left[(B+D h)^{2} p\right]}{\partial h^{2}},
$$

where the solution $p=p(h, t)$ is the probability density function of the stochastic variable $h_{t}$ at time $t$. Furthermore, the boundary conditions for the distribution and its derivative in space are

$$
p, \frac{\partial p}{\partial h} \rightarrow 0 \text { for } h \rightarrow \pm \infty .
$$

We are interested in the stationary distribution, that is

$$
p_{s}(h):=\lim _{t \rightarrow \infty} p(h, t) .
$$

Hence eq. (2.3.4) becomes

$$
A h p_{s}-\frac{1}{2} \frac{\mathrm{d}\left[(B+D h)^{2} p_{s}\right]}{\mathrm{d} h}=0,
$$

which can be solved to give

$$
p_{s}(h)=k \exp \left\{\frac{2 A}{D^{2}}\left[\left(1-\frac{D^{2}}{A}\right) \ln |D h+B|+\frac{B}{D h+B}\right]\right\},
$$


with $k$ normalization constant. In case $D$ is positive, we can calculate the one-sided limits close to $x=-B / D$ as

$$
\lim _{h \rightarrow-\frac{B}{D}^{-}} p_{s}(h)=e^{\infty}=\infty,
$$

and

$$
\lim _{h \rightarrow-\frac{B}{D}^{+}} p_{s}(h)=e^{-\infty}=0,
$$

and hence the integral of $p_{s}(h)$ over the whole domain diverges. By definition, a solution of the Fokker Planck equation has to be smooth and integrable over the domain [Pavliotis, 2016]. Hence for $D>0$, the solution is given by

$$
p_{s}(h)= \begin{cases}0 & h \leq-B / D \\ N \exp \left\{\frac{2 A}{D^{2}}\left[\left(1-\frac{D^{2}}{A}\right) \ln |D h+B|+\frac{B}{D h+B}\right]\right\} & h>-B / D\end{cases}
$$

where $N$ is the normalization constant. One can show that $p_{s}(h)$ satisfies the stationary Fokker-Planck equation and the requirements for its solution. In case $D<0$, the solution is found as

$$
p_{s}(h)= \begin{cases}N \exp \left\{\frac{2 A}{D^{2}}\left[\left(1-\frac{D^{2}}{A}\right) \ln |D h+B|+\frac{B}{D h+B}\right]\right\} & h \leq-B / D \\ 0 & h>-B / D\end{cases}
$$

In Fig. 2.1(a), a series of trajectories is seen to converge to an equilibrium distribution and the histogram in Fig. 2.1(b) can be well represented by the analytically determined $p_{s}$.

\subsubsection{Moments of the distribution}

It is useful to calculate the moments of the distribution. In principle we could determine the moments of the stationary distribution, starting from the distribution itself

$$
M_{n}:=\int p_{s}(h) h^{n} \mathrm{~d} h .
$$

The integrals cannot be solved analytically but ODEs for the moments can be obtained using an Itô formula. Given a one dimensional SDE

$$
\mathrm{d} h_{t}=\theta\left(h_{t}\right) \mathrm{d} t+\sigma\left(h_{t}\right) \mathrm{d} W_{t}
$$

and a smooth function $f\left(h_{t}\right): \mathbb{R}^{n} \rightarrow \mathbb{R}$, the following (Itô) formula holds [Pavliotis, 2016]

$$
\mathrm{d} f\left(h_{t}\right)=\left(\theta\left(h_{t}\right) f^{\prime}\left(h_{t}\right)+\frac{\sigma^{2}\left(h_{t}\right)}{2} f^{\prime \prime}\left(h_{t}\right)\right) \mathrm{d} t+\sigma\left(h_{t}\right) f^{\prime}\left(h_{t}\right) \mathrm{d} W_{t} .
$$


(a)

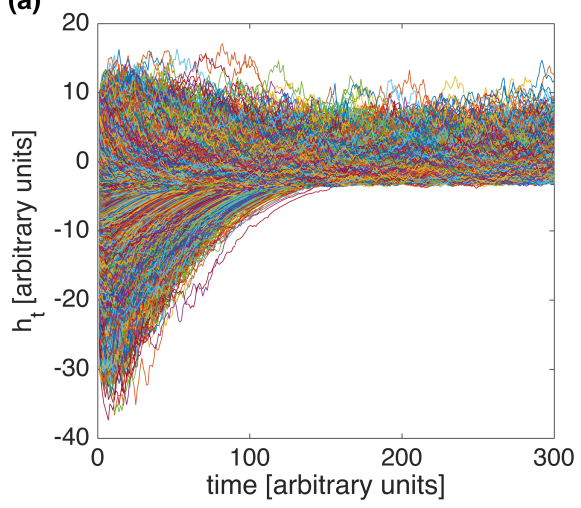

(b)

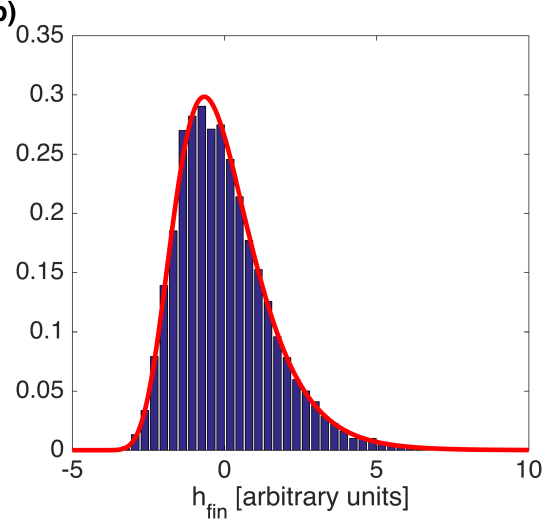

Figure 2.1: (a) Trajectories of the system (2.3.3), with parameters $(A, B, D)=$ $(-2.00,3.00,0.50)$, initiated from 100 points uniformly distributed in the interval $[-30,10]$ : it is clear that, after a certain amount of time, all the solutions remain above a certain threshold. (b) Histogram relative to the final distribution of the end points (after $10^{5}$ time steps) of the trajectories in (a). On top of it, the PDF (2.3.11), drawn with a red line, perfectly fits the histogram.

Defining the $n$-th moment as

$$
M_{n}(t)=\mathbf{E}\left[h^{n}(t)\right],
$$

we can write an evolution equation for $M_{n}$ by combining eq. (2.3.3) and eq. (2.3.15), resulting in

$$
\frac{\mathrm{d} M_{n}}{\mathrm{~d} t}=n\left(A+\frac{n-1}{2} D^{2}\right) M_{n}+n(n-1) B D M_{n-1}+\frac{n(n-1)}{2} B^{2} M_{n-2} .
$$

For the first four moments, we obtain

$$
\left\{\begin{array}{l}
\frac{\mathrm{d} M_{1}}{\mathrm{~d} t}=A M_{1} \\
\frac{\mathrm{d} M_{2}}{\mathrm{~d} t}=2\left(A+\frac{D^{2}}{2}\right) M_{2}+2 B D M_{1}+B^{2} \\
\frac{\mathrm{d} M_{3}}{\mathrm{~d} t}=3\left(A+D^{2}\right) M_{3}+6 B D M_{2}+3 B^{2} M_{1} \\
\frac{\mathrm{d} M_{4}}{\mathrm{~d} t}=4\left(A+\frac{3 D^{2}}{2}\right) M_{4}+12 B D M_{3}+6 B^{2} M_{2}
\end{array}\right.
$$

At equilibrium (corresponding to the stationary distribution) we can explicitly calculate the moments in terms of the constants $A, B$ and $D$ as

$$
\left\{\begin{array}{l}
M_{1}=0 \\
M_{2}=-\frac{B^{2}}{2 A+D^{2}} \\
M_{3}=\frac{2 B^{3} D}{\left(A+D^{2}\right)\left(2 A+D^{2}\right)} \\
M_{4}=\frac{3 B^{4}\left(A-3 D^{2}\right)}{\left(2 A+D^{2}\right)\left(A+D^{2}\right)\left(2 A+3 D^{2}\right)}
\end{array}\right.
$$


which corresponds (with a different notation) to those obtained in [Sura and Sardeshmukh, 2008]. Hence we can compute some interesting statistical quantities relative to CAM noise (mean $\mu$, variance $C$, skewness $S$ and kurtosis $K$ ):

$$
\left\{\begin{array}{l}
\mu=0 \\
C=-\frac{B^{2}}{2 A+D^{2}} \\
S=-\frac{2 D\left(-\left(2 A+D^{2}\right)\right)^{1 / 2}}{A+D^{2}} \\
K=\frac{3\left(A-3 D^{2}\right)\left(2 A+D^{2}\right)}{\left(A+D^{2}\right)\left(2 A+3 D^{2}\right)}
\end{array}\right.
$$

As expected, the statistical properties of the solution $h_{t}$ are quite different from the ones obtained from an Ornstein-Uhlenbeck process. For instance, the distribution is asymmetric (skewness different from zero) and the kurtosis (different from three) indicates a heavy-tail behaviour. It is easy to show that, for $D=0$, we can recover the corresponding moments for the Ornstein-Uhlenbeck process. Furthermore, in order for the variance to be positive, we have to impose the condition $2 A+D^{2}<0$ for the SDE (2.3.3).

\subsubsection{Temporal discretization}

The easiest way to perform a temporal discretization of the SDE (2.3.3) is using the Euler-Maruyama scheme [Gardiner et al., 1985], resulting in

$$
h_{t}=(1+A \Delta t) h_{t-1}+\left(B+D h_{t-1}\right) \sqrt{\Delta t} \eta_{t},
$$

with $\Delta t$ the time step and $\eta_{t}$ time uncorrelated white noise, with zero-mean unitary variance. The stochastic process (2.3.21) is equivalent to eq. (2.3.3), assuming that $\Delta t$ is small. The same result is valid in the comparison between an AR(1) and an Ornstein-Uhlenback process. A simple argument to prove this statement can be given calculating the second and the third moment of the discrete process (2.3.21) and expressing them in terms of the parameters $A, B, D$ and $\Delta t$. In the limit $\Delta t \rightarrow 0$, we can neglect the higher order terms and the equations turn out to be equivalent to the moments of a SDE with CAM noise (2.3.19).

Suppose we have a time series of SSH data and want to determine whether the stochastic process (2.3.21) can describe the statistics of the time series, how do we estimate the parameters in this equation? While the literature concerning the estimation of the parameters of an Ornstein-Uhlenbeck process is quite broad, to our knowledge there is no systematic approach existing for the process (2.3.21). The first thing to notice is that we are not able to perform the estimation basing it only on the stationary distribution of the corresponding SDE, or on its related statistical quantities. In fact, one can always rescale the parameters of the continuous CAM process, and also rescale the time. In other words, the distribution is invariant under the following transformation

$$
A^{\prime}=k A \quad B^{\prime}=\sqrt{k} B \quad D^{\prime}=\sqrt{k} D,
$$

with $k>0$. 


\subsubsection{Parameter estimation}

As for the red noise case, in order to make the estimation unique, it is necessary to include some information about the temporal behaviour of the time series in the procedure. For instance, we could use the autocorrelation structure of the time series. To this effect, we first have to determine the autocorrelation function for a SDE with CAM noise (2.3.3). Using the general solution of the SDE [Pavliotis, 2016]

$$
\begin{gathered}
h_{t}=y_{t}\left(X_{0}-B D \int_{0}^{t} y_{s}^{-1} \mathrm{~d} s+B \int_{0}^{t} y_{s}^{-1} \mathrm{~d} W_{s}\right), \\
y_{t}=\exp \left[\left(A-\frac{1}{2} D^{2}\right) t+D W_{t}\right]
\end{gathered}
$$

one can show that

$$
\tilde{\gamma}(s)=\lim _{t \rightarrow \infty}\left\langle h_{t} h_{t+s}\right\rangle=-\frac{B^{2}}{2 A+D^{2}} \exp (A s) .
$$

Knowing that the term in front of the exponential function is the variance of the SDE, we can rewrite the normalised autocorrelation function for the corresponding stochastic process as

$$
\gamma(n \Delta t)=\frac{\tilde{\gamma}(n \Delta t)}{M_{2}}=\exp (A \Delta t n)
$$

where $n$ represents the time lag. Through the last equation, substituting the autocorrelation function with its estimated value, and choosing the value of $\Delta t$ (providing that we use the same value in the simulation of the process), it is possible to determine the parameter $A$. In order to estimate the other two parameters, we are going to use two different methods: a moments estimation and a fit of the data with the probability density function.

One of the most well-established methods to estimate the parameters of an Ornstein-Uhlenbeck process consists in writing the autocorrelation function and the second moment (that is the variance, for a zero-mean process) in terms of the parameters, then estimating these two quantities from data and substituting the values in the equations. In our case, since we have an additional parameter (compared to the Ornstein-Uhlenbeck process), we need another equation, namely the third moment. Therefore, after having found $A$ with the autocorrelation function, we are going to make use of the moment equations

$$
\hat{M}_{2}=-\frac{B^{2}}{2 A+D^{2}}, \quad \hat{M}_{3}=\frac{2 B^{3} D}{\left(A+D^{2}\right)\left(2 A+D^{2}\right)},
$$

where the quantities $\hat{M}_{2}$ and $\hat{M}_{3}$ are respectively the estimated second and third moment. In Fig. 2.2(a) the result of the estimation for a simulated time series is shown (see the figure caption for further details), and it is shown to be successful. 
(a)

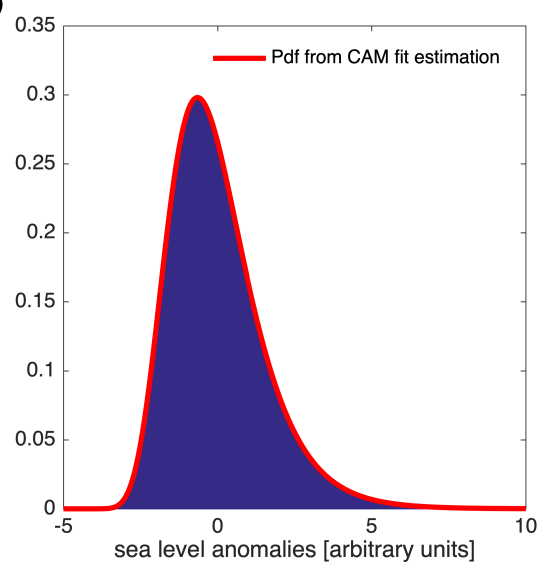

(b)

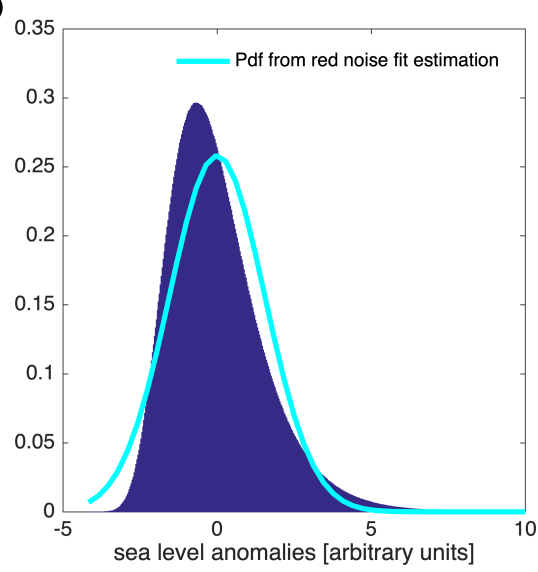

Figure 2.2: (a) Normalised histogram obtained from a long simulated time series, from a CAM noise SDE. The parameters A,B and D are $(-2.00,3.00,0.50)$. The estimated parameters with the moments method for a CAM process are $(-2.00,2.99,0.49)$. The red line is the analytical pdf (2.3.11), with the estimated parameters. (b) Normalised histogram obtained from a long simulated time series, from a CAM noise SDE. The parameters are $(-2.00,3.00,0.50)$. The estimated parameters with the moments method for a red noise process are $(-2.00,3.09)$. The cyan line is the analytical Gaussian pdf for the estimated parameters. 
The same data can be used to estimate the parameters $A$ and $B$ via the autocorrelation function and the second moment of the Ornstein-Uhlenbeck process, and the result is shown in Fig. 2.2(b). It is clear that the estimated parameters differ substantially from the true ones. The result is a distribution which doesn't resemble at all the true one. The reason behind this behaviour has to be found in the statistical properties of the stochastic processes under consideration: while the Gaussian distribution is completely determined by its first two moments, this is not the case for more complex processes, such as the SDE with CAM noise (2.3.3). Therefore, even if the autocorrelation function and the variance of two processes are the same, it doesn't mean they have the same distribution, assuming that at least one of them is not Gaussian.

Therefore, in order to make the test of the null hypothesis meaningful, one should consider, together with the power spectrum analysis, an evaluation of the accuracy of the parameters estimation. To this effect, we introduce a $\chi$-square measure

$$
\chi=\frac{1}{L} \sum_{i=1}^{L}\left(F\left(z_{i}\right)-p_{s}\left(z_{i}\right)\right)^{2},
$$

where $z_{i}$ is the midpoint of the $i$-th interval obtained by binning the data ( $L$ being the number of bins), $F\left(z_{i}\right)$ is the corresponding value of the normalised histogram, and $p_{s}(x)$ is the stationary probability density function under consideration (depending on which model is used for the estimation). The $\chi$ measure represents the distance between the distribution of the data and the one predicted by the model. It has to be noticed that this measure is very useful in order to evaluate which distribution is the closest one to the one obtained from the data. Nevertheless, it can't be used to compare the accuracy of a certain estimation for different time series: its value, indeed, depends on how much the data is spread around the mean, assuming a constant $k$. For the simulated time series in Fig. 2.2, we obtain $\chi_{\mathrm{RED}}^{\text {sim }} \sim 10^{-4}, \chi_{\mathrm{CAM}}^{\text {sim }} \sim 10^{-7}$, where the subscript $R E D$ refers to red noise, i.e., Ornstein-Uhlenbeck process with a Gaussian distribution. As expected, the comparison between the two measures allows us to state that the CAM estimation works well, whereas the Ornstein-Uhlenbeck process is not an accurate model for our simulated data.

Another method of estimation consists in fitting the histogram of the time series under consideration with a certain distribution (which in the CAM case is represented by eq. (2.3.11)). That means solving the following non-linear least-squares problem (for unknowns $A, B$ and $D$ )

$$
\min \sum_{i=1}^{L}\left|F\left(z_{i}\right)-p_{s}\left(A, B, D, z_{i}\right)\right|^{2},
$$

where $z_{i}$ is the midpoint of the $i$-th interval obtained by binning the data ( $L$ being again the total number of bins), $F\left(z_{i}\right)$ is the corresponding value of the normalised histogram, and $p_{s}(A, B, D, z)$ is the stationary probability density function for the 


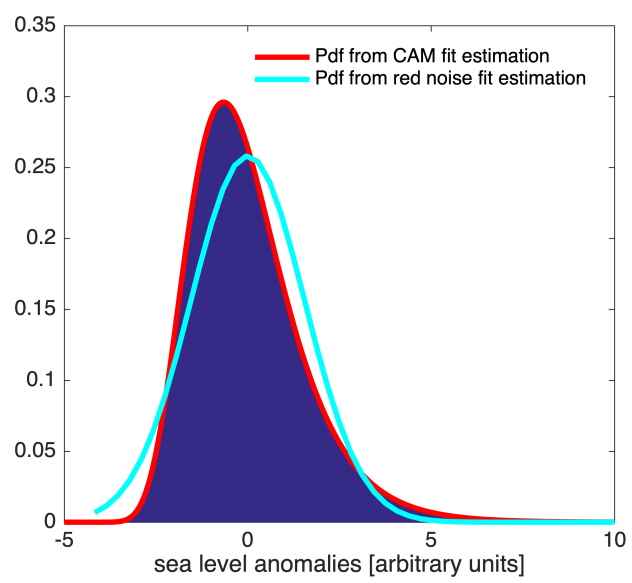

Figure 2.3: Normalised histogram obtained from a long simulated time series, from a CAM noise SDE. The parameters are $(-2.00,3.00,0.50)$. The estimated parameters with the fit method for a CAM process are $(-2.00,3.01,0.49)$, whereas for a red noise process are $(-2.00,3.09)$. The red and cyan lines are the analytical pdf, respectively CAM and red noise, with the estimated parameters.

chosen model. In order to perform the least-squares problem, we used the MATLAB function lsqnonlin, which computes $L$ functions and finds the minimum of the norm (2.3.28). The choice of the initial condition seems to be crucial for the convergence of the algorithm, especially for a high number of functions: in our code we performed the optimisation starting from the computation of only 2 functions (corresponding to a fit with a histogram with 2 bins), choosing a certain initial condition. Later, we incremented the number of bins and chose, as initial condition, the parameters found in the previous step. Then we iterated the procedure until the maximum number of bins (100) was reached.

In Fig. 2.3 the result of the estimation for the simulated time series under consideration is shown (see the figure caption for further details). Again we can compute the $\chi$ measures for the two estimates, which turn out to be very similar to the previous ones, i.e., $\chi_{\mathrm{RED}}^{\text {sim }} \sim 10^{-4}, \chi_{\mathrm{CAM}}^{\text {sim }} \sim 10^{-7}$. For long simulated time series, at least for the CAM noise case, the two methods (moments and fit) give similar results; this is not the case for short ones, with possible consequences in the test of the null hypothesis. We consider the distribution fitting method the most effective one to estimate the parameters: with this method, indeed, we take into account also the other moments, while with the moment estimation we are only able to consider the second moment for a red noise process, and the second and third moment for a CAM noise process, respectively. 


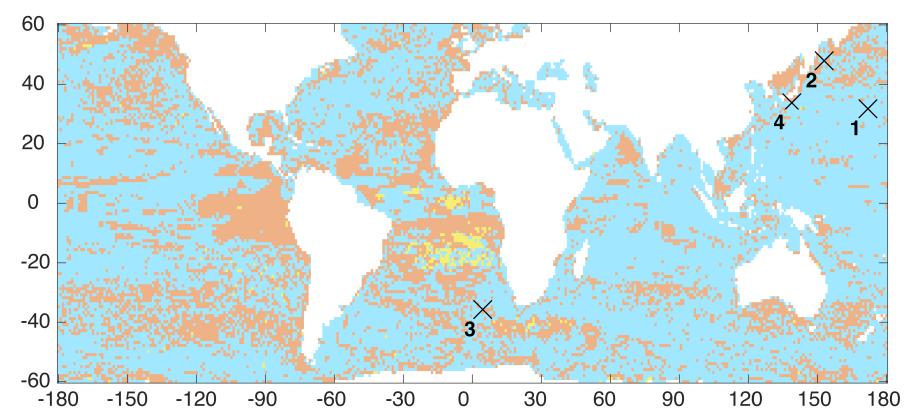

CAM estimation RED estimation Failed estimation

Figure 2.4: Ratio between $\chi$-squared measures calculated respectively from estimation with red and CAM noise. If the ratio is greater than one, then $\chi_{\mathrm{RED}}>\chi_{\mathrm{CAM}}$, which indicates that the CAM estimation works better: the locations showing this behaviour are coloured in red. If the ratio is less than one, then the red noise estimation works better and the correspondent location is coloured in cyan. Yellow-coloured locations correspond to distributions which do not resemble any of the two considered processes, hence both estimations fail $\left(\chi_{\mathrm{RED}}, \chi_{\mathrm{CAM}}>>1\right)$. The black crosses indicate the four locations for which more details are provided in the text. The resolution of the map is $1 \times 1$ degree.

\subsection{Application: Sea-level variability}

The distribution fitting method of the previous section is applied to SSH anomaly data in this section. The dataset we use consists in daily gridded multi-mission sea surface height anomalies, measured over about 20 years, collected by the Archiving, Validation and Interpretation of Satellite Oceanographic data base (AVISO, http://www.aviso.altimetry.fr/en/data.html).

In Fig. 2.4 we first give a representation of the accuracy of the CAM noise estimation with respect to the red noise one. For every location in the ocean, we performed the two estimations and compared the values of the respective values of the $\chi$-squared measure (see figure caption for further explanation). It is clear that the CAM estimation works (red colour) better in many locations of the oceans. It is also interesting to compare this result with the analysis of the skewness of the SSH distributions obtained from data, as determined in [Sura and Gille, 2010]. From the comparison between Fig. 2.4 and Fig. 3 in [Sura and Gille, 2010], we see that, when the distribution of data is skewed, the CAM estimation works well, especially in the cases where the skewness is positive.

From the results of section 2.3, we can now formulate the null hypothesis for the sea-level variability. Wind-stress (white) noise causes sea-level variability according to the CAM noise process and, if sea-level variability is attributed to different causes, the null hypothesis should be rejected. In order to test this hypothesis, the following procedure is used, as applied to sea surface anomaly data (mean zero, detrended, seasonal cycle removed): (i) estimate the parameters $A, B$ and $D$ in the CAM noise process, (ii) run many realizations of the stochastic process 
with the same time step as that in the data and (iii) determine power spectra for each realization. After choosing a significance level $\tilde{p}$, plot the $1-\tilde{p}$ quantile of the sampled spectra, for each frequency, together with the power spectrum of the data. If there are peaks extending above the chosen spectrum, it means that the null hypothesis is rejected at a $100(1-\tilde{p}) \%$ significance level. We applied this procedure for several time series, corresponding to different locations of the ocean (see crosses in Fig. 2.4), which represent the most interesting cases.

We first consider a location (location 1) where, according to Fig. 2.4, the sealevel statistics are approximately Gaussian. In Fig. 2.5, the results for the estimations and the power spectrum analysis are shown. The values of $\chi$ for both estimations are $\chi_{\mathrm{RED}}^{\text {sim }}=0.48, \chi_{\mathrm{CAM}}^{\text {sim }}=0.5$ and hence indeed the red noise estimation performs slightly better than the CAM process one. It has to be noticed that, when the distribution of the data is approximately Gaussian, the CAM process which solves eq. (2.3.28) will have a very small (positive) value of the parameter $D$. This is the reason why the difference between the two estimations, and the correspondent $\chi$ measures, is small. The situation is well represented in Fig. 2.5, where the curves relative respectively to red and CAM noise almost overlap, both in regard to the distribution and the power spectrum. The peaks extending above the CAM power spectrum ( 100-days period) are generated by the mesoscale eddy field [Holland, 1978] in this region.

Next, we look at a region where the distribution of the sea-level anomalies is definitely skewed. In Fig. 2.6, we present the results for a location in the North Pacific Ocean (location 2). The values of $\chi$ are $\chi_{\mathrm{RED}}^{\text {sim }}=0.91, \chi_{\mathrm{CAM}}^{\text {sim }}=0.14$, clearly showing that a CAM noise spectrum is much better than a red noise spectrum: Fig. 2.6(b) shows that some peaks, which seem to be significant under the red noise test are not significant under the CAM noise test. The only remaining significant peak is again generated by the mesoscale eddy field in that region.

As a third example, we consider the region west of South Africa (location 3). The Agulhas Current affects the South-West Indian Ocean, along the East coast of South Africa and, where it turns back on itself, it periodically releases an eddy into the South Atlantic Gyre. By investigating the variability of sea level in the area around the east coast of South Africa, we expect to observe a peak in the power spectrum, with a period of about 50 days [Beal et al., 2011]. Furthermore, the CAM noise model should not be able to represent the dynamics of the sea level, at least in the region of the spectrum where these phenomena occur (Fig. 2.7). The values of the $\chi$ measure are $\chi_{\mathrm{RED}}^{\text {sim }}=0.17, \chi_{\mathrm{CAM}}^{\text {sim }}=0.07$. Again, the ratio between the two measures is low, indicating that the two estimations give similar results: the distribution of the data, indeed, doesn't differ much from a Gaussian distribution. The 50-day peak is significant under both the red noise and CAM noise test.

A final case is a location where both the estimations fail (location 4). Observations over the last century indicate that Kuroshio current in the North Pacific Ocean shows bimodal behaviour off the southern coast of Japan [Qiu and Miao, 2000]. While the mechanisms leading to such bimodality have not been completely understood, it is clear that they must be part of the internal dynamics of the ocean and 
(a)

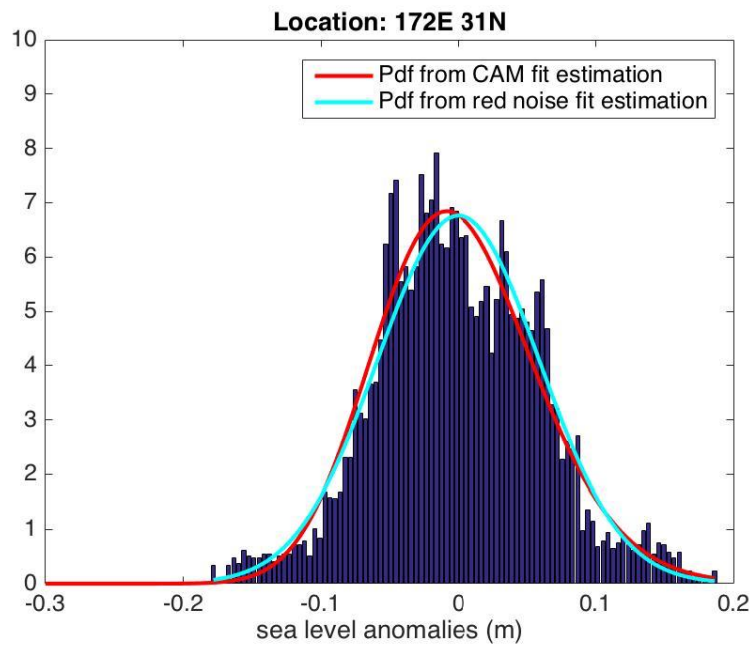

(b)

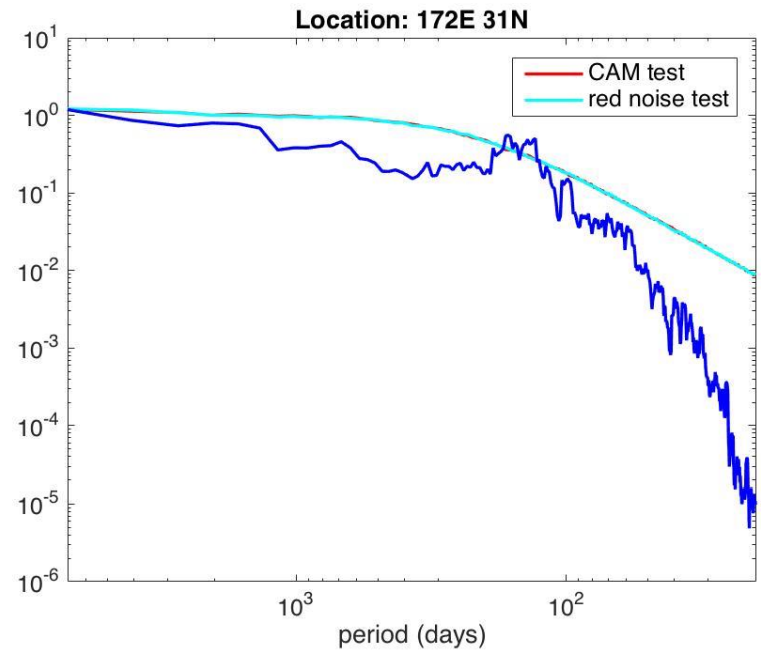

Figure 2.5: (a) Normalised histogram obtained from the time series relative to sea-level anomalies at the indicated location 1 . The red and cyan lines are the analytical pdf with the estimated parameters, respectively for CAM noise $(-0.03,0.01,0.02)$ and red noise $(-0.03,0.01)$. The two estimated distributions practically overlap, reflecting the almostGaussian shape of the histogram. See text for further explanations. (b) Null hypothesis test for the time series relative to sea-level anomalies in the indicated location. The blue line is the power spectrum of the data. The red and cyan lines represent the values of the power spectra relative to an ensemble of simulations, at the 0.01 level of significance for each frequency, respectively for CAM noise and red noise SDEs, with the estimated parameters. 
(a)

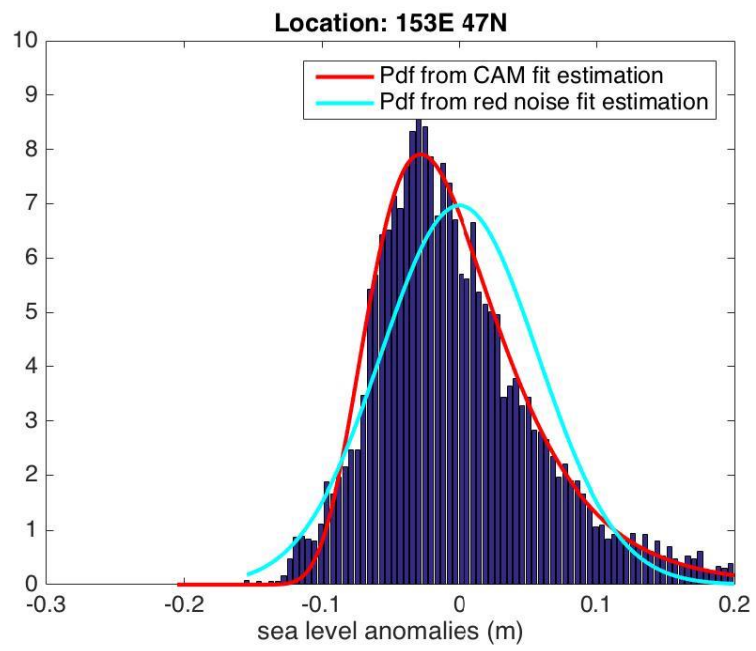

(b)

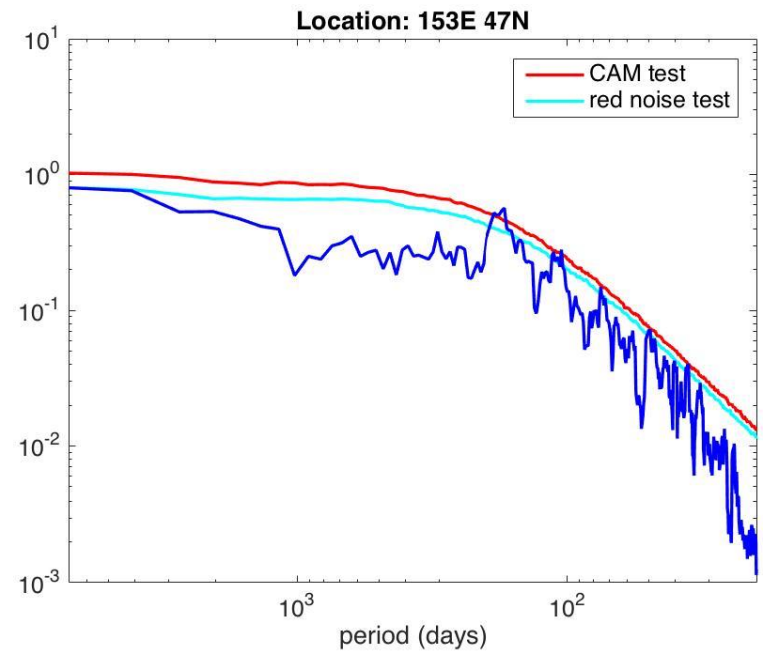

Figure 2.6: Same as Fig. 2.5, but for location 2. (a) The estimated parameters are respectively $(-0.04,0.02,0.08)$ for CAM noise and $(-0.04,0.02)$ for red noise. The two estimated distributions differ substantially, as indicated also by the value of the parameter $D$. (b) Many peaks, which are significant under the red noise test, are not significant under the CAM noise test. 
(a)

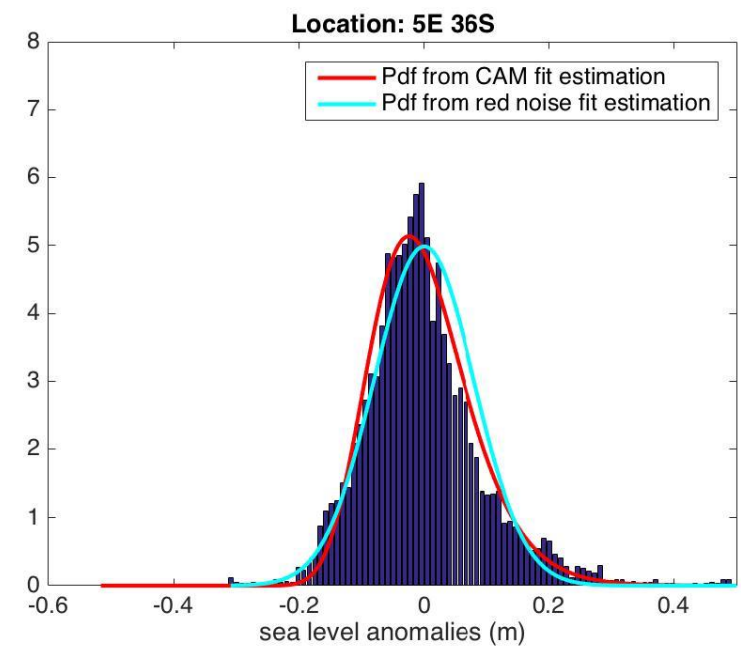

(b)

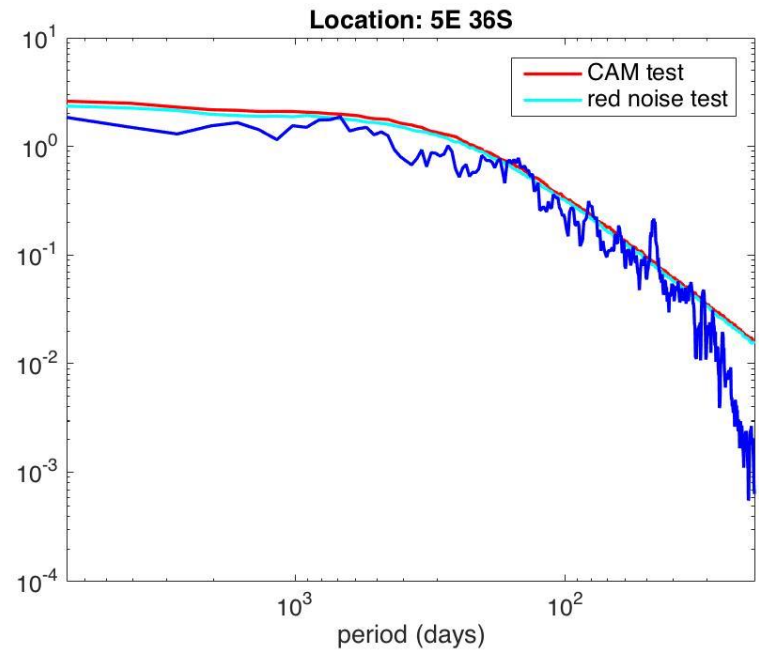

Figure 2.7: Same as Fig. 2.5, but for location 3. (a) The estimated parameters are respectively $(-0.03,0.02,0.04)$ for CAM noise and $(-0.03,0.02)$ for red noise. The two estimated distributions partially differ, as indicated also by the value of the parameter $D$. (b) The 50-days peak is significant under both the tests. 


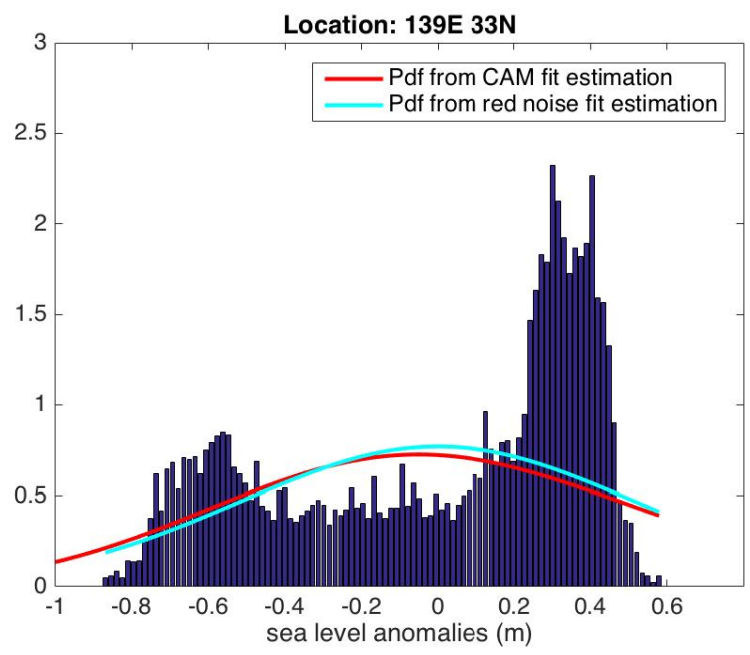

Figure 2.8: Normalised histogram obtained from the time series relative to sea-level anomalies at the indicated location 4 . The red and cyan lines are the analytical pdf with the estimated parameters, respectively for CAM noise $(-0.01,0.08,0.01)$ and red noise $(-0.01,0.07)$. It is clear how the estimated distributions differ from the distribution of the data. See text for further explanations.

thus they cannot be explained by wind-stress noise. In Fig. 2.8, the result of the estimation of the parameters is shown for one of these time series. The estimation has been performed for both an Ornstein-Uhlenbeck process and a CAM noise SDE. Even without investigating the power spectrum of the time series, we can conclude that our null-hypothesis has to be rejected: this example clarifies the importance of the accuracy of the estimations, as a preliminary investigation before proceeding with the null hypothesis test.

\subsection{Summary and discussion}

In this chapter, we formulated a statistical test associated with the null hypothesis that sea-level variability is only forced by random (white noise) fluctuations in the wind stress. In many studies on sea-level variability, one wants to distinguish the effects of specific flow phenomena, such as eddies, on sea-level variability from the noisy wind-stress driven variability. In [Sura and Gille, 2010], it was already argued that the basic SSH model (represented by the stochastic process $h_{t}$ representing sea-level anomalies) is given by the Itô SDE

$$
\mathrm{d} h_{t}=A h_{t} \mathrm{~d} t+\left(B+D h_{t}\right) \mathrm{d} W_{t},
$$


with parameters $A, B$ and $D$ and constraint $2 A+D^{2}<0$ (which implies that $A<0$ ). It was shown here that the choice of a stochastic linear model with CAM noise for the SSH variability also arises naturally from the shallow water equations under a random wind forcing, when only the local geostrophic flow effect due to the windstress forcing is considered. The multiplicative noise term arises because the momentum input by the wind is inversely proportional to the layer depth which makes the situation more complicated than in the classical Hasselmann model [Hasselmann, 1976] which captures only additive noise processes.

Whereas the estimation of parameters in the red noise process is relatively straightforward, the non-Gaussian behaviour of the SSH statistics requires a more sophisticated method of estimation (i.e. distribution fitting) with respect to the usual autocorrelation and moments evaluation. The reason is the fact that the knowledge of a few moments does not necessarily provide reliable information about the statistical distribution of the data, especially in the case the length of the time series is limited. Furthermore, we defined a $\chi$-squared measure, which is an indicator of the accuracy of our estimation of the PDF. For similar reasons, and relevant to the null hypothesis test, we argued that the power spectrum analysis is not enough: a preliminary study of the distribution is necessary to guarantee that the probability density function of the chosen stochastic process (with estimated parameters) can resemble the distribution of data. In other words, the evaluation of the accuracy of the estimation tells us whether the estimation with the chosen model is meaningful or not; if yes, then we can proceed with the power spectrum analysis.

The comparison between the $\chi$ measures for the different estimations (red and CAM) indicate that, in many locations of the ocean, the stochastic model with red noise is a good approximation. By contrast, for clearly skewed distributions, the CAM model is much more reliable than the red noise one. The comparison between the power spectra tells us that the CAM power spectrum extends always above the red noise one, and the distance between the two depends on the non-Gaussianity of the data.

Once the null-hypothesis was chosen, we performed a statistical test to reject it based on SSH data. From the power spectrum analysis of several selected time series, we can conclude that the CAM noise process under investigation can explain most of the variability of the sea level. When there are some peaks extending above the chosen $99 \%$ interval of confidence, they are attributable to physical processes different from wind-stress noise. In the Agulhas case, we clearly see a peak corresponding to a period of about 50 days, extending above the spectrum of the CAM process, as expected. Despite the fact that differences with red noise may be small in practice, the CAM noise test is in principle a better test and is also more stringent to attribute sea-level variability to specific phenomena. Although in the Agulhas case considered, this may not matter much, it can certainly matter in more extreme sea-level fluctuations as CAM has a power law distribution and the red noise an exponential one.

It has to be noticed that the null hypothesis test performed in this study has 
the desired significance level only in the case it is carried out for each single frequency. If one is interested in multiple testing, for instance when the same process is responsible for multiple peaks in the power spectrum, then a higher frequency pointwise confidence level has to be used [see section 5.2.5.1 in Mudelsee, 2013]. The consequence of not employing the correct multiple testing confidence interval is to detect false positives in the significant peaks.

Obviously, wind-stress noise is not the only source of noise induced by smallscale processes as is the case for SST, where noise is not only due to that in the atmospheric heat flux [Sura et al., 2006] and hence may also exhibit non-Gaussian behaviour. For the SSH case, also the heat flux is likely to be important through steric influences, as are small-scale processes in ocean mixing, such as sub-mesoscale processes. This defines a possible future line of study, where more and more complicated stochastic models can serve to define null hypotheses for small-scale induced variability in relevant observables in the climate system. 


\section{Chapter 3}

\section{Model and methods for transition probabilities}

This chapter is dedicated to introducing some of the concepts that are necessary for the following chapters. First of all, a box model for the AMOC is described in detail, and its suitability for addressing the second research question in section 1.4 is discussed. Second, a class of methods used to study rare events is introduced, with a particular focus on the Trajectory-Adaptive Multilevel Splitting (TAMS) algorithm. This method is the one chosen to calculate AMOC transition probabilities in the next chapters.

Part of this chapter can be found in the following publication (including its supplementary material):

Castellana, D., Baars, S., Wubs, F. W., and Dijkstra, H. A. (2019). Transition probabilities of noise-induced transitions of the Atlantic ocean Circulation, Scientific Reports, 9(1), 1-7.

The part relative to the rare event algorithms is adapted from the following manuscript: Wang, P., Castellana, D. and Dijkstra, H. A. (2020). Improvements to the use of the Trajectory-Adaptive Multilevel Sampling algorithm for the study of rare events. Manuscript under review. 


\subsection{A box model for the Atlantic Meridional Ocean Circulation}

In order to compute transition probabilities for a noise-induced collapse of the AMOC, we need to find a model that represents a compromise between two aspects. On one hand, it needs to be simple enough, so that it is computationally feasible to calculate transition probabilities with a rare event algorithm (introduced in the next section). On the other hand, as we want to compare our result with simulations from GCMs, the model needs to distinguish between the freshwater transport by the overturning circulation and the one carried by the subtropical gyre at the southern border of the Atlantic Ocean: therefore, Stommel's conceptual model is too simplistic to this purpose.

\subsubsection{Model formulation}

The model chosen is an improvement of the one developed by Cimatoribus et al. [2014], which was extended to allow the existence of a collapsed state of the AMOC. The Atlantic Ocean is represented as five boxes (Fig. 3.1). A deep box (labelled as $d$ ) extends throughout the whole latitudinal width of the basin. It is separated from the upper ocean layer by a pycnocline, whose depth $D$ is a dependent quantity in the model. Two other boxes $s$ and $n$ represent, respectively, the Southern Ocean and the northern part of the Atlantic Ocean, where the downwelling of dense water takes place. The pycnocline layer is represented by two boxes ( $t$ and $t s$ ) where the latter is located south of $30^{\circ} \mathrm{S}$.

Density variations in the boxes are solely due to the salinity variations, as the temperature in each box is assumed to be constant. The downwelling in the North Atlantic (and hence the AMOC strength) is represented by the term $q_{N}$, while $q_{S}$ is the difference between the wind-driven Ekman flow $\left(q_{E k}\right)$ and the eddy-induced flow $\left(q_{e}\right)$, the latter associated with baroclinic instabilities of the Antarctic Circumpolar Current. The upwelling volume transport from the deep layer to the pycnocline layer is represented by $q_{U}$. Two coefficients, $r_{N}$ and $r_{S}$, capture the effect of the wind-driven subtropical gyres on the salinity transport. Finally, the Atlantic Ocean circulation model is forced by external freshwater fluxes, split into a symmetric $\left(E_{s}\right)$ and an asymmetric $\left(E_{a}\right)$ component (Fig. 3.1$)$; the noise will only be incorporated through $E_{a}$.

The equations determining the evolution of the AMOC in this model are the salinity budgets of the different boxes, together with the variation of the volume 


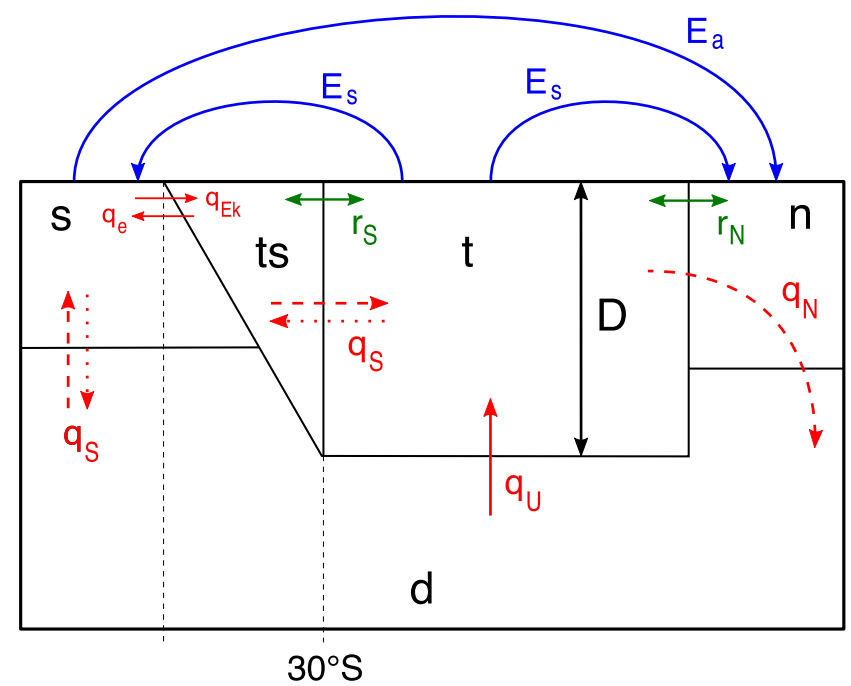

Figure 3.1: Sketch of the box model, adapted from [Cimatoribus et al., 2014]. The red, blue and green arrows represent, respectively, the volume fluxes between the boxes, the freshwater exchange between the basin and the atmosphere, and the effect of the subtropical gyres on the transport. The dashed (dotted) arrows indicate fluxes present only in the AMOC 'on' ('off') state: in the collapsed state, the downwelling $\left(q_{N}\right)$ stops and the transport $q_{S}$ is reversed.

of the pycnocline, and the salt and volume conservation equations:

$$
\begin{aligned}
\frac{\mathrm{d}\left(V_{t} S_{t}\right)}{\mathrm{d} t} & =q_{S}\left(\theta\left(q_{S}\right) S_{t s}+\theta\left(-q_{S}\right) S_{t}\right)+q_{U} S_{d}-\theta\left(q_{N}\right) q_{N} S_{t}+r_{S}\left(S_{t s}-S_{t}\right) \\
& +r_{N}\left(S_{n}-S_{t}\right)+2 E_{s} S_{0}, \\
\frac{\mathrm{d}\left(V_{t s} S_{t s}\right)}{\mathrm{d} t} & =q_{E k} S_{s}-q_{e} S_{t s}-q_{S}\left(\theta\left(q_{S}\right) S_{t s}+\theta\left(-q_{S}\right) S_{t}\right)+r_{S}\left(S_{t}-S_{t s}\right), \\
\frac{\mathrm{d}\left(V_{n} S_{n}\right)}{\mathrm{d} t} & =\theta\left(q_{N}\right) q_{N}\left(S_{t}-S_{n}\right)+r_{N}\left(S_{t}-S_{n}\right)-\left(E_{s}+E_{a}\right) S_{0}, \\
\frac{\mathrm{d}\left(V_{s} S_{s}\right)}{\mathrm{d} t} & =q_{S}\left(\theta\left(q_{S}\right) S_{d}+\theta\left(-q_{S}\right) S_{s}\right)+q_{e} S_{t s}-q_{E k} S_{s}-\left(E_{s}-E_{a}\right) S_{0}, \\
\left(A+\frac{L_{x A} L_{y}}{2}\right) \frac{\mathrm{d} D}{\mathrm{~d} t} & =q_{U}+q_{E k}-q_{e}-\theta\left(q_{N}\right) q_{N}, \\
S_{0} V_{0} & =V_{n} S_{n}+V_{d} S_{d}+V_{t} S_{t}+V_{t s} S_{t s}+V_{s} S_{s},
\end{aligned}
$$

where the function $\theta(x)$ is the Heaviside step function, which is 1 for a positive argument and 0 otherwise. Through this function, we can represent the different circulation given by the different sign of $q_{S}$ and by the possibility that the down- 
welling $\left(q_{N}\right)$ is zero. The last equation expresses the conservation of salinity in the basin. The transports depend, in turn, on the variables, via the following relations:

$$
\begin{aligned}
q_{E k} & =\frac{\tau L_{x S}}{\rho_{0}\left|f_{S}\right|}, \\
q_{e} & =A_{G M} \frac{L_{x A}}{L_{y}} D, \\
q_{U} & =\frac{\kappa A}{D}, \\
q_{N} & =\eta \frac{\rho_{n}-\rho_{t s}}{\rho_{0}} D^{2}, \\
q_{S} & =q_{E k}-q_{e},
\end{aligned}
$$

where the density of the generic box $i$ is defined as

$$
\rho_{i}=\rho_{0}\left(1-\alpha\left(T_{i}-T_{0}\right)+\beta\left(S_{i}-S_{0}\right)\right)
$$

The equation for the downwelling, introduced by Cimatoribus et al. [2014], is based on the northward geostrophic transport above the thermocline in the high latitudes. Thus, $q_{N}$ is determined by the density contrasts between the northern part and southern part of the Atlantic Ocean. The relation was found in several ocean GCM studies [Rahmstorf, 1996, Weijer et al., 2012, den Toom et al., 2014], even with near eddy-resolving resolution. For instance, Fig. 8 in [Rahmstorf, 1996] shows the linear dependence of the NADW (North Atlantic Deep Water) flow on the density difference between two latitudinal strips $\left(50-55^{\circ} \mathrm{N}\right.$ and $\left.35-40^{\circ} \mathrm{S}\right)$ at mid-depth $(750 \mathrm{~m})$. Moreover, this linear dependence was successfully tested in [Levermann and Fürst, 2010].

Also the volume of the pycnocline, as well as the one of the deep box, depend on the state of the system, in particular on the variable $D$ :

$$
\begin{aligned}
V_{t} & =A D, \\
V_{t s} & =\frac{L_{x A} L_{y}}{2} D, \\
V_{d} & =V_{0}-V_{n}-V_{s}-V_{t}-V_{t s} .
\end{aligned}
$$

The reference parameter values, together with their descriptions, are shown in table 3.1.

Two different circulation regimes can be achieved, corresponding to two stable steady states of eqs. (3.1.1): for future reference, they are referred to as AMOC 'on' and 'off' state, respectively (see Fig. 3.1). A detailed study of the equilibria of the system is given in chapter 5 . 


\begin{tabular}{|c|c|c|}
\hline \multicolumn{3}{|r|}{ Parameters used in the model } \\
\hline$V_{0}$ & $3 \times 10^{17} \mathrm{~m}^{3}$ & total volume of the basin \\
\hline$V_{n}$ & $3 \times 10^{15} \mathrm{~m}^{3}$ & volume of the northern box \\
\hline$V_{s}$ & $9 \times 10^{15} \mathrm{~m}^{3}$ & volume of the southern box \\
\hline$A$ & $1 \times 10^{14} \mathrm{~m}^{2}$ & horizontal area of the Atlantic pycnocline \\
\hline$L_{x A}$ & $1 \times 10^{7} \mathrm{~m}$ & zonal extent of the Atlantic Ocean at its southern end \\
\hline$L_{y}$ & $1 \times 10^{6} \mathrm{~m}$ & meridional extent of the frontal region of the Southern Ocean \\
\hline$L_{x S}$ & $3 \times 10^{7} \mathrm{~m}$ & zonal extent of the Southern Ocean \\
\hline$\tau$ & $0.1 \mathrm{~N} \mathrm{~m}^{-2}$ & average zonal wind stress amplitude \\
\hline$A_{G M}$ & $1700 \mathrm{~m}^{2} \mathrm{~s}^{-1}$ & eddy diffusivity \\
\hline$f_{S}$ & $-10^{-4} \mathrm{~m}^{3}$ & Coriolis parameter \\
\hline$\rho_{0}$ & $1027.5 \mathrm{~kg} \mathrm{~m}^{-3}$ & reference density \\
\hline$\kappa$ & $10^{-5} \mathrm{~m}^{2} \mathrm{~s}^{-1}$ & vertical diffusivity \\
\hline$S_{0}$ & $35 \mathrm{psu}$ & reference salinity \\
\hline$T_{0}$ & $5 \mathrm{~K}$ & reference temperature \\
\hline$T_{n}$ & $5 \mathrm{~K}$ & temperature of the northern box \\
\hline$T_{t s}$ & $10 \mathrm{~K}$ & temperature of the box $t s$ \\
\hline$\eta$ & $3 \times 10^{4} \mathrm{~m} \mathrm{~s}^{-1}$ & hydraulic constant \\
\hline$\alpha$ & $2 \times 10^{-4} \mathrm{~K}^{-1}$ & thermal expansion coefficient \\
\hline$\beta$ & $8 \times 10^{-4} \mathrm{psu}^{-1}$ & haline contraction coefficient \\
\hline$r_{S}$ & $1 \times 10^{7} \mathrm{~m}^{3} \mathrm{~s}^{-1}$ & transport by the southern subtropical gyre \\
\hline$r_{N}$ & $5 \times 10^{6} \mathrm{~m}^{3} \mathrm{~s}^{-1}$ & transport by the northern subtropical gyre \\
\hline$E_{s}$ & $0.17 \times 10^{6} \mathrm{~m}^{3} \mathrm{~s}^{-1}$ & symmetric freshwater flux \\
\hline
\end{tabular}

Table 3.1: Reference parameters used in eqs. (3.1.1) - (3.1.4).

\subsubsection{An indicator for the AMOC stability}

Following initial ideas by Rahmstorf [1996] and de Vries and Weber [2005], a well-known indicator for the present-day AMOC stability is based on the AMOCinduced freshwater divergence in the Atlantic, referred to as $\Sigma$ in [Dijkstra, 2007], later reintroduced as $\Delta M_{o v}$ by [Liu et al., 2014, 2017]:

$$
\Sigma\left(\theta_{n}, \theta_{s}\right)=M_{o v}\left(\theta_{s}\right)-M_{o v}\left(\theta_{n}\right)
$$


where $\theta_{n}$ and $\theta_{s}$ are the northern and southern latitudes of the Atlantic domain. $M_{o v}$ is defined as follows:

$$
M_{o v}(\theta)=-\frac{\eta}{S_{0}} \int\langle v\rangle\left(\langle S\rangle-S_{0}\right) \mathrm{d} z
$$

with $\eta$ given by $\eta=\int r_{0} \cos \theta \mathrm{d} \phi$, where $r_{0}, \theta$ and $\phi$ represent, respectively, radius of the Earth, latitude and longitude, and $\langle\cdot\rangle=\frac{1}{\eta} \int \cdot r_{0} \cos \theta \mathrm{d} \phi$.

A negative value of $\Sigma$ indicates that the overturning circulation transports freshwater out of the Atlantic basin. If the AMOC weakens due to a perturbation, less freshwater is transported out of the basin; therefore, the Atlantic Ocean becomes fresher, which results in a further weakening of the AMOC. In other terms, the AMOC is sensitive to finite-amplitude perturbations, that can lead to its collapse. When $\Sigma$ is positive, salt is transported out of the Atlantic Ocean and a negative feedback is established as a result of a perturbation. Therefore, $\Sigma$ can serve as an indicator for the AMOC stability, at least when the atmospheric fluxes are considered constant: a negative (positive) value of $\Sigma$ indicates that the AMOC is in a multiple (single) equilibria regime. The component at the southern boundary of the Atlantic (at $35^{\circ} \mathrm{S}$ ) happens to be much larger than the northern component (at $65^{\circ} \mathrm{N}$ ) [de Vries and Weber, 2005, Huisman et al., 2010], hence $M_{o v}\left(\theta_{s}\right) \equiv M_{o v}$ is often used instead of $\Sigma$.

Thanks to the splitting of the pycnocline layer in our model, it is possible to isolate the overturning contribution to the freshwater transport at the southern boundary of the Atlantic ocean, corresponding to the southern border of the $t s$ box. The indicator is then given by

$$
M_{o v}=-\frac{q_{S}}{S_{0}}\left(S_{t s}-S_{d}\right)
$$

\subsubsection{Atmospheric freshwater noise}

The amplitude of the atmospheric noise forcing in the model is estimated from observational data relative to the freshwater forcing in the Atlantic Ocean. The values the P-E (precipitation minus evaporation) were year-averaged and integrated over two basins, corresponding to the northern and southern boxes in the model. The time series thus obtained were summed and subtracted from each other, resulting respectively in the symmetric and antisymmetric component of the freshwater forcing, namely $E_{s}$ and $E_{a}$ in the model (see Fig. 3.2). The value of the noise (referred to as $f_{\sigma}$ ) is then computed as the ratio between the standard deviation and the mean value of $E_{a}$. Such value is representative of the interannual time scale variability of the forcing. If more high-frequency noise is used, ocean mixed-layer processes (not represented in the box model) will integrate this noise to give interannual variability in the surface salinity affecting the North-South density gradient. Hence, in our box model these interannual variations are an adequate description 
of unresolved processes. As a first order approximation of the process, we assume the noise to be white and normally distributed. In [Ganopolski and Rahmstorf, 2002], a similar choice was made, given that the decorrelation time of the stochastic freshwater forcing, computed by their model, is one year. We use $f_{\sigma}$ as the lower bound of the noise amplitude, as, if smaller time scales are considered, the associated variability increases.

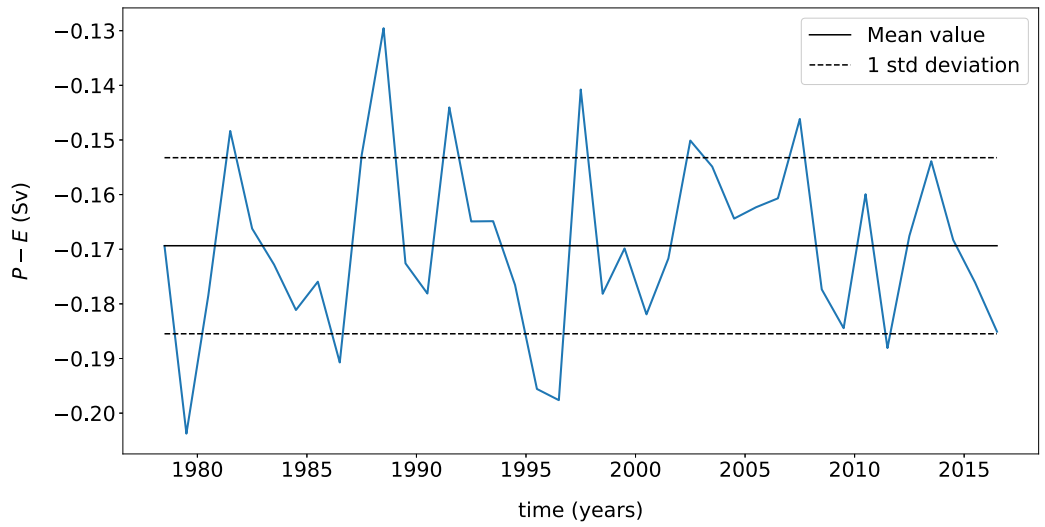

Figure 3.2: From the observed yearly averaged P-E in the Atlantic Ocean, from ERA-Interim Archive at ECMWF [Dee et al., 2011], its antisymmetric part is shown, respect to the North $\left(50^{\circ} \mathrm{N}-70^{\circ} \mathrm{N}\right)$ and South (South of $\left.40^{\circ} \mathrm{S}\right)$ Atlantic. The black horizontal lines indicate the mean value (solid line) and one standard deviation confidence interval (dashed lines). The value of $f_{\sigma}$, that is the ratio between the standard deviation and the mean value, is about 0.1 . 


\subsection{Transition probabilities of noise-induced transi- tions}

Estimating transition probabilities for transitions between different states in a generic stochastic system is a challenging task.

Consider a system of SDEs (1.2.1) and assume that the corresponding deterministic system has two stable steady states, $\mathbf{X}_{A}$ and $\mathbf{X}_{B}$ and a saddle point $\mathbf{X}_{C}$, for a certain choice of the parameters. The system is subject to a noise term, with noise matrix $G=\sigma I$. Our goal is to compute transition probabilities for transitions between $\mathbf{X}_{A}$ and $\mathbf{X}_{B}$, defined as the probability $P\left(\tau_{B}<T \mid \mathbf{X}_{0}=\mathbf{X}_{A}\right)$ that a trajectory starting in $\mathbf{X}_{A}$ reaches a neighbourhood $B$ around $\mathbf{X}_{B}$ before time $T$.

Analytical results are only available for very specific low-dimensional dynamical systems, namely when the drift term in eq. (1.2.1) can be written as the gradient of a certain potential $F=-\nabla V$. Such results are based on the Eyring-Kramers formula [Eyring, 1935, Kramers, 1940], which gives an estimation the mean first passage time, that is the average time it takes to reach $\mathbf{X}_{B}$ from $\mathbf{X}_{A}$ at a noise level $\sigma:$

$$
\mathbb{E}\left[\tau_{\mathbf{X}_{A} \rightarrow \mathbf{X}_{B}}^{\sigma}\right] \underset{\sigma \rightarrow 0}{\sim} \frac{2 \pi}{-\lambda_{C}} \sqrt{\frac{\left|\operatorname{det}\left(\nabla^{2} V\left(\mathbf{X}_{C}\right)\right)\right|}{\operatorname{det}\left(\nabla^{2} V\left(\mathbf{X}_{A}\right)\right)}} e^{\frac{V\left(\mathbf{X}_{C}\right)-V\left(\mathbf{X}_{A}\right)}{\sigma^{2} / 2}},
$$

where $\lambda_{C}$ is the single negative eigenvalue of the Hessian matrix $\nabla^{2} V\left(\mathbf{X}_{C}\right)$. This is reminiscent of Arrhenius' law [Arrhenius, 1889], which states that the rate constant $\lambda$ of a chemical reaction scales like $\lambda(T) \sim e^{-E_{a} /\left(k_{B} T\right)}$, where $E_{a}$ is the activation energy, $k_{B}$ the Boltzmann constant and $T$ the temperature. Here, the noise parameter $\sigma$ plays the role of the temperature. For a Poisson process (which assumes transitions to be instantaneous and independently scattered), the probability of having a transition in an infinitesimal interval of time $d t$ is then given by $d t / \mathbb{E}\left[\tau_{\mathbf{X}_{A} \rightarrow \mathbf{X}_{B}}^{\sigma}\right]$. By taking $d t=T / N$ for $N \rightarrow \infty$, the transition probability in a fixed time T is then:

$$
\begin{aligned}
P\left(\tau_{B}<T \mid \mathbf{X}_{0}=\mathbf{X}_{A}\right) & =1-P\left(\mathbf{X}_{t} \notin B, \forall t \in[0, T] \mid \mathbf{X}_{0}=\mathbf{X}_{A}\right) \\
& =1-\lim _{N \rightarrow \infty}\left(1-\frac{T}{\mathbb{E}\left[\tau_{\mathbf{X}_{A} \rightarrow \mathbf{X}_{B}}^{\sigma}\right] N}\right)^{N} \\
& =1-e^{-T / \mathbb{E}\left[\tau_{\mathbf{X}_{A} \rightarrow \mathbf{X}_{B}}^{\sigma}\right]}
\end{aligned}
$$

By substituting eq. (3.2.1) into the previous equation, we can finally express the transition probability as a function of the noise $\sigma$ and the potential $V$. The Eyring-Kramers formula has been generalised to non-gradient systems [Bouchet and Reygner, 2016], where the quasi-potential plays the role of the potential. The procedure described here has some limitations: first of all, eq. (3.2.1) is valid only when white additive noise is imposed to the system, and it is exact only in the limit of small noise $(\sigma \rightarrow 0)$; second, the transitions occurring in a generic system cannot always be well represented by Poisson processes. Moreover, the method suggested 
by Bouchet and Reygner [2016] for non-gradient systems (the large majority of the physical systems) requires considerable computational efforts, and it is often not feasible, especially for high-dimensional systems.

\subsubsection{Rare event algorithms}

From the numerical point of view, transition probabilities can be computed, in principle, using a Monte Carlo approach, namely by performing simulations of large ensembles of trajectories and counting the number of trajectories which actually undergo a transition. However, if the occurrence of a transition is a rare event, such computations are not feasible. Indeed, for instance, to sample an event of probability $p \sim 10^{-8}$, one would need to compute at least $N>N_{\min }=10^{8}$ trajectories $\left(N_{\min } \sim 1 / p\right)$, which is computationally impossible to achieve for largedimensional dynamical systems, where time integrations are expensive.

In order to sample tails of distributions more effectively, various methods have been developed, generally referred to as rare-event algorithms. One of the promising methods to compute transition probabilities is the Trajectory-Adaptive Multilevel Sampling (TAMS) method [Lestang et al., 2018]. Its underlying idea is to perform a selection/mutation process that discards trajectories going away from a certain target set and splits/branches from those that get closer to this set. A very similar algorithm, Adaptive Multilevel Splitting (AMS) based on the same mechanism, has been used in the study of transitions in Jupiter's turbulent dynamics [Bouchet et al., 2019] and in molecular dynamics to compute the expected dissociation time between a protein and its ligand [Teo et al., 2016]. In these studies, AMS proved to be a powerful tool that improved computational capabilities by several orders of magnitude. Indeed, the required minimum number of computed trajectories scales like $N_{\min } \sim 1 / \log p$ [Cérou et al., 2016], which is exponentially better than that for the Monte Carlo estimation. Furthermore, it has to be noticed that these algorithms do not require specific assumptions on the structure (state dependent or not) or color of the noise.

\subsubsection{TAMS algorithm}

The Trajectory-Adaptive Multilevel Splitting algorithm [Lestang et al., 2018] is based on the idea of simulating a large ensemble of trajectories, and discarding the ones that do not reach the final state and splitting (or branching) trajectories that are closer to it. As a consequence, the probability that a trajectory reaches the final state keeps increasing, which is why this method is more efficient than brute-force techniques.

At each time step, the trajectories are ranked according to a so-called score function, which gives a measure of how close the trajectory is to the final state. The ones with the lowest rank are discarded, while new ones are generated, by randomly branching other trajectories. A certain weight, which is related to the 
number of discarded trajectories at each time iteration, is a measure of the probability of a trajectory to reach the next step. An unbiased estimator of the transition probability $\hat{p}$ can be obtained multiplying these weights. A step-by-step description of the method is given in the next page.
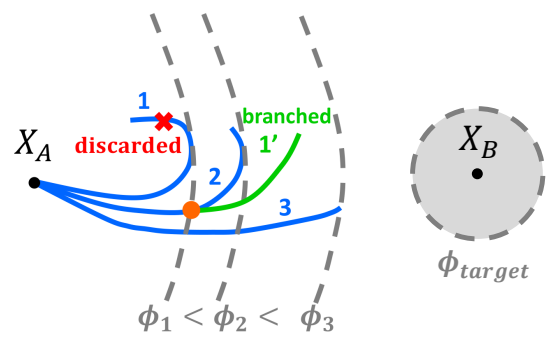

Figure 3.3: Illustration of the TAMS algorithm. First, simulate $N$ trajectories starting in $\mathbf{X}_{A}$. ( $N=3$ blue trajectories in the figure). The trajectories are ranked according to their score $\phi_{i}$, which is the maximum value of the user-supplied score function $\phi$ along the trajectory. Then, at each iteration, the trajectory with lowest score ( 1 in the figure, with score $\phi_{1}$ ) is discarded. It is replaced by picking a trajectory uniformly at random from the other ones ( 2 in the figure), then computing the earliest position at which it reaches a higher score than the discarded trajectory (orange dot) and finally using this position as the branching point for the new trajectory $\left(1^{\prime}\right)$. Repeat until all the trajectories reach $B$ or the number of iterations $k$ reaches a predefined limit $k_{\max }$.

Note also that in the version of TAMS we use, the trajectories having the smallest score are discarded. However, one can also choose to discard those which have the $K>1$ smallest scores, which leads to an unbiased estimator as well. A good score function is crucial for the convergence and the performance of the algorithm [Rolland and Simonnet, 2015]. In the next chapter several choices are discussed in detail. 


\section{Trajectory-Adaptive Multilevel sampling (TAMS) algorithm}

Input: $\quad N \quad$ number of trajectories in the ensemble

$T \quad$ time at which the probability is eventually calculated

$k_{\max } \quad$ maximum number of iterations of the algorithm

$\phi \quad$ score function

$\phi_{\text {target }}$ target score: the condition that defines the occurrence of a transition

Output: $\quad \hat{p} \quad$ transition probability estimation

\section{Initialization:}

1: for $\mathrm{i}=1, \ldots, \mathrm{N}$ do

2: $\quad$ Simulate the trajectory $\left(\mathbf{X}^{(i)}\right)=\left(\mathbf{X}_{0}^{(i)}, \ldots, \mathbf{X}_{T}^{(i)}\right)$ of the different ensemble members $i$ (e.g. using the Euler-Maruyama scheme).

3: $\quad$ Compute the score $\phi_{i} \equiv \max _{0 \leq t \leq T} \phi\left(\mathbf{X}_{t}^{(i)}\right)$ of trajectory $i$, which is the maximum value of the score function $\phi$ along the trajectory $\mathrm{i}$.

4: end for

5: Set the iteration number $k=1$

\section{Main loop:}

6: while $\min _{1 \leq i \leq N} \phi_{i}<\phi_{\text {target }}$ and $k<k_{\max }$ do

7: $\quad$ Discard the ensemble members for which the trajectories realised the minimum score i.e discard the indices in $S_{k} \equiv\left\{j \mid \phi_{j}=\min _{1 \leq i \leq N} \phi_{i}\right\}$.

8: $\quad$ Set $l_{k} \equiv \operatorname{Card}\left(S_{k}\right)$ the number of discarded trajectories $\left(S_{k}=\{1\}\right.$ and $l_{k}=1$ in Fig. 3.3. Note that $S_{k}$ can have multiple elements because of the time discretization).

9: $\quad$ for $j$ in $S_{k}$ do

10: Choose uniformly at random a trajectory index $r$ in $\{1, \ldots, N\} \backslash S_{k}$. This is the trajectory from which the new trajectory will originate from ( $r=2$ in Fig. 3.3).

11: $\quad$ Copy the trajectory $\left(\mathbf{X}^{(r)}\right)$ into the new trajectory $\left(\widetilde{\mathbf{X}}^{(j)}\right)$ up to the first time the value of the score function is greater than $\phi_{j}$. In other words, set $\left(\widetilde{\mathbf{X}}_{0}^{(j)}, \ldots, \widetilde{\mathbf{X}}_{t_{\text {branch }}}^{(j)}\right) \equiv$ $\left(\mathbf{X}_{0}^{(r)}, \ldots \mathbf{X}_{t_{\text {branch }}}^{(r)}\right)$ where $t_{\text {branch }} \equiv \min \left(\left\{0 \leq t \leq T \mid \phi\left(\mathbf{X}_{t}^{(r)}\right) \geq \phi_{j}\right\}\right)$.

12: $\quad$ Generate the rest of the new trajectory starting from $\widetilde{\mathbf{X}}_{t_{\text {branch }}}^{(j)} \equiv \mathbf{X}_{t_{\text {branch }}}^{(r)}$ (orange dot in Fig. 3.3) until time $T$ (green trajectory in Fig. 3.3).

13: $\quad$ Replace the discarded trajectory $\left(\mathbf{X}^{(j)}\right) \leftarrow\left(\widetilde{\mathbf{X}}_{0}^{(j)}, \ldots, \widetilde{\mathbf{X}}_{t_{\text {branch }}}^{(j)}, \ldots, \widetilde{\mathbf{X}}_{T}^{(j)}\right)$.

14: $\quad$ Update the corresponding score $\phi_{j} \leftarrow \max _{t_{\text {branch }} \leq t \leq T} \phi\left(\mathbf{X}_{t}^{(j)}\right)$ which is greater than or equal to the previous score (by construction of $t_{\text {branch }}$ ).

15: $\quad$ end for

16: $\quad k \leftarrow k+1$

17: end while

18: Set $N_{B}$ the number of trajectories having reached the target set $B$, i.e that have a score greater than $\phi_{\text {target }}$.

19: return

$$
\hat{p}=\frac{N_{B}}{N} \prod_{i=0}^{k}\left(1-\frac{l_{i}}{N}\right)
$$





\section{Chapter 4}

\section{The importance of score functions in the TAMS algorithm}

As we have mentioned, the Trajectory-Adaptive Multilevel Sampling (TAMS) algorithm is a promising method to determine probabilities of noise induced transition in multi-stable dynamical systems. In this chapter, we focus on two improvements of the current algorithm related to (i) the choice of the target set and (ii) the formulation of the score function. In particular, we use confidence ellipsoids, determined from linearised dynamics, in the choice of the target sets. Furthermore, we define a score function based on empirical transition paths computed at relatively high noise levels. The suggested improved TAMS method is applied to the AMOC box model introduced in the previous chapter.

This chapter is based on the following manuscript:

Wang, P., Castellana, D. and Dijkstra, H. A. (2020). Improvements to the use of the Trajectory-Adaptive Multilevel Sampling algorithm for the study of rare events. Manuscript under review. 


\subsection{Introduction}

The selection and mutation process of discarding and branching trajectories in TAMS is carried out according to a score function, which allows to rank trajectories at each time step. Rolland and Simonnet [2015] have shown that the choice of the score function plays an important role for the performance of the algorithm, even for systems with only two degrees of freedom. When using non-optimal score functions, especially near phase transitions, the variance of the estimated probability can peak and the convergence of the algorithm can be slow.

The aim of this chapter is to propose improvements to the use of the TAMS algorithm to be able to compute transitions in multi-stable systems more efficiently. The first type of improvement is the choice of the target set, which is often determined from rather arbitrary thresholds. This choice also raises more broadly the question of a precise definition of what we consider a noise-induced transition between two (stable) states. The second type of improvement regards the choice of the score function: we propose a more systematic method of defining a score function, based on empirical transition paths. The modified TAMS method is first applied to an idealised gradient systems, and then to the AMOC box model presented in the previous chapter.

In section 4.2, we describe the methods developed to improve the TAMS algorithm. In section 4.3 we show how to incorporate these techniques into the definition of the score function and present the results for idealised dynamical systems and the low-dimensional AMOC model. A discussion follows in section 4.4, indicating the strengths and the limitations of our approach.

\subsection{Methods}

\subsubsection{Transition probabilities using TAMS}

We consider finite-dimensional dynamical systems described by stochastic differential equations of the following form:

$$
\mathrm{d} \mathbf{X}_{t}=F\left(\mathbf{X}_{t}\right) \mathrm{d} t+G \mathrm{~d}_{t},
$$

where $\mathbf{X}_{t} \in \mathbb{R}^{n}$ and $F: \mathbb{R}^{n} \rightarrow \mathbb{R}^{n}$ is the drift field. The noise term $\mathbf{W}_{t} \in \mathbb{R}^{m}$ consists of $m$ independent Wiener processes with the matrix $G: \mathbb{R}^{n} \times \mathbb{R}^{m}$ being the noise matrix. The equation above is a particular case of the more general expression (1.2.1): in this chapter we only consider systems subject to additive noise. However, the methods presented can be easily adapted to include the multiplicative case.

Assume that the system has two stable steady states, $\mathbf{X}_{A}$ and $\mathbf{X}_{B}$. Without fluctuations, a trajectory starting in $\mathbf{X}_{A}$ stays in $\mathbf{X}_{A}$. However, because of the presence of noise, modelled by the noise term in the SDE, it can move away from $\mathbf{X}_{A}$ and, in some cases, make the transition to the other stable state $\mathbf{X}_{B}$. 
A prominent example of the system (4.2.1) is a model of a free particle moving in a double-well potential system, with $n=m=2$. The drift term in the timeevolution equation for the variables $x$ and $y$ is in this case

$$
F(x, y) \equiv-\nabla V(x, y)=\left(x-x^{3},-y\right)
$$

where $V(x, y)$ represents the potential (Fig. 4.1(a)). In the deterministic case (i.e. $G=0)$, the stable steady states of the system are $\mathbf{X}_{A}=(-1,0)$ and $\mathbf{X}_{B}=(+1,0)$, while the unstable steady state is $\mathbf{X}_{C}=(0,0)$.

We would like to compute the transition probability, in a certain time $T$, of the occurrence of a noise-induced transition, using the TAMS algorithm. In order to properly characterise transitions, we need to define a target set $(B)$, that is a small (at first, arbitrary) neighbourhood of $\mathbf{X}_{B}$. In Fig. 4.1(b) an example of noiseinduced transition is represented.
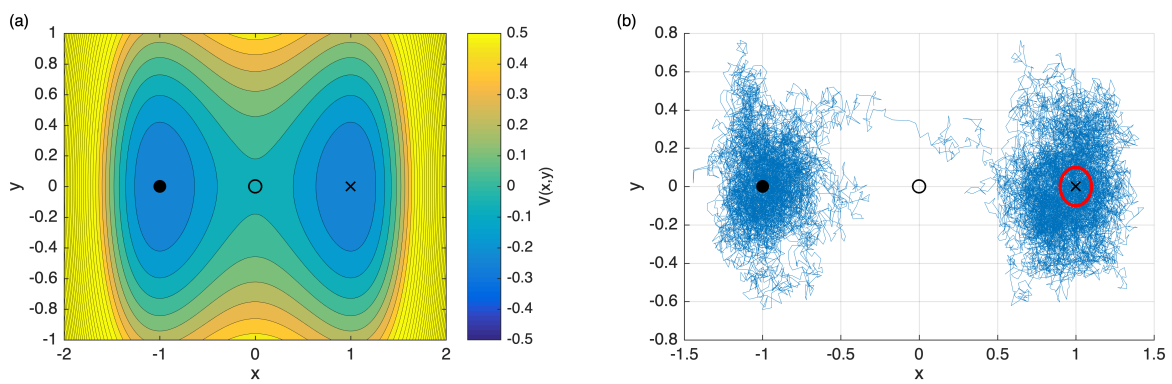

Figure 4.1: (a) Iso-potential contours corresponding to the double-well potential (4.2.2). The two stable steady states $\mathbf{X}_{A}=(-1,0)$ and $\mathbf{X}_{B}=(+1,0)$ are marked with a filled circle and a cross, respectively. The saddle $\mathbf{X}_{C}=(0,0)$ is indicated by a circle. (b) Example of a noise-induced transition from $\mathbf{X}_{A}$ to $\mathbf{X}_{B}$ for a particle moving in the double-well potential in (a). The noise matrix in the general SDE (4.2.1) is chosen as $G=\sigma I$, with $\sigma=0.32$. The red circle denotes an arbitrarily defined target set $B$.

\subsubsection{Different score functions}

As already mentioned in the previous chapter, the TAMS algorithm needs a score function to be defined, which allows to rank trajectories, and to select the ones to discard. The optimal score function $\phi_{c o m}(\mathbf{X}, t)$, i.e. the score function that minimises the variance of the probability estimator, is called the static committor. Its generic expression is given by the following conditional probability [Lestang et al., 2018]:

$$
\phi_{\text {com }}(\mathbf{X}, t)=P\left(\tau_{B}<T \mid \mathbf{X}, t\right)
$$

where $T$ is the fixed duration of the trajectories and $\tau_{B}$ is the stopping time associated with reaching the target set $B$. In other words, $\phi_{\text {com }}(\mathbf{X}, t)$ is the probability that a trajectory starting in $\mathbf{X}$ at time $t$ reaches $B$ before time $T$. This expression is 
quite natural because, ideally, the score of $(\mathrm{Y}, s)$ should be higher than the score of (X, $t)$, i.e., $\phi(\mathbf{X}, t) \leq \phi(\mathbf{Y}, s)$, if and only if $P\left(\tau_{B}<T \mid \mathbf{X}, t\right) \leq P\left(\tau_{B}<T \mid \mathbf{Y}, s\right)$. This condition is clearly satisfied by $\phi_{\text {com }}$ (and in fact any increasing function of $\phi_{\text {com }}$ ). However, the expression given in eq. (4.2.3) is generally unusable because it is the very quantity that we want to compute. For instance, $\phi_{\text {com }}\left(\mathbf{X}_{A}, 0\right)$ is precisely the transition probability that TAMS estimates.

As a conditional probability of the form $P(\mathbf{Y}, s \mid \mathbf{X}, t)$, the committor $\phi_{\text {com }}(\mathbf{X}, t)$ satisfies the backward Fokker Planck equation [Lestang et al., 2018]:

$$
\frac{\partial \phi}{\partial t}+F \cdot \nabla \phi+\frac{1}{2} \sum_{i, j} G_{i j} \frac{\partial^{2} \phi}{\partial X_{i} \partial X_{j}}=0 \quad\left\{\begin{array}{l}
\forall \mathbf{X} \in \partial B, \forall t \in[0, T], \phi(\mathbf{X}, t)=1 \\
\forall \mathbf{X} \in \mathbb{R}^{n} \backslash B, \phi(\mathbf{X}, T)=0
\end{array}\right.
$$

However, solving the backward Fokker Plank equation in systems with many degrees of freedom is computationally infeasible. Moreover, even if the committor is available on a discretised grid, using interpolation to evaluate it during a TAMS loop can also have a prohibitive computational cost.

Therefore Bouchet et al. [2019] proposed a score function based on the distances of the state $\mathbf{X}$ from the starting state $\mathbf{X}_{A}$ and the destination equilibrium $\mathbf{X}_{B}$, respectively: it is defined as

$$
\phi_{\text {dist }}(\mathbf{X}) \equiv\left\{\begin{array}{ll}
d_{A} / 2 d_{B} & \text { if } d_{A}<d_{B} \\
1-d_{B} / 2 d_{A} & \text { otherwise }
\end{array} \quad \text { with } d_{A} \equiv\left\|\mathbf{X}-\mathbf{X}_{A}\right\|, d_{B} \equiv\left\|\mathbf{X}-\mathbf{X}_{B}\right\|\right.
$$

Alternatively, a Gaussian-shaped score function $\phi_{\text {gauss }}$ was proposed in [Baars, 2019]. It is defined as:

$$
\phi_{\text {gauss }}(\mathbf{X}) \equiv \eta-\eta e^{-\beta\left\|\mathbf{X}-\mathbf{X}_{A}\right\|^{2} /\left\|\mathbf{X}_{C}-\mathbf{X}_{A}\right\|^{2}}+(1-\eta) e^{-\beta\left\|\mathbf{X}-\mathbf{X}_{B}\right\|^{2} /\left\|\mathbf{X}_{C}-\mathbf{X}_{B}\right\|^{2}},
$$

with $\eta \equiv\left\|\mathbf{X}_{C}-\mathbf{X}_{A}\right\| /\left\|\mathbf{X}_{A}-\mathbf{X}_{B}\right\|$. Here $\beta \in \mathbb{R}$ is a parameter controlling the decay and $\mathbf{X}_{C}$ is the saddle state of the system. Due to the general expressions of $\phi_{\text {dist }}$ and $\phi_{\text {gauss }}$, these score functions can be used in systems of any dimension.

\subsubsection{Definition of the target set}

As already mentioned, once the score function has been chosen, a threshold needs to be defined for the TAMS algorithm to converge, so that the occurrence of a transition can be detected. In other words, we do not expect each trajectory that undergoes a transition to reach exactly the destination equilibrium $\mathbf{X}_{B}$, but rather a neighbourhood of it $(B)$. The target set $B$ can then be defined according to a level set $\phi^{\text {target }}$ of the score function $\phi$ :

$$
B=\left\{\mathbf{X} \in \mathbb{R}^{n} \mid \phi(\mathbf{X})>\phi^{\text {target }}\right\} .
$$

However, different score functions $\phi$ and different level sets $\phi^{\text {target }}$ correspond to different target sets $B$, which can differ in volume and in shape. Moreover, 
often the level set $\phi^{\text {target }}$ is defined somewhat arbitrarily. For example, by using $\phi_{\text {gauss }}$ with target score $\phi^{\text {target }}=0.85,0.9$ or 0.95 , we found that the average of the transition probability estimator for a two-dimensional double-well potential system can vary up to $30 \%$. This may not be a concern if one only cares about the order of magnitude of the transition probability but it can be problematic if quantitative comparisons are needed. Moreover, a poor choice of the target set can lead to inaccuracies when a trajectory has a score greater than $\phi^{\text {target }}$ without actually making the transition in all the degrees of freedom. Thus, there is a need for defining a canonical choice of target set $B$, which is fundamentally equivalent to defining what is considered a noise-induced transition.

For this purpose, we use the concept of confidence ellipsoid. This is an ellipsoidal neighbourhood around a stable equilibrium state, inside which a trajectory subject to the locally linearised dynamics stays, within a certain confidence level [Cowan, 1998]. For example, as illustrated in Fig. 4.2, when initializing a trajectory at $\mathbf{X}_{A}$ or $\mathbf{X}_{B}$ in the two-dimensional double-well system, it stays inside the correspondent ellipsoid with a certain confidence $1-\alpha=0.95$. Consider a gen-

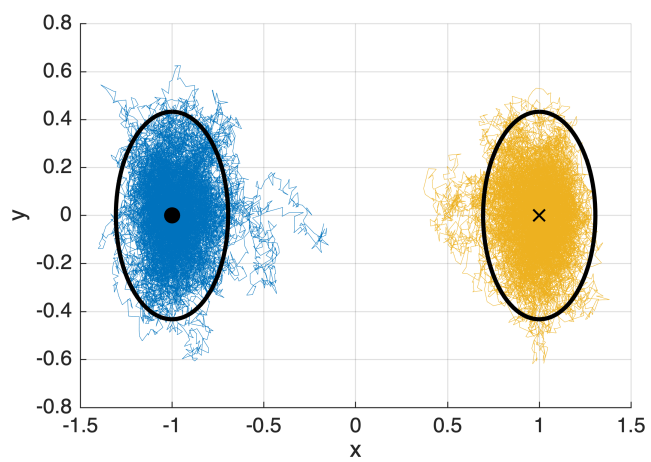

Figure 4.2: Confidence ellipsoids for a two-dimensional double-well potential system, with confidence level $1-\alpha=0.95$ and noise matrix $G=0.25 I$, where $I$ is the identity matrix in $\mathbb{R}^{2}$. Two trajectories of duration $T=200$ are shown: one (blue) is initialised at the initial state and one (orange) at the target state. $93 \%$ of the points composing a trajectory are inside their corresponding ellipsoid. This is lower than the prescribed confidence level because, away from the equilibrium, the first order dynamics from which the confidence ellipsoids are derived does not hold.

eral SDE system given by (4.2.1). Because the drift vanishes at an equilibrium state $F\left(\mathbf{X}_{B}\right)=0$, its first order approximation around the equilibrium state $\mathbf{X}_{B}$ via Taylor expansion is:

$$
F(\mathbf{X})=A\left(\mathbf{X}_{B}\right)\left(\mathbf{X}-\mathbf{X}_{B}\right)+\mathcal{O}\left(\left\|\mathbf{X}-\mathbf{X}_{B}\right\|^{2}\right)
$$

where $A\left(\mathbf{X}_{B}\right)=\nabla F\left(\mathbf{X}_{B}\right) \in \mathbb{R}^{n \times n}$ is the Jacobian matrix of $F$ at $\mathbf{X}_{B}$. Using a transla- 
tion $\widetilde{\mathbf{X}}=\mathbf{X}-\mathbf{X}_{B}$, the first order approximation of the SDE is then:

$$
\mathrm{d} \widetilde{\mathbf{X}_{t}}=A\left(\mathbf{X}_{B}\right) \widetilde{\mathbf{X}_{t}} \mathrm{~d} t+G \mathrm{dW}_{t}
$$

Because the drift term has been linearised, this is the equation for a $n$-dimensional Ornstein-Uhlenbeck process. The stationary probability density function (PDF) $f$ of the approximating process is Gaussian [Cowan, 1998] and given by

$$
f(\mathrm{X})=\frac{1}{(2 \pi)^{\frac{n}{2}}\left|C_{B}\right|^{\frac{1}{2}}} e^{-\frac{1}{2}\left\|\mathrm{X}-\mathbf{X}_{B}\right\|_{C_{B}^{-1}}^{2},}
$$

where $C_{B} \in \mathbb{R}^{n \times n}$ is the covariance matrix of the system calculated in $\mathbf{X}_{B}$ and $\|\cdot\|_{C_{B}^{-1}}$ the norm induced by its inverse $C_{B}^{-1}$, defined by $\|\mathbf{X}\|_{C_{B}^{-1}}^{2} \equiv \mathbf{X}^{\top} C_{B}^{-1} \mathbf{X}$. The covariance matrix $C_{B}$ can be thought heuristically as the matrix containing the correlations $\mathbb{E}\left(x_{i} x_{j}\right)$ (with $\mathbf{X}=\left(x_{1}, \ldots, x_{n}\right)$ ), which generalises the notion of variance in $\mathrm{n}$ dimensions. $C_{B}$ is obtained by solving the Lyapunov equation [see Kuehn, 2012, for the full derivation]:

$$
A\left(\mathbf{X}_{B}\right) C_{B}+C_{B} A\left(\mathbf{X}_{B}\right)^{\top}+G G^{\top}=0 .
$$

We then define the confidence ellipsoid, which has $C_{B}^{-1}$ as shape matrix, as follows

$$
\mathcal{E}=\left\{\mathbf{X} \in \mathbb{R}^{n} \mid\left\|\mathbf{X}-\mathbf{X}_{B}\right\|_{C_{B}^{-1}}^{2} \equiv\left(\mathbf{X}-\mathbf{X}_{B}\right)^{\top} C_{B}^{-1}\left(\mathbf{X}-\mathbf{X}_{B}\right)<Q_{\alpha}\right\}
$$

where $Q_{\alpha}$ is the quantile of confidence level $1-\alpha$ of the $n$-dimensional $\chi^{2}$ distribution [Cowan, 1998]. We usually choose $1-\alpha=0.95$. The directions of symmetry of the ellipsoids are given by the eigenvectors of the covariance matrix $C_{B}$ and the radii are given by the corresponding eigenvalues and the confidence level $1-\alpha$. Intuitively, the greater the eigenvalue, the more a trajectory fluctuates in the given direction.

The $(1-\alpha)$-covariance ellipsoid represents the $n$-dimensional volume where a trajectory is confined with confidence level $1-\alpha$, provided its dynamics is well approximated by the first order expansion at the equilibrium point. An illustration of the 0.95-confidence ellipsoid for the double-well potential is shown in Fig. 4.2. The confidence ellipsoid $\mathcal{E}$ constitutes a way to meaningfully define the target set $B$ with minimal arbitrary parameters. In the next section, we show how to incorporate this choice of target set in the score function $\phi$.

\subsubsection{Estimating the typical transition path using histograms}

The second line of improvement of the score function concerns the estimation of typical transition paths of the dynamical system. In the zero noise limit, the Freidlin-Wentzell theory of large deviations predicts that transition paths cluster around the most probable transition path, called the instanton [Freidlin and 
Wentzell, 1984]. On the other hand, in the finite noise regime, transition paths may deviate from the instanton. Moreover, instantons may be computationally inaccessible for systems with many degrees of freedom. Therefore, it can prove more relevant to estimate empirically the typical transition path that the system follows at a given finite noise level, which is the approach we follow here.

The idea is to first accumulate transition paths at a noise level where transitions are frequent enough (typically $p>10^{-3}$ ) so that any sampling method (direct Monte Carlo or TAMS with naive score functions) can be used. Then, we compute the spatial histogram of the transition paths over a discretised phase space using n-dimensional boxes. This provides the spatial distribution of the transition paths, which is concentrated around a typical transition path, reminiscent of an instanton phenomenology, which was also observed in more complex systems [Bouchet et al., 2019]. From the spatial histogram, we extract a typical transition path. The main steps of the path-finding algorithm are listed below:

(i) the trajectory of the typical transition path starts in the box of the histogram containing the initial state $\mathbf{X}_{A}$;

(ii) the next box in the trajectory corresponds to the neighbour which has the highest nonzero histogram value but which has not already been visited by the typical transition path;

(iii) the algorithm stops if it reaches the box containing the target state $\mathbf{X}_{B}$.

In addition, the full typical path estimation algorithm uses a self-correcting method to avoid dead ends when there are no valid neighbours to be the next point in the trajectory. The spirit of the path-finding algorithm is similar to the depth-first search algorithm [Cormen et al., 2009]. We found that, as long as the histogram is not fragmented, i.e., there is a sufficient number of accumulated trajectories or large enough histogram bins, the algorithm converges. Possible artefacts created by this estimation include spiralling near the initial equilibrium (because of the concentric shell structure of the local probability density function (4.2.10) and zigzagging at the histogram box size. They can be both addressed by a clean-up algorithm: starting from the first box, at each box $\mathbf{X}_{j}$, if the trajectory goes back to one of its neighbours at a later time, with $\mathbf{X}_{j+k}$ being the latest neighbour visit, we erase the points $\mathbf{X}_{j+1}, \ldots, \mathbf{X}_{j+k}$ from the trajectory. Both the path-finding and the clean-up algorithms can be found at https : / github.com/pascalwangt/ PyGMAM.

\subsection{Results}

In this section, we apply both modifications to TAMS (ellipsoids in the score function and typical path estimation) to different problems. 


\subsubsection{Incorporating ellipsoids in the score function}

First, we apply this modified TAMS method to the two-dimensional double-well system defined by (4.2.2). In general, the level sets of the score function do not have the shape of an ellipsoid. Hence, there is no level $\phi^{\text {target }}$ such that the 0.95 confidence ellipsoid $\mathcal{E}$ coincides with $B=\left\{\mathbf{X} \in \mathbb{R}^{n} \mid \phi(\mathbf{X})>\phi^{\text {target }}\right\}$. Here we propose a general method to modify any target score function so that we are able to choose the target set to be exactly the confidence ellipsoid of $\mathbf{X}_{B}$. Let $\mathcal{E}$ be the
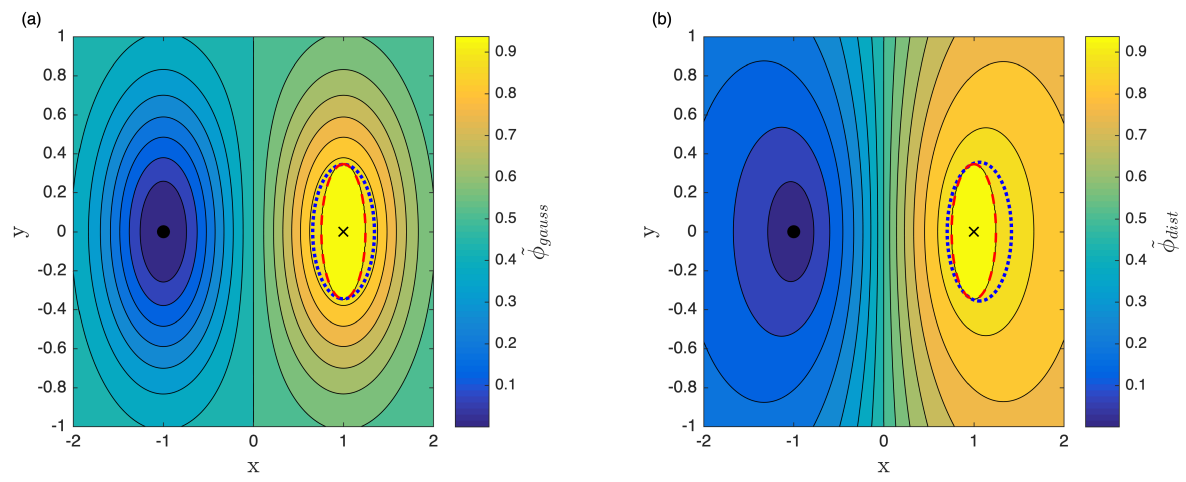

Figure 4.3: (a) Contour levels of the modified score function $\widetilde{\phi}_{\text {gauss }}$, for the two-dimensional double-well system represented in (4.1), according to the procedure described in eq. (4.3.2). The level set $\phi^{\text {target }}$ (dotted blue line) of the former score function $\phi_{\text {gauss }}$ is tangent to the ellipsoid. The level set $\widetilde{\phi}^{\text {target }}$ (dashed red) of the modified score function $\widetilde{\phi}_{\text {gauss }}$ coincides with the ellipsoid. (b) Same plot, for the score function $\widetilde{\phi}_{\text {dist }}$.

0.95-confidence ellipsoid around the equilibrium state $\mathbf{X}_{B}$ and $\phi$ (e.g. $\phi_{\text {gauss }}$ ) the score function to be modified. We first compute the level $\widetilde{\phi}^{\text {target }}$, defined as the minimum of the score function $\phi$ on the confidence ellipsoid $\mathcal{E}$ :

$$
\tilde{\phi}^{\text {target }} \equiv \min _{\mathbf{X} \in \mathcal{E}} \phi(\mathbf{X})
$$

such that the set $\left\{\mathbf{X} \in \mathbb{R}^{n} \mid \phi(\mathbf{X})>\widetilde{\phi}^{\text {target }}\right\}$ contains the ellipsoid $\mathcal{E}$ and is tangent to $\mathcal{E}$. This can be done numerically by generating a mesh of points around $\mathbf{X}_{B}$, then selecting the points inside $\mathcal{E}$ by comparing their norm $\left\|\mathbf{X}-\mathbf{X}_{B}\right\|_{C_{B}^{-1}}^{2}$ with the quantile $Q_{\alpha}$ and finally computing the minimum $\widetilde{\phi}^{\text {target }}$ of $\phi$ on these points. Then, define the modified score function $\tilde{\phi}$ in the following way:

$$
\widetilde{\phi}(\mathbf{X}) \equiv \begin{cases}1 & \text { if } \mathbf{X} \in \mathcal{E} \text { i.e. }\left\|\mathbf{X}-\mathbf{X}_{B}\right\|_{C_{B}^{-1}}^{2}<Q_{\alpha} \\ \tilde{\phi}^{\text {target }} & \text { if } \phi(\mathbf{X})>\widetilde{\phi}^{\text {target }} \text { and } \mathbf{X} \notin \mathcal{E} \\ \phi(\mathbf{X}) & \text { otherwise }\end{cases}
$$


The target set $B=\left\{\mathbf{X} \in \mathbb{R}^{n} \mid \widetilde{\phi}(\mathbf{X})>\widetilde{\phi}^{\text {target }}\right\}$ for the modified score function $\widetilde{\phi}$ turns out to coincide with $\mathcal{E}$. We apply this procedure on both the score functions $\phi_{\text {gauss }}$ and $\phi_{\text {dist }}$ and the results for the improved score functions $\widetilde{\phi}_{\text {dist }}$ and $\widetilde{\phi}_{\text {gauss }}$ are shown in Fig. 4.3.

\subsubsection{Designing a score function based on a typical transition path}

In order to show how to design a score function based on a typical transition path, we consider a two-dimensional system slightly less trivial than the double-well system, i.e. a two-dimensional system with the following potential:

$$
\begin{aligned}
V(x, y) & =\underbrace{0.1 x^{2}+0.05 y^{2}}_{\text {global confinement }}+\underbrace{30 e^{-(x / 2)^{2}}(1+\tanh (15-y))}_{\text {potential barrier at } \mathrm{y}<15, \mathrm{x}=0}+ \\
& -\underbrace{10 e^{((x+6) / 2)^{2}-(y / 2)^{2}}}_{\text {left potential well }}-\underbrace{10 e^{-((x-6) / 2)^{2}-(y / 2)^{2}}}_{\text {right potential well }},
\end{aligned}
$$

depicted in Fig. 4.4(a). It consists in two energy minima at $\mathbf{X}_{A} \approx(-5.77,0)$ and $\mathbf{X}_{B} \approx(5.77,0)$ and a potential barrier spanning $y<15$ and at $x=0$. The dynamics is then given by the SDE (4.2.1), with drift $F\left(\mathbf{X}_{t}\right)=-\nabla V\left(\mathbf{X}_{t}\right)$.

The dynamics of the system is quite interesting, as it exhibits two distinct regimes for transition paths, depending on the noise level $\sigma$ (assuming $G=\sigma I$ ). At high noise $\left(\sigma^{2} \gg \Delta V\right.$, i.e. the potential barrier height), trajectories are likely to cross the potential barrier. At low noise $\left(\sigma^{2} \ll \Delta V\right)$, trajectories are not likely to cross the barrier and the trajectories which undergo the transition instead go through the upper channel at $y \sim 15$. Typical examples of such trajectories are shown in Fig. 4.4(b). In a recent paper, Rolland and Simonnet [2015] investigated the convergence properties of another rare event algorithm using a triplewell potential system, which also exhibits two regimes of preferred transition paths depending on the noise. They found a strong dependency of the statistics of the algorithm (e.g., the number of iterations) and the duration of reactive trajectories on the choice of score function. We expect the same behaviour when applying TAMS to this system. We also hope that this system reveals differences in performance between various score functions.

Fig. 4.5(a) shows an histogram computed with 300 transition paths for the system with the potential given by eq. (4.3.3), with noise level set at $\sigma=3$. On top of it, a typical transition path was estimated using the algorithm sketched in the previous section. As already mentioned, some artefacts created by the estimation (such as spiraling or zigzagging) can be corrected using a clean-up algorithm. The result is shown in Fig. 4.5(b): the empirical estimation of the typical path is a very reasonable approximation of the typical transition path. In fact, it resembles the instanton around which the transition paths are clustered at lower noise 

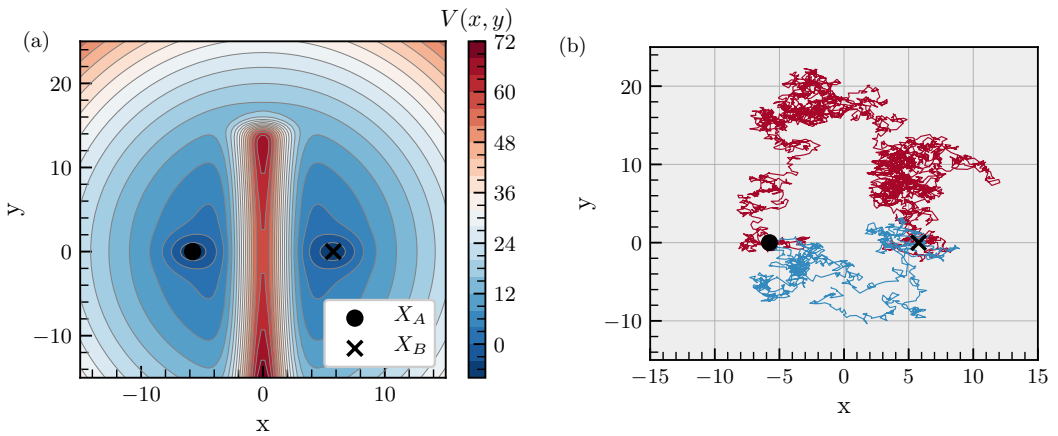

Figure 4.4: (a) Potential landscape of the two-dimensional gradient system defined by eq. (4.3.3). Two energy minima are located at $\mathbf{X}_{A} \approx(-5.77,0)$ and $\mathbf{X}_{B} \approx(5.77,0)$. They are separated by a potential barrier spanning $y<15$ and at $x=0$. (b) Typical transition paths from $\mathbf{X}_{A}$ to $\mathbf{X}_{B}$ for two noise levels $\sigma$. At high noise, trajectories can cross the potential barrier ( $\sigma=10$, blue). At low noise, trajectories go through the upper channel ( $\sigma=3$, red).

(Fig. 4.5(b)). The instanton was calculated by implementing the geometric minimum action method [Heymann and Vanden-Eijnden, 2008].

Note that, if instead we accumulated trajectories at high noise $\sigma>10$, we would obtain trajectories going from $\mathbf{X}_{A}$ to $\mathbf{X}_{B}$ in a straight line, which is the typical path at high noise similar to [Rolland and Simonnet, 2015]. This typical transition path is substantially different from the instanton, which goes through the upper channel. Thus, our method can be advantageous in multistable systems where the typical path depends on the noise level. We can start at high noise and reapply the empirical estimation of the typical path each time the noise level is decreased.

Given a typical transition path $\mathcal{C}$, we present the design of a score function $\phi_{\mathcal{C}}$ which encourages trajectories to follow the transition path $\mathcal{C}$ such that it gives a reasonable approximation of the static committor. Let us consider a trajectory $\mathcal{C}(s)$ in $\mathbb{R}^{n}$ parametrised by arclength $s \in[0,1]$. Then we define the score function $\phi_{\mathcal{C}}$, called path-based score function, such that it grows from 0 to 1 along the trajectory from $\mathbf{X}_{A}$ to $\mathbf{X}_{B}$ and decays exponentially along the direction transverse to the trajectory:

$$
\begin{aligned}
\phi_{\mathcal{C}}: \mathbb{R}^{n} & \rightarrow[0,1] \\
\mathbf{X} & \mapsto s(\mathbf{X}, \mathcal{C}) \times \exp \left(-\frac{d(\mathbf{X}, \mathcal{C})^{2}}{d_{0}^{2}}\right)
\end{aligned}
$$

where $d(\mathbf{X}, \mathcal{C})=\inf _{s \in[0,1]}\|\mathbf{X}-\mathcal{C}(s)\|_{d}$ is the distance between $\mathbf{X}$ and the trajectory $\mathcal{C}(s), s(\mathbf{X}, \mathcal{C})$ is the curvilinear coordinate of the position on the trajectory that satisfies the infimum in the definition of $d(\mathbf{X}, \mathcal{C})$ and $d_{0}$ is the characteristic decay length (free parameter). The so-defined score function $\phi_{\mathcal{C}}$ is shown in Fig. 4.6 for 

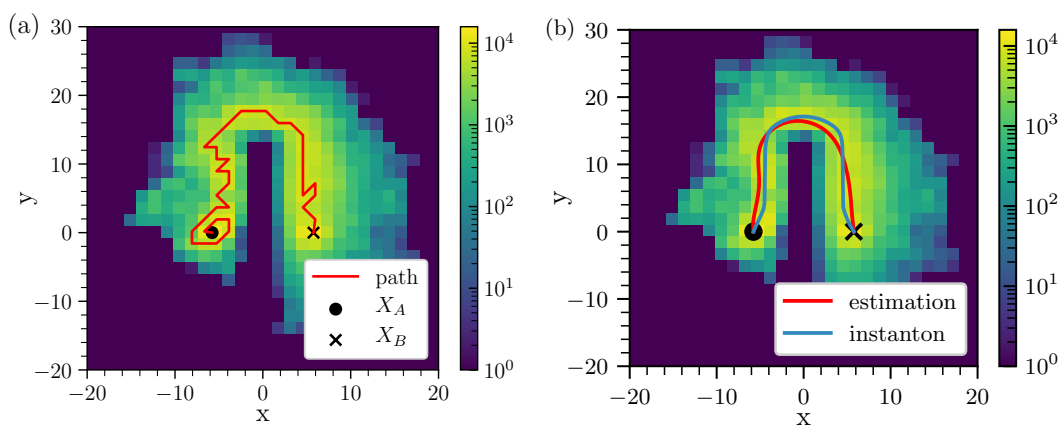

Figure 4.5: (a) Histogram of $N=300$ transition paths at noise level $\sigma=3$, using the modified score function $\widetilde{\phi}_{\text {gauss }}$ with $\beta=1.5$, defined by eq. (4.2.6) and implemented with (4.3.2). The corresponding transition probability $\left(p=2 \times 10^{-3}\right)$ is high enough so that Direct Monte Carlo sampling could have been used to produce a similar histogram. The bin resolution $(\Delta x=1.4, \Delta y=1.75)$ is coarse for illustration purposes. The histogram is used as input in the path-finding algorithm which produces the transition path in red. Gridscale spiralling occurs near the initial state $\mathbf{X}_{A}$ because of the concentric shell structure of the local probability density function given by eq. (4.2.10). (b) Same histogram as the left panel. The estimated typical transition path (red) has been cleaned up from its grid-scale spiraling and zigzags with the clean-up algorithm and has then been smoothed. It strongly resembles the instanton (blue path), which was computed by implementing the geometric action minimum method [Heymann and Vanden-Eijnden, 2008].

the estimated transition path $\mathcal{C}$ shown in Fig. 4.5 and two values of decay length $d_{0}=20$ and $d_{0}=200$.
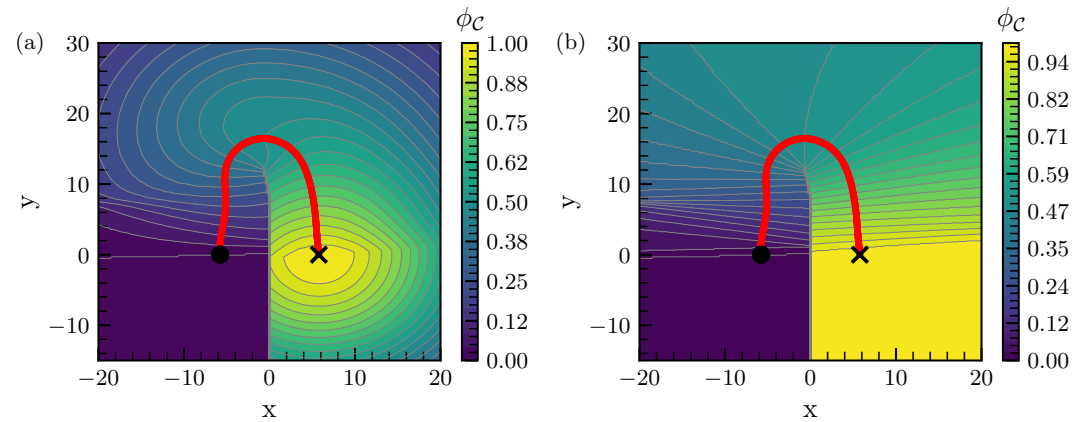

Figure 4.6: (a) Contour levels of the score function $\phi_{\mathcal{C}}$ defined in eq. (4.3.4), associated with the estimated transition path $\mathcal{C}$ shown in Fig. 4.5 and with decay length $d_{0}=20$. (b) Same figure for $d_{0}=200$.

The score function increases from 0 to 1 along the trajectory. Thus, it encodes the preferred direction that the system has to follow. This contrasts with the generic 
score functions $\phi_{\text {gauss }}$ and $\phi_{\text {dist }}$ which are symmetrical in $y$ : they do not contain the information that the system has to increase in $y$ in order to make the transition to $\mathbf{X}_{B}$. Note that the method we developed here can be applied, in principle, to systems of any dimension. As shown in Fig. 4.6(b) the score function $\phi_{\mathcal{C}}$ is discontinuous because the trajectory has positive curvature. The discontinuity is located near the axis $x=0$. Indeed, when crossing the axis $x=0$, the closest point on $\mathcal{C}$ changes and $s(\mathbf{X}, \mathcal{C})$ is discontinuous.

Next, we applied TAMS with the path-based score function $\phi_{\mathcal{C}}$ to the twodimensional double well with a potential wall. We compare its performance with the previously defined score functions $\phi_{\text {dist }}$ and $\phi_{\text {gauss }}$. In fact, we use the associated modified score functions, such that the target set $B$ matches the 0.95 confidence ellipsoid (we drop the tildes for readability). We use the following parameters:

\begin{tabular}{c|c|c} 
& Parameter & Value \\
\hline$T$ & duration of a trajectory & 20 \\
$d t$ & time step & 0.01 \\
$\phi^{\text {target }}$ & target score & chosen to match the 0.95 -confidence ellipsoid
\end{tabular}

Table 4.1: Parameters for TAMS algorithm with the improved score function

We show in Fig. 4.7(a) the transition probability estimates using the score functions $\phi_{\text {dist }}, \phi_{\text {gauss }}$ (with $\beta=1.5$ ) and $\phi_{\mathcal{C}}$ (with decay parameters $d_{0}=2,20,200$ ) averaged over 10 instances of the algorithm. The probability estimates are in good agreement between each other and with a Monte Carlo estimation for $\sigma>2.5$. The score function $\phi_{\mathcal{C}}$ is robust with respect to the choice of the decay length $d_{0}$.

The performances of the numerical methods are next measured using the worknormalised relative error $\epsilon$ which combines the variance of the algorithm and its computational cost [Glynn et al., 2009]:

$$
\epsilon \equiv \frac{\sigma_{\hat{p}}}{\mu_{\hat{p}}} \times \omega,
$$

where $\mu_{\hat{p}}$ and $\sigma_{\hat{p}}$ are the mean and standard deviation of the probability estimate over the different instances, $\omega$ is the average number of time steps calculated in one realisation. In short, $\epsilon$ measures how precise the numerical method is at equal computational cost. The smaller $\epsilon$, the better the algorithm performs.

The results are shown in Fig. 4.7(b). In this system, the score functions $\phi_{\text {dist }}$, $\phi_{\text {gauss }}$ and $\phi_{\mathcal{C}}$ have little difference in performance. For the lowest noise values, the path-based score function has at most a 30\% smaller error $\epsilon$ than the score function $\phi_{\text {gauss }}$. Changing the decay length $d_{0}$ hardly changes the error $\epsilon$. When adjusting the parameters of the potential $V$ or applying the same method to the triple well system used in [Rolland and Simonnet, 2015], the performance gain, while being 
often present, never systematically exceeded 30\%. All in all, in this category of twodimensional systems, using the path-based score function approach yields little improvement.
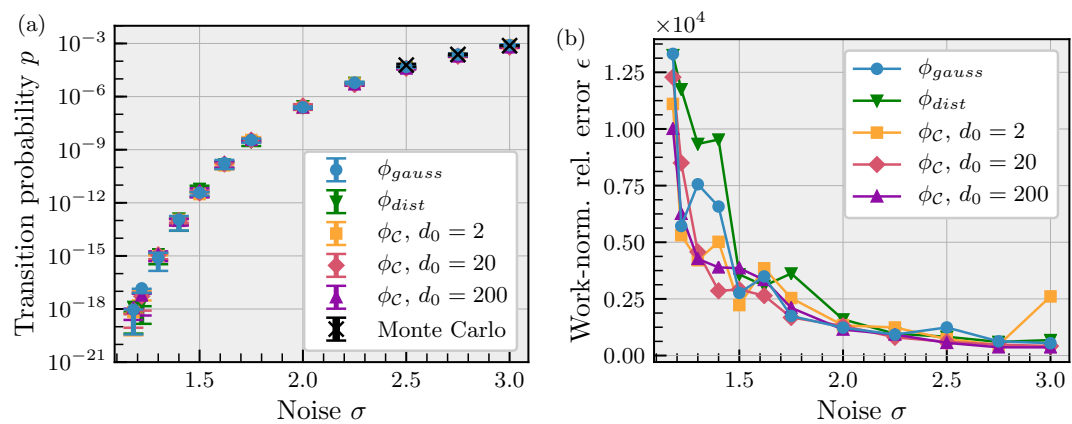

Figure 4.7: (a) Transition probability $p$ as a function of noise parameter $\sigma$. Mean estimates and interquartile range (error bars) over $N_{\text {samples }}=10$ independent realisations of the TAMS algorithm using the score function $\phi_{\text {dist }}$ (green down triangle) $\phi_{\text {gauss }}$ (with $\beta=1.5$, blue circle) and $\phi_{\mathcal{C}}$ with decay parameters $d_{0}=2,20,200$ (yellow square, pink diamond, purple up triangle) are shown. The Monte Carlo estimate (black cross) has been run with $N=$ $5 \times 10^{5}$ trajectories with target set the confidence ellipsoids around $\mathbf{X}_{B}$, with associated standard deviation $\sigma_{p}=\sqrt{p(1-p) / N}$ (error bars). There is an overall good agreement between the numerical estimations. The path-based score function is robust to the choice of decay parameter. (b) Performance of the score functions measured by the work-normalised error $\epsilon$ as a function of noise $\sigma$ (same markers as left panel). At most, there is a $30 \%$ decrease in error when using the path-based score function $\phi_{\mathcal{C}}$ at low noise. Note that at high noise $\sigma=3$, using a short decay length $d_{0}=2$ with $\phi_{\mathcal{C}}$ leads to poor performance because the greater values of $\phi_{\mathcal{C}}$ are tightly concentrated around the estimated instanton whereas typical transition paths are not necessarily clustered around it. Otherwise, the performance of the score functions are roughly similar.

\subsubsection{Transition probabilities in a box model of the AMOC}

Finally, as a main application of one of the techniques shown in this chapter, we consider the system defined in section 3.1, which represents a box model of the Atlantic Meridional Ocean Circulation (AMOC). The system consists of five stochastic differential equations, plus one algebraic constraint, representing the salt conservation in the model (see eq. (3.1.1)):

$$
\begin{aligned}
\mathrm{d} \mathbf{Y}_{t} & =F_{1}\left(\mathbf{Y}_{t}, Z_{t}\right) \mathrm{d} t+B d \mathbf{W}_{t}, \\
0 & =F_{2}\left(\mathbf{Y}_{t}, Z_{t}\right) .
\end{aligned}
$$

In the equations above, we split the state of the system $\mathbf{X}_{t}$ into two parts: $\mathbf{Y}_{t}$, which includes four of the salinities of box model, plus $D$, i.e. the depth of the 
pycnocline, and $Z_{t}$, which represents the salinity of the deep box $\left(S_{d}\right)$. As the noise is applied only on the asymmetric component of the atmospheric freshwater flux $\left(E_{a}\right)$, it directly affects only two of the variables $S_{n}$ and $S_{s}$ ). Moreover, the stochastic increments associated with the two variables are identical, to make sure that each decrease of freshwater forcing in the southern box results in the same increase of it in the northern box in the model. As a result, the noise is not spatially independent and the noise matrix $G$ is no longer diagonal: it consists of a $(5 \times 1)$ row vector, with only two elements different from zero. For a reasonable choice of the parameters, the deterministic system is in a bistable regime, which means that there are two possible equilibrium states, under the same forcing conditions. In general, we are interested in studying transitions between the present-day AMOC $\left(\mathbf{X}_{A}\right)$ and the collapsed state $\left(\mathbf{X}_{B}\right)$. See the next chapter for a full description of the model and the stability of its equilibria.

For a differential-algebraic system of equations (DAEs), such as the system in eq. (4.3.6), we need to be particularly careful while computing the covariance ellipsoids. First of all, we make use of the Schur complement of the Jacobian of the system, which allows to calculate the covariance matrix when an algebraic constraint is present [Baars et al., 2017]. Nevertheless, the resulting matrix $C_{B}$ is singular, with two eigenvalues being equal to zero. One of the corresponding eigenvectors is a vector pointing in the direction of the variable $D$ (depth of the pycnocline). The reason behind it is that the differential equation governing the evolution of $D$ does not contain any of the other variables (see eq. (3.1.1)), when the system is in the 'off' state $\left(\mathbf{X}_{B}\right)$. This results in $D$ not being affected by the noise, as this is imposed only on two of the salinities. Motivated by this fact, we compute the covariance matrix relative to the salinities $C_{B}^{S}$, removing one degree of freedom from the original matrix. Unfortunately, such matrix still gives one zero eigenvalue, which means that the system does not oscillate around the direction indicated by the corresponding eigenvector: this is due to the salinity conservation (the algebraic equation in the system (4.3.6)).

To overcome this problem, we compute the Moore-Penrose inverse (or pseudoinverse) of the covariance matrix, $C_{B}^{S+}$, by performing a singular value decomposition of $C_{B}^{S}$ and removing the zero eigenvalue, together with the corresponding eigenvector [Ben-Israel and Greville, 2003]. A two-dimensional projection of the ellipsoid constructed for the box model is shown in Fig. 4.8.

For the system (4.3.6), the modified score function is more complicated, as the covariance matrix used to construct the ellipsoid contains only the degrees of freedom related to the salinities of the model, leaving the variable $D$ (depth of the pycnocline) out. From a geometric point of view, that means that the covariance ellipsoid around $\mathbf{X}_{B}$ is degenerated along the $D$-direction. Fig. 4.8 shows a projection of the ellipsoid - once the noise amplitude is fixed - on the plane identified by the variables $S_{n}$ and $S_{s}$ (respectively, the salinity of the northern box and the one of the southern box in the model). The projection was obtained calculating the conditional covariance matrix of the two variables into consideration, given that the other variables are set on their mean value [Wasserman, 2013]. The two- 
dimensional ellipse contains the large majority of the projected time points (on the same plane) of a trajectory that wanders around the equilibrium. By construction, the confidence level of the confinement is higher than the one prescribed for the full dimensional ellipsoid (in this case 0.95). Clearly the shape of the level sets of the score function do not coincide with the one of the ellipsoid. Hence, the importance of modifying the score function appears evident.

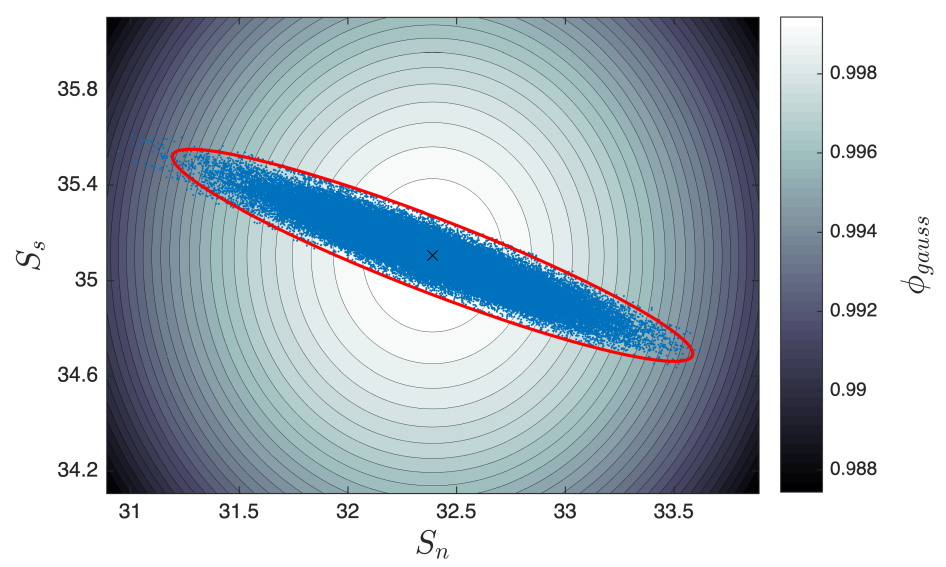

Figure 4.8: Level sets of the score function $\phi_{\text {gauss }}$, in proximity of the AMOC 'off' equilibrium state $\mathbf{X}_{B}$ of the system (3.1.1), projected on the plane identified by the variables $S_{n}$ and $S_{s}$ (respectively, the salinity of the northern box and the one of the southern box in the model). On top of them a trajectory of the system, initiated in the 'off' state, has been projected on the same plane (blue circles). The red ellipse is the two-dimensional projection of the covariance ellipsoid constructed by using the matrix $C_{B}^{S}$ and a confidence level of 0.95 . 98\% of the points composing the trajectory turn out to be inside the ellipsoid.

When constructing an improved score function, in order to evaluate if a state belongs to the neighbourhood of $\mathbf{X}_{B}$, we need to check two conditions: (i) whether the state of the system is inside the salinity covariance ellipsoid drawn around the destination equilibrium, and (ii) whether the variable $D$ of the state is the same as the one of $\mathbf{X}_{B}$. Hence, the improved score function for the box model reads

$$
\widetilde{\phi}_{\text {box }}(\mathbf{X}) \equiv \begin{cases}1 & \text { if } \mathbf{X}^{S} \in \mathcal{E}^{S} \text { and } \mathbf{X}^{D}=\mathbf{X}_{B}^{D} \\ \widetilde{\phi}_{\text {target }} & \text { if } \phi_{\text {gauss }}(\mathbf{X})>\widetilde{\phi}^{\text {target }} \text { and }\left(\mathbf{X}^{S} \notin \mathcal{E}^{S} \text { or } \mathbf{X}^{D} \neq \mathbf{X}_{B}^{D}\right) \\ \phi_{\text {gauss }}(\mathbf{X}) & \text { otherwise }\end{cases}
$$

where $\mathbf{X}^{S}$ indicates the part of the state vector representing the set of the salinities, whereas $X^{D}$ represents the variable $D$. As already mentioned, to check whether a certain state belongs to the salinity ellipsoid, we made use of the pseudo-inverse of $C_{B}^{S}$ in the definition (4.2.12). 
To be able to assess the relevance of a proper definition of the target set in the TAMS algorithm, and hence the importance of using the improved version of the score function, we computed transition probabilities of the AMOC from the presentclimate state to the collapsed state, for reasonable values of the atmospheric forcing and noise. In particular, we chose $\bar{E}_{a}=0.20 S v$ and $f_{\sigma}=0.16$. This last value represents the ratio between the standard deviation of the noise in the atmospheric forcing and its mean value (as already discussed in section 3.1.3). The number of trajectories used in the algorithm was set to 100 , and the time scale at which the probabilities were evaluated was chosen to be 1000 and 10000 years, respectively. For each transition probability, we used three different versions of the algorithm, based on three different settings for the score function. The first two versions were implemented using $\phi_{\text {gauss }}$ (equation (4.2.6)), with two different choices for the threshold $\phi^{\text {target }}$. In the third version, we used $\widetilde{\phi}_{\text {box }}$, where the starting score function was $\phi_{\text {gauss }}$. Each probability was calculated running 15 instances of the algorithm, and then computing the median and the interquartile range (IQR). The results are shown in the Table 4.2 below.

\begin{tabular}{c|c|c} 
Score function & $p$ at 1000 years [IQR] & $p$ at 10000 years [IQR] \\
\hline$\phi_{\text {gauss }}, \phi^{\text {target }}=1-10^{-4}$ & $<10^{-9}$ & $<10^{-9}$ \\
$\phi_{\text {gauss }}, \phi^{\text {target }}=1-10^{-2}$ & $(1.1[0.6: 1.2]) \times 10^{-3}$ & $(5.3[4.7: 6.0]) \times 10^{-2}$ \\
$\widetilde{\phi}_{\text {box }}$ & $<10^{-9}$ & $(2.0[0.7: 3.0]) \times 10^{-3}$
\end{tabular}

Table 4.2: Results of the transition probabilities for the AMOC model using different score functions

\subsection{Summary and discussion}

In this chapter, we presented and applied several improvements to the TAMS rareevent algorithm, when used to compute transitions in multistable systems. The first improvement has to do with a rigorous criterion to define noise-induced transitions. We used confidence ellipsoids $\mathcal{E}$ to formalise this criterion. In turn, this led to the rigorous choice of the target set $B=\mathcal{E}_{B}$ which was traditionally set by arbitrary thresholds. We then showed how to incorporate this definition of $B$ into the score function $\phi$. For certain classes of systems, like the ones containing algebraic constraints in addition to differential equations, or when the noise does not affect one or more directions in the variable space (i.e. the associated covariance matrix is singular), this method requires some precautions. In particular, for the AMOC box model studied in this thesis, we had to adapt the definition of the improved score function (4.3.7), as well as calculate the pseudo-inverse matrix of the covariance matrix, in order to compute the ellipsoid. 
When running TAMS to compute transition probabilities between two states of the AMOC in the system, with different versions of the score function, we found a considerable discrepancy between the obtained values (see Table 4.2). In particular, it appears that setting a very high threshold in the Gaussian score function makes the algorithm detect no transitions (we set up the algorithm so that it stops when the probabilities involved are smaller than $10^{-9}$ ): the reason behind this is that, because of the presence of the noise, we don't expect the state of the system to stay indefinitely close to the destination equilibrium, but rather to wander around it. Therefore, the score function, which assigns the maximum score only to a very small neighbourhood of the equilibrium, is not able to properly recognise transitions. Moreover, it is not surprising that, when using $\phi_{\text {gauss }}$ with a smaller value of $\phi^{\text {target }}(0.99)$ or $\widetilde{\phi}_{b o x}$, we obtain different results: as the shape of the covariance ellipsoid is not spherical (see Fig. 4.8), we expect $\phi_{\text {gauss }}$ to detect transitions even though the state is actually still far from the destination equilibrium, at least in certain directions. As a general rule, we expect $\phi_{\text {gauss }}$ to give incorrect results as long as the ellipsoid of the system is not spherical, regardless of the choice of the threshold $\phi^{\text {target }}$.

This method, while being quite general, is subject to some limitations. While the modified score function $\phi$ is continuous outside $B$, it is constant in the domain $M=\left\{\mathrm{X} \in \mathbb{R}^{n} \mid \phi(\mathrm{X})>\widetilde{\phi}^{\text {target }}\right\} \backslash \mathcal{E}$ (see Fig. 4.3(b)). This means that in the TAMS algorithm, branching will never occur inside $M$, but at the boundary $\partial M$. This can have an influence on the convergence of TAMS if the level sets of the initial score function $\phi$ have a pathological shape near $\mathbf{X}_{B}$ and the spatial extension of $D$ is not negligible. Nevertheless, we expect this to have little impact because this phenomenon is localised near the target state $\mathbf{X}_{B}$. Therefore, the trajectories will naturally converge towards $\mathbf{X}_{B}$ as a result of the dynamics, even without the help of the branching process of TAMS.

However, to ensure that the confidence ellipsoid $\mathcal{E}$ defines a meaningful target set, one needs to be sure that $\mathcal{E}$ is contained inside the basin of attraction of the target state $\mathbf{X}_{B}$. While this is the case in the limit of small noise $\sigma \rightarrow 0$, it might not be the case for finite noise. A solution to this issue would be to compute the basin of attraction $\mathcal{V}$ of $\mathbf{X}_{B}$ and define the target set $B$ as the intersection $B=\mathcal{E} \cap \mathcal{V}$. However, we reasonably expect that in the generic case, this occurs when the noise level $\sigma$ is high enough so that transitions are less rare and a direct Monte Carlo estimation is sufficient to estimate transition probabilities.

Next, we proposed a systematic method of defining a score function, designed to approximate the static committor, based on empirical transition paths. We proposed an algorithm to estimate the typical transition path under a high-noise level, which is then used to define a family of score functions with a single decay parameter $d_{0}$.

We applied our method to a two-dimensional well with a potential barrier. We found that our typical path estimation gave satisfactory results and that the associated score function, while discontinuous, remained unbiased and relatively 
insensitive to the change of decay parameter $d_{0}$. While we did not find significant performance improvements over existing non-trivial score functions, we think that differences will become apparent if applied to higher dimensional systems, where there are more directions to fluctuate in.

One key limitation of our approach of constructing the path-based score function $\phi_{\mathcal{C}}$ is the computer memory needed to store the trajectory histogram, which becomes prohibitively huge for high-dimensional systems such as discretised partial differential equation (PDE) systems. As an example, a 50×50 two-dimensional grid storing 4 variables in each cell (e.g. two velocity components, pressure and a tracer) with 10 bins of resolution in each degree of freedom would require more than 10 Petabytes of memory, which is unreasonable. However, this limitation can be easily bypassed by defining the objects needed to run the TAMS algorithm, namely the score function $\phi$ and the target set $B$, in a reduced space of much fewer dimensions. For instance, Bouchet et al. [2019] studied the dynamics of the barotropic beta-plane quasi-geostrophic equations describing Jupiter's turbulent atmosphere. While the PDE system was evolved in the full phase space, their rare-event algorithm was run in a reduced 3-dimensional phase space defined by three Fourier coefficients. The target set $B$ and the score function were defined on this reduced space. Moreover, they accumulated transition trajectories in a 3-dimensional histogram and showed their concentration around instantons. By applying the path-finding algorithm, an empirical estimation of the instanton can be made. This offers a viable alternative to solving a minimization problem in the full space to compute the instanton and then project it in the reduced space, which is next to intractable for this system.

Another way to define the reduced space $\mathcal{V}$ in which to run TAMS is to consider the principal components, also called empirical orthogonal functions (EOF), which are the eigenvectors of the covariance matrix. One idea, suggested by Baars et al. [2019], is to retain the principal components $\left\{\mathbf{Y}_{1}^{A}, \ldots, \mathbf{Y}_{k}^{A}, \mathbf{Y}_{1}^{B}, \ldots, \mathbf{Y}_{k^{\prime}}^{B}\right\}$ with largest variance (i.e. eigenvalue) of the covariance matrix at the initial state $\mathbf{X}_{A}$ and target state $\mathbf{X}_{B}$. Principal components represent the directions in which the system fluctuates the most. They are then assumed to be the directions which capture best the noise-driven dynamics. When studying transitions in a two-dimensional primitive equation model of the Atlantic Meridional Overturning Circulation [Toom et al., 2011, Baars, 2019] projected the dynamics in a reduced space $\mathcal{W} \equiv \operatorname{Span}\left\{\mathbf{X}_{A}, \mathbf{X}_{B}, \mathbf{Y}_{1}^{A}\right.$, $\left.\ldots, \mathbf{Y}_{k}^{A}, \mathbf{Y}_{1}^{B}, \ldots, \mathbf{Y}_{k^{\prime}}^{B}\right\}$ of dimension ( 500) still too large to apply the histogram method directly. However, one idea is that the TAMS algorithm could be run in an even smaller space $\mathcal{V} \equiv \operatorname{Span}\left\{\mathbf{X}_{A}, \mathbf{X}_{B}, \mathbf{Y}_{1}^{A}, \ldots, \mathbf{Y}_{d}^{A}, \mathbf{Y}_{1}^{B}, \ldots, \mathbf{Y}_{d^{\prime}}^{B}\right\}$ (of dimension <10), while still computing the dynamics in the space $\mathcal{W}$. Then, the memory required to store a histogram becomes reasonable and our histogram method can be applied.

Another potential issue of our modified TAMS method is the fact that the score function $\phi_{\mathcal{C}}$ is discontinuous because the trajectory has positive curvature, as shown in Fig. 4.6(b). The discontinuity is located near the axis $x=0$. Indeed, when crossing the axis $x=0$, the closest point on $\mathcal{C}$ changes and $s(\mathbf{X}, \mathcal{C})$ is discontinuous. In fact, in the mathematical proofs about the statistical and convergence properties 
of the probability estimator [Cérou et al., 2016], the score functions are assumed to be continuous. Nevertheless, in our applications, we did not detect any statistically significant bias in the probability estimator due to the discontinuity. Moreover, some meaning can be attributed to the discontinuity: it is located at the boundary between the attraction basins of $\mathbf{X}_{A}$ and $\mathbf{X}_{B}$ and it thus reflects a qualitative change of behaviour in the system. Crossing this boundary means that the trajectory converges to $\mathbf{X}_{B}$ instead of $\mathbf{X}_{A}$, if $\sigma=0$. In addition, the remnant of a discontinuity is observed in the static committor of the similar triple well system used in [Rolland and Simonnet, 2015]. Indeed, their Figure 4(c) shows the contour plots of the static committor in the low noise regime. A steep gradient is present at the $x=0$ boundary, which gives further evidence that the discontinuity of $\phi_{\mathcal{C}}$ may not be problematic.

Further testing of the ideas presented in this chapter in high-dimensional systems such as discretised PDEs would give more insight as to the effectiveness of our approach, compared to more generic score functions used up to now. Moreover, incorporating some form of time-dependence in the score function $\phi$ to specifically optimise TAMS would constitute an interesting project. 



\section{Chapter 5}

\section{Transition probabilities of noise-induced transitions of the Atlantic Ocean Circulation}

In this chapter, we determine estimates of the transition probability of noise-induced transitions of the AMOC, within a certain time period, using the TAMS algorithm. We find that there are two types of transitions, with a partial or full collapse of the AMOC, having different transition probabilities. For the present-day state, we estimate the transition probability of the partial collapse over the next 100 years to be about $15 \%$, with a high sensitivity of this probability to the surface freshwater noise amplitude.

This chapter is based on the following publication:

Castellana, D., Baars, S., Wubs, F. W., and Dijkstra, H. A. (2019). Transition probabilities of noise-induced transitions of the Atlantic Ocean Circulation, Scientific Reports, 9(1), 1-7. 


\subsection{Introduction}

In the first chapter we described the role of the Atlantic Meridional Overturning Circulation in the climate system and discussed the stability of its present-day state.

In chapter 3 , we introduced $M_{o v}$, namely the freshwater transport carried by the overturning circulation at the southern boundary of the Atlantic Ocean, and described its role as an indicator for the stability of the AMOC [de Vries and Weber, 2005]. A negative (positive) value of $M_{o v}$ indicates that the AMOC is in a multiple (single) equilibria regime. $M_{o v}$ was developed from ocean-only model results and hence neglects the effects of atmospheric feedbacks [Gent, 2018]. However, it has been widely used in models to interpret the behaviour of the AMOC [Cimatoribus et al., 2014, Mecking et al., 2016]. Present-day observational results show that $M_{o v}$ is in the range of $-0.35 \mathrm{~Sv}$ to $-0.1 \mathrm{~Sv}$ [Weijer et al., 2001, Huisman et al., 2010, Bryden et al., 2011, Liu et al., 2014]. Hence, if $M_{o v}$ is indeed a proper indicator, then the present-day AMOC is in a multiple equilibria regime.

Our aim is to determine the probability of a transition to a collapsed state within a certain period of time (e.g. into the future), for example due to noise in the surface freshwater forcing. By using the TAMS algorithm, we present results for the transition probabilities of noise-induced changes in the AMOC. As such technique is still computationally demanding, we apply it here to a detailed box model of the AMOC (presented in chapter 3), in which the value of $M_{o v}$ is a precise measure of the multiple equilibria regime [Cimatoribus et al., 2014]. Thanks to $M_{o v}$, we can make an estimate, from the model results, of the transition probabilities for the observed present-day AMOC. In section 5.2 we summarise the main features of the box model, which was already described in chapter 3 . Section 5.3 shows the results, with the respect to the bifurcation diagrams of the deterministic system and the application of the TAMS algorithm when additive white noise is added to the system. A summary and discussion of the results conclude the chapter.

\subsection{Model and methods}

The model used in this chapter was already introduced in chapter 3 and its graphical representation can be found in Fig. 3.1. Here, we summarise its main features. The Atlantic Ocean is modelled as five boxes, which interact though salinity fluxes. The downwelling in the North Atlantic (and hence the AMOC strength) is represented by the term $q_{N}$, while $q_{S}$ is the difference between the wind-driven Ekman flow $\left(q_{E k}\right)$ and the eddy-induced flow $\left(q_{e}\right)$, the latter associated with baroclinic instabilities of the Antarctic Circumpolar Current. The upwelling volume transport from the deep layer to the pycnocline layer is represented by $q_{U}$. Two coefficients, $r_{N}$ and $r_{S}$, capture the effect of the wind-driven subtropical gyres on the salinity transport. Finally, the Atlantic Ocean circulation model is forced by external freshwater fluxes, split into a symmetric $\left(E_{s}\right)$ and an asymmetric $\left(E_{a}\right)$ component; the noise will only be incorporated through $E_{a}$. 
The model in [Cimatoribus et al., 2014] has been extended to allow for the existence of both a strong and weak AMOC state, which we refer to below as the 'on' and 'off' state, respectively. The present-day state of a strong AMOC is represented by values $q_{N}>q_{S}>q_{U}>0$. The 'off' AMOC state occurs only when the downwelling in the Northern Atlantic stops $\left(q_{N}=0\right)$ and the circulation in the South Atlantic is reversed $\left(q_{s}<0\right)$.

The model is particularly suitable for investigating the variability of the AMOC due to the buoyancy anomalies. While capturing the essential physical processes involved in the dynamics of the AMOC (e.g. the wind-induced upwelling in the Southern Ocean), it gives emphasis to the salt-advection feedback, which is the feedback responsible for the collapse of the AMOC. In the real ocean, wind-induced variability can lead to the occurrence of extremes in the strength of the AMOC, as found in the RAPID measurements [Zhao and Johns, 2014]. These effects are not captured by our model. Nevertheless, this does not affect the capabilities of the model to capture the dominant processes underlying the buoyancy-induced variability of the AMOC. Recently, [Wood et al., 2019] also showed that a 5-box dynamical model can be calibrated with GCM output to capture (within some error bars) the critical behaviour of the AMOC. In conclusion, the model used here, forced by the interannual time scale noise in the freshwater flux, is fit for purpose to look at freshwater-caused transitions (in a bistable AMOC regime) which are affected by the salt-advection feedback.

Transition probabilities between states in this model are computed using the Trajectory-Adaptive Multilevel Sampling (TAMS) method [Lestang et al., 2018].

\subsection{Results}

Variations in the forcing will only be considered through the asymmetric freshwater flux, which is written as

$$
E_{a}(t)=\bar{E}_{a}+E_{a}^{\sigma}(t)
$$

Here the deterministic value $\bar{E}_{a}$ is constant and the stochastic part $\left(E_{a}^{\sigma}(t)=\sigma \zeta(t)\right)$ is represented by a zero mean, unit variance white noise process $\zeta(t)$ and a standard deviation $\sigma$.

Steady states of the deterministic model $(\sigma=0)$ for standard values of the parameters (see table 3.1) are shown in Fig. 5.1. Two saddle-node bifurcation points bound the multiple equilibria regime: for each value of $\bar{E}_{a}$ in the range $[0.06,0.35]$, both 'on' and 'off' AMOC states exist (Fig. 5.1). The pycnocline depth is much shallower (up to $1000 \mathrm{~m}$ ) for the 'on' AMOC state (Fig. 5.1(a)) than for the 'off' AMOC state. The main difference between the diagrams shown in Fig. 5.1 and the ones that can be found in [Cimatoribus et al., 2014] is the presence of the 'off' AMOC state, since the model allows solutions with $q_{N}=0$ (Fig. 5.1(b)) and $q_{S}<0$ (Fig. 5.1(c)). The freshwater transport at $30^{\circ} \mathrm{S}$ carried by the AMOC is 
given by

$$
M_{o v}=-\frac{q_{S}}{S_{0}}\left(S_{t s}-S_{d}\right)
$$

As already shown in [Cimatoribus et al., 2014], the sign of $M_{o v}$ is an adequate indicator for the multiple equilibria regime (Fig. 5.1(d)). The bifurcation diagrams of the salinities in the different boxes and the upwelling strength $q_{U}$ are shown in Fig. 5.1: It appears that the 'off' AMOC state is characterised by a constant upwelling from the deep ocean.

We next fix the deterministic part of the asymmetric freshwater forcing $\left(\bar{E}_{a}\right)$ to a value within the multiple equilibria regime and add stochastic forcing $(\sigma>0)$. The value of $\sigma$ is chosen as a percentage of the deterministic forcing, hence $\sigma=$ $f_{\sigma} \bar{E}_{a}$, based on observational data from $P-E$ time series in the Atlantic ocean, assuming that the noise represents interannual time scale variability [Ganopolski and Rahmstorf, 2002]. We estimated a lower bound of this amplitude $f_{\sigma}$ to be 0.1 (see section 3.1.3).

In Fig. 5.3 an example of a model trajectory is shown, which is initialised at the 'on' AMOC equilibrium state (with $\bar{E}_{a}=0.29 \mathrm{~Sv}$ and $M_{o v}=-0.21 \mathrm{~Sv}$ ). The applied noise in the freshwater forcing initially affects the polar boxes, and then propagates through the rest of the basin. The salinity in the southern box $S_{s}$ (Fig. 5.3(a)) switches between the two equilibrium values (see Fig. 5.2), while the variability in the salinity of the northern box $S_{n}$ (Fig. 5.3(b)) is not strong enough to reach the value corresponding to the 'off' AMOC state. Moreover, the depth of the pycnocline $D$ (Fig. 5.3(c)) remains essentially constant throughout the whole simulation ( $D \sim 900 \mathrm{~m}$ in comparison to the $1700 \mathrm{~m}$ necessary to reach the value of the 'off' AMOC state). The most frequent abrupt changes involve the downwelling $q_{N}$, which repeatedly decreases to zero throughout the duration of the simulation (Fig. 5.3(d)). However, the AMOC does not seem to undergo a full transition to the 'off' state, as shown by the behaviour of the other variables.

These results are very instructive on the behaviour of the system, as they indicate that the AMOC does not necessarily collapse (reach the 'off' state), even if the salinity in the boxes show quite some variability. In fact, the fast variations in the freshwater input determine large changes in the salinity of the polar boxes, to the point where the downwelling collapses. However, such collapse would have to be sustained for a long time to be able to considerably affect the deep ocean circulation. For this reason, we consider two kinds of transitions in the model: an F-type (fast) transition with a temporary cessation of the downwelling, and an S-type (slow) transition to an 'off' state. As already suggested by the model results in Fig. 5.3, probabilities for the two types of transitions substantially differ in magnitude.

Depending on the transition event one wants to study, the TAMS method has to be properly implemented, in order to select for the trajectories that undergo the transition. That means that a proper score function needs to be designed for each of the two transitions. 
(a)

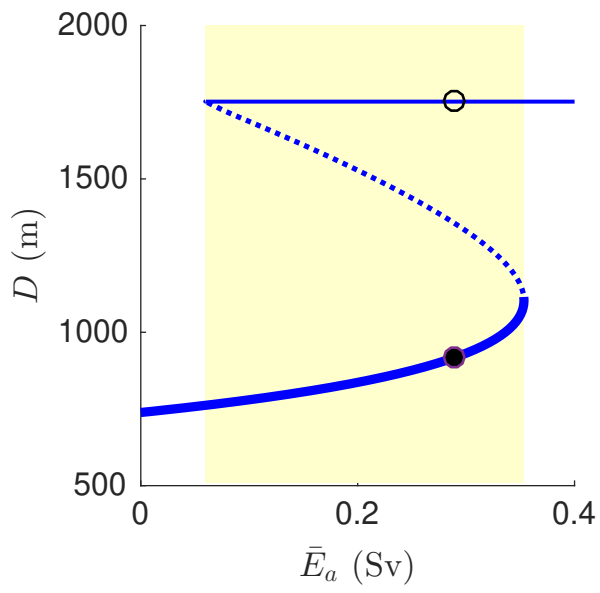

(c)

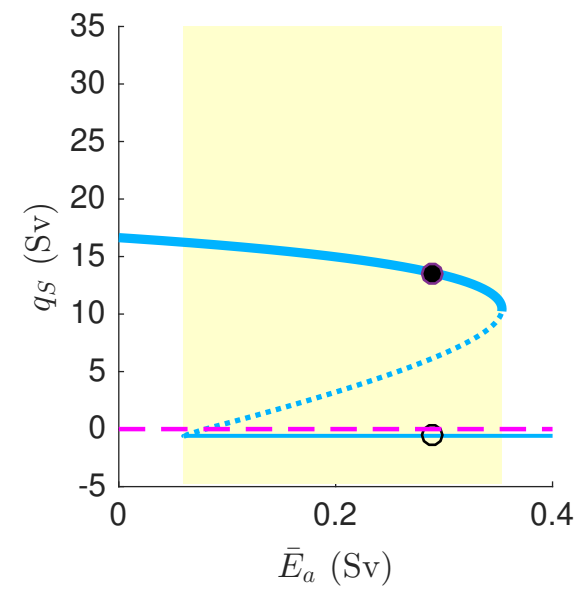

(b)

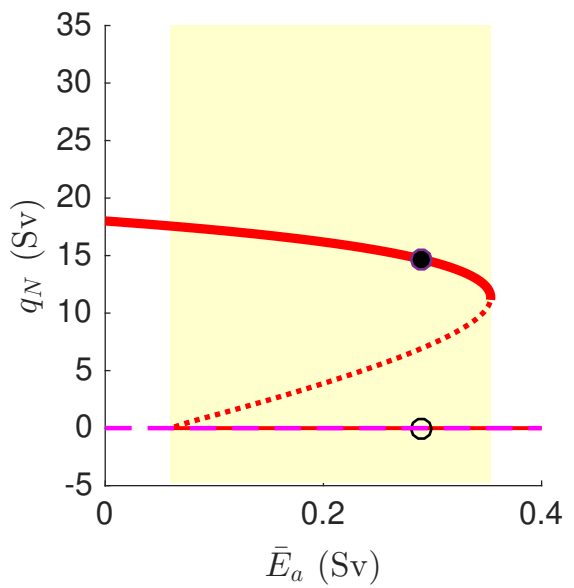

(d)

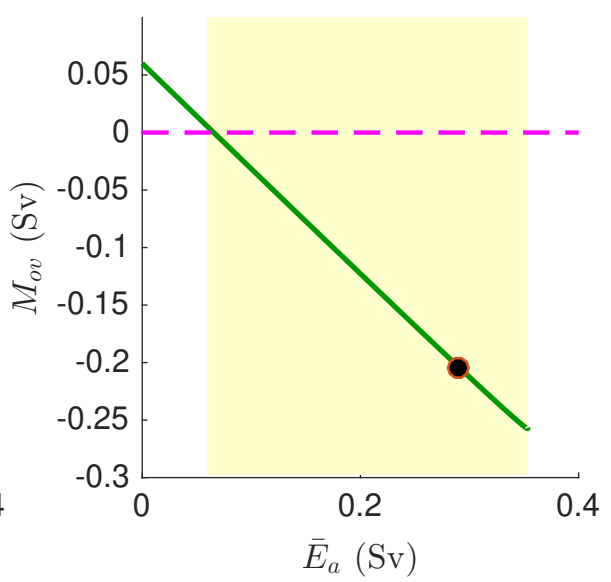

Figure 5.1: (a) Bifurcation diagram for the depth of the pycnocline versus the parameter $\bar{E}_{a}$. Solid (dashed) lines indicate stable (unstable) equilibria of the system. Among the solid lines, the thicker ones represent the AMOC 'on' state, while the thinner ones correspond to the 'off' state. The yellow area indicates the bistable regime. The black circles indicate the value of $\bar{E}_{a}$ chosen for the time simulation in Fig. 5.3. The starting (destination) equilibrium belongs to the 'on' ('off') branch of the diagram. (b-c) The same diagrams, respectively for the downwelling in the North Atlantic $\left(q_{N}\right)$ and the upwelling in the Southern Ocean $\left(q_{S}\right)$. The dashed magenta lines indicate null transport: in the collapsed state, the downwelling stops, while the circulation through the basin is reversed, as indicated by the negative value of $q_{S}$. (d) $M_{o v}$, calculated following eq. (5.3.2), as a function of the asymmetric freshwater forcing $\bar{E}_{a}$. The indicator is calculated only for the 'on' state of the system. $M_{o v}$ is positive (negative) in the monostable (bistable) regime of the AMOC. 

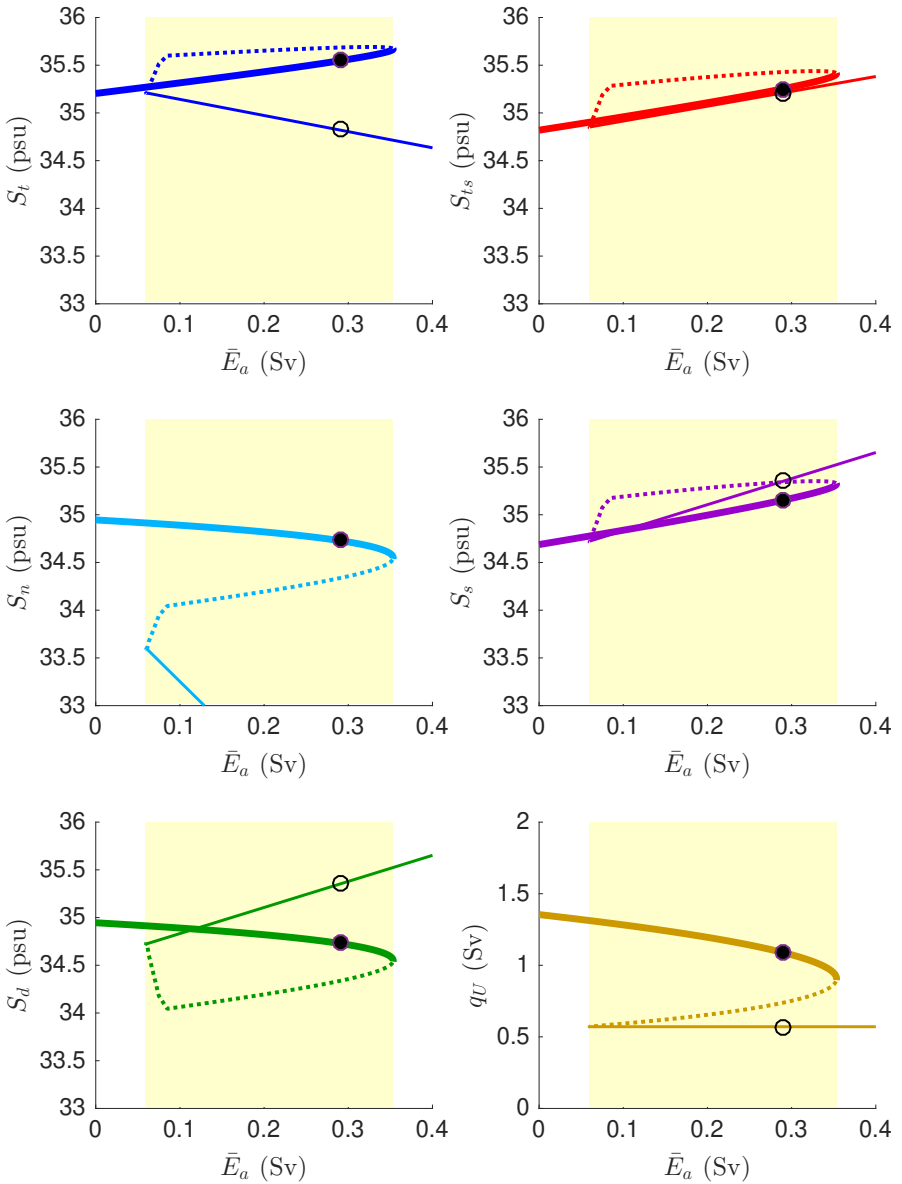

Figure 5.2: Bifurcation diagrams for the salinity variables of the model and for the diffusive upwelling $\left(q_{U}\right)$ with respect to the deterministic parameter $\bar{E}_{a}$. Solid (dashed) lines indicate stable (unstable) equilibria of the system. Among the solid lines, the thicker ones represent the so called AMOC 'on' state, while the thinner ones correspond to the collapsed 'off' state. The yellow area indicates the bistable regime. The black circles indicate the value of $\bar{E}_{a}$ chosen for the time simulation in Fig. 5.3. The starting (destination) equilibrium belongs to the 'on' ('off') branch of the diagram. 
(a)

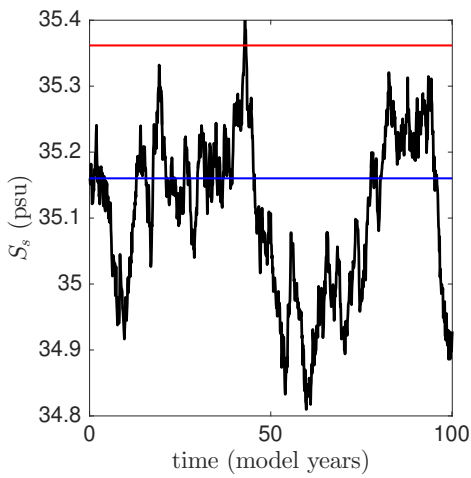

(c)

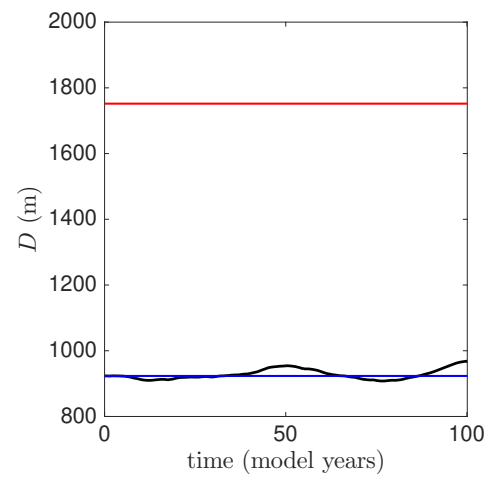

(b)

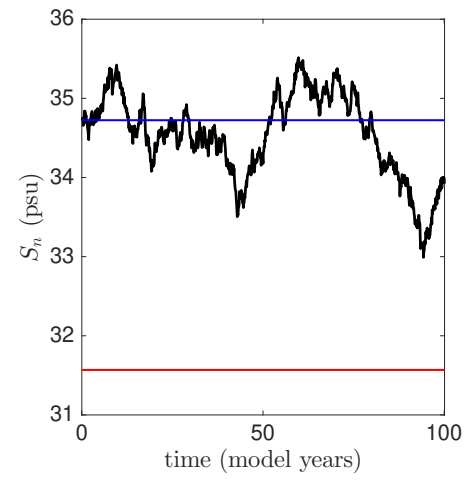

(d)

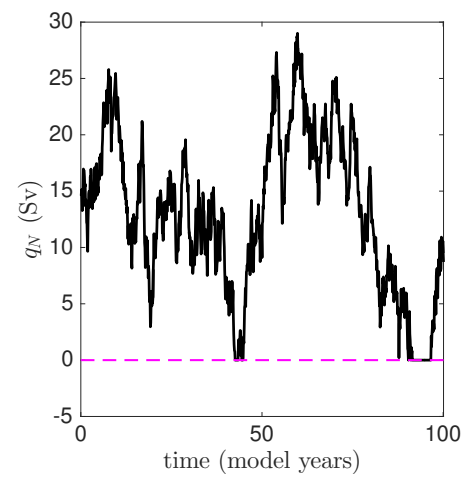

Figure 5.3: Trajectory of the box model, with reference parameters as in table (3.1), and with $\bar{E}_{a}=0.29 \mathrm{~Sv}$ and $f_{\sigma}=0.16$. The corresponding value of $M_{o v}$ is $-0.21 \mathrm{~Sv}$. The initial condition is centered on the 'on' state of the system. Plots are shown for (a) $S_{s}$, (b) $S_{n}$, (c) $D$ and (d) $q_{N}$. The blue (red) line indicates the 'on' ('off') steady state, for the chosen parameters. The dashed magenta line indicates a zero transport. 
An F-type transition does not constitute an actual transition between different steady state, as the ones defined in chapter 3 , but rather the occurrence of an extreme event in the system. That means that we are looking at the extreme behaviour or one variable, namely the downwelling $\left(q_{N}\right)$, and the score function can be defined a linear function of the $q_{N}$, which assumes the value 0 when the system is in the 'on' state and 1 when $q_{N}=0$. Hence

$$
\phi_{F}(\mathbf{x})=1-\frac{q_{N}(\mathbf{x})}{q_{N}\left(\mathbf{x}_{o n}\right)},
$$

where $\mathbf{x}$ represents the state vector of the system, $\mathbf{x}_{\text {on }}$ the 'on' state, and $q_{N}(\mathbf{x})$ the value of the downwelling.

For an S-type transition, i.e., a full transition to the 'off' state, the score function is taken as the improved Gaussian score function defined in chapter 4 (see section 4.3.3). The improvement was made by using the covariance ellipsoid computed in the 'off' state as the target set for the score function.

To compute the transition probabilities for a range of possible values of freshwater forcing for F-type transitions, we ran 15 instances of the TAMS algorithm for a grid of size $100 \times 125$ in the $\left(\bar{E}_{a}, \sigma\right)$ space. Because we can connect $\bar{E}_{a}$ and $M_{o v}$ through the steady state (Fig. 5.1(d)), and the noise is a fraction $f_{\sigma}$ of the steady state value $\bar{E}_{a}$, we plot (Fig. 5.4(a)) the transition probabilities, using a transition time interval of 100 years, in the space $\left(M_{o v}, f_{\sigma}\right)$.

The range of parameters, which corresponds to the present-day climate conditions, is the region bounded by $f_{\sigma}>0.1$ and $-0.35 \mathrm{~Sv}<M_{o v}<-0.1 \mathrm{~Sv}$, taking the values at $24^{\circ} \mathrm{S}$ as representative for $M_{o v}$ [Bryden et al., 2011]. The range of transition probabilities is then quite broad: depending on the actual value of $M_{o v}$ and the stochastic forcing, an F-type transition can be very unlikely to occur (bottom-left area of the plot), or near certain (top-right area). Hence, we show the transition probability as a function of $f_{\sigma}$ for $M_{o v}=-0.1 \mathrm{~Sv}$ and $M_{o v}=-0.2 \mathrm{~Sv}$ in Fig. 5.4(b) and (c), respectively. For $M_{o v}=-0.1 \mathrm{~Sv}$ the transition probabilities only become non-negligible for $f_{\sigma}>0.2$, but for $M_{o v}=-0.2 \mathrm{~Sv}$ (which could be seen as a mean value over the available estimates), the transition probability is already about $15 \%$ at $f_{\sigma}=0.1$.

In order to test the robustness of our results, we computed transition probabilities for slightly different versions of our model, characterised by different values of certain parameters. In all the simulations, we kept the value of $M_{o v}$ and the noise constant. Note that, as the configuration of the system varies, a certain value of $M_{o v}$ does not correspond to a unique value of $\bar{E}_{a}$. Based on the results of the analysis, shown in Fig. 5.5, we can conclude that the method seems to be robust under uncertainties in the estimation of the parameters, in particular transport by the southern subtropical gyre $r_{S}$, vertical diffusivity $\kappa$ and eddy diffusivity $A_{G M}$ (for which reference values were shown in table 3.1).

When determining the S-type transition probability of the AMOC to the 'off' state we obtain very low probabilities $\left(<10^{-9}\right)$ within 100 years, regardless of the 

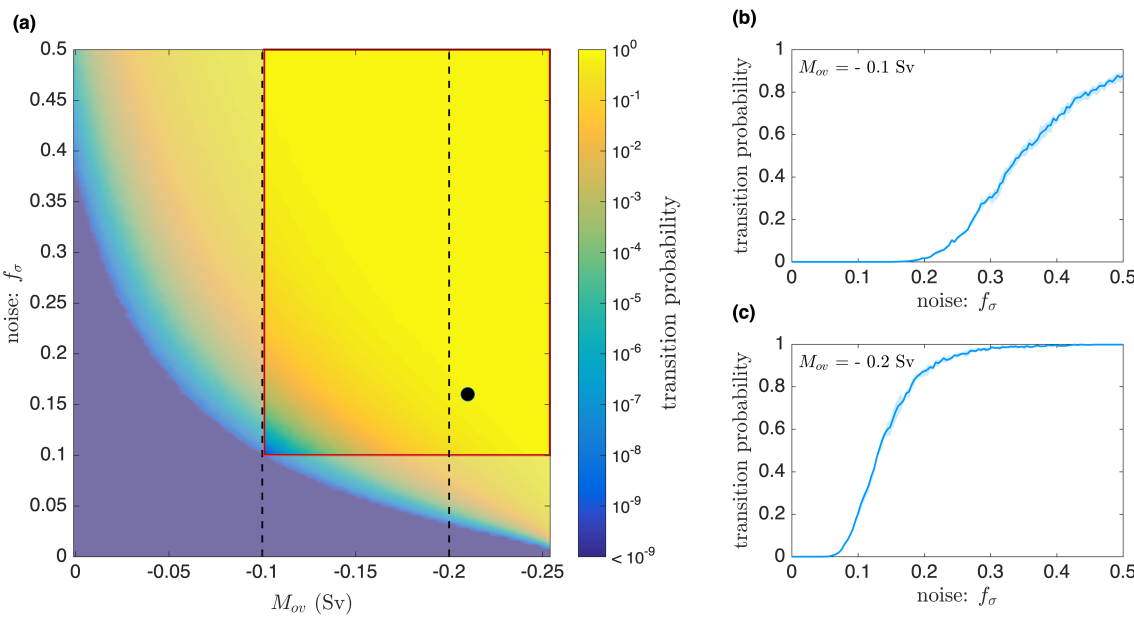

Figure 5.4: (a) Transition probabilities of the F-type transition (cessation of the downwelling) in 100 years, calculated for each couple of the parameters $\left(M_{o v}, f_{\sigma}\right)$, chosen on a $100 \times 125$ grid. The computation was done with the TAMS algorithm, which was repeated 15 times for each transition probability computation. In this way, the error bars (not shown) turn out to be quite narrow (see panels (b) and (c) as an example). The area that corresponds to the range of parameters that can best represent the present-day climate is highlighted in the red box. It is bounded by the minimum amplitude of noise found in observations (0.1), and the observed range of values of $M_{o v}(-0.35$ to $-0.1 \mathrm{~Sv})$. The black circle indicates the parameters chosen for the time simulation in Fig. 5.3. (b-c) Transition probabilities as a function of $f_{\sigma}$ for two particular choices of $M_{o v}$ as indicated by the dashed lines in (a). The shaded areas represent the interquartile range for the probabilities. Notice the steepness of the gradient with respect to the value of the noise, which increases with more negative values of $M_{o v}$. 
Chapter 5. Transition probabilities of noise-induced transitions of the

Atlantic Ocean Circulation

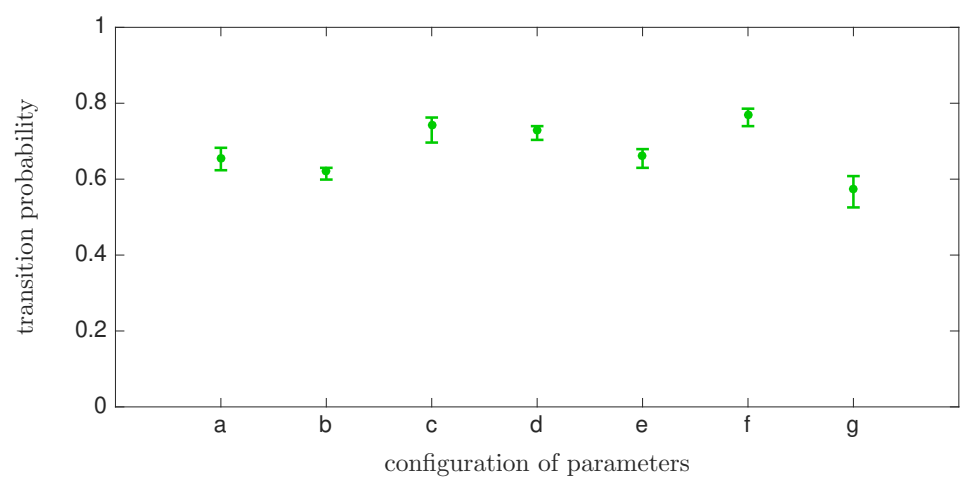

Figure 5.5: Transition probabilities calculated for $T=100$ years, $M_{o v}=-0.18 \mathrm{~Sv}$ and $f_{\sigma}=$ 0.18 , for different choices of the parameters $r_{S}$ (transport by the southern subtropical gyre), $\kappa$ (vertical diffusivity) and $A_{G M}$ (eddy diffusivity). For each value of the probability, the bar represents the interquartile range, calculated from 10 different repetitions of the TAMS algorithm. The configuration (a) is the standard one, according to the parameters in table 3.1. Configurations (b) and (c) are characterised by a different value of $r_{S}$, respectively 1.25 $\times 10^{7}$ and $0.75 \times 10^{7} \mathrm{~m}^{3} \mathrm{~s}^{-1}( \pm 25 \%)$. In configurations (d) and (e), $\kappa$ was set respectively to $2 \times 10^{-5}$ and $0.5 \times 10^{-5} \mathrm{~m}^{2} \mathrm{~s}^{-1}$ (2 times and half the value of the standard configuration). Finally, in configurations (f) and (g) $A_{G M}$ was set respectively to 1870 and $1530 \mathrm{~m}^{2} \mathrm{~s}^{-1}$ ( \pm $10 \%)$.

values of the parameters of the model. If we increase the time interval, we find that only for time scales of the order of 10,000 years, the transition probabilities become non-negligible (as shown in section 4.3.3). An example of the evolution of the system on long time scales, showing an S-type transition, is shown in Fig. 5.6.

\subsection{Summary and discussion}

In this chapter we investigated the probability of noise-induced transitions of the AMOC to a collapsed state within a specific time period, using a conceptual boxmodel representation of the AMOC circulation [Cimatoribus et al., 2014]. Thanks to its simplicity, the model is suitable for the application of the Trajectory-Adaptive Multilevel Splitting (TAMS) algorithm. At the same time, it is quite comprehensive in terms of physical processes driving the circulation and allows to calculate the freshwater transported by the AMOC at $30^{\circ} \mathrm{S}\left(M_{o v}\right)$, which connects the results of our model to state-of-art climate models and observations. In the model, the sign of $M_{o v}$ represents a perfect indicator for the multiple equilibria regime (Fig. 5.1(d)), in line with what has been found in more detailed numerical models [de Vries and Weber, 2005, Huisman et al., 2010, Hawkins et al., 2011].

The analysis of the time evolution of the stochastic model shows that two kinds of transitions occur, namely a cessation of the downwelling in the North Atlantic 
(a)

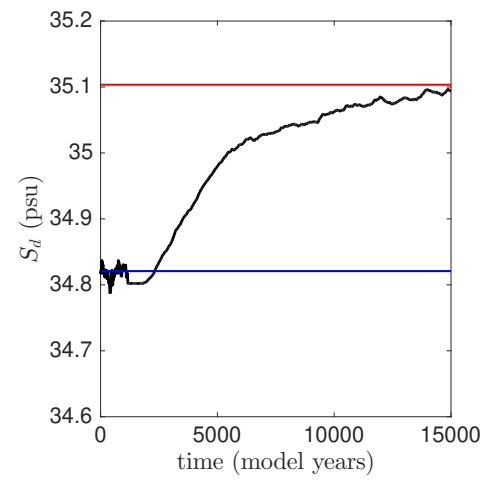

(c)

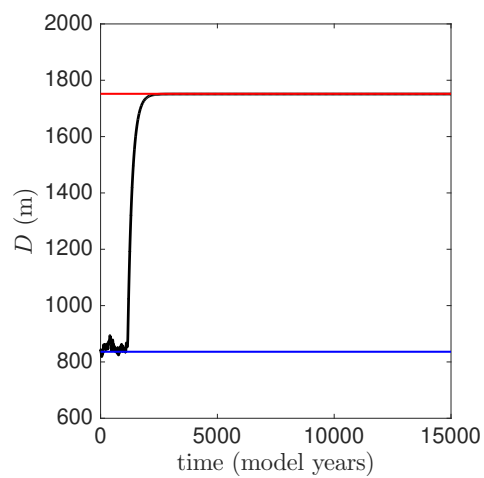

(b)

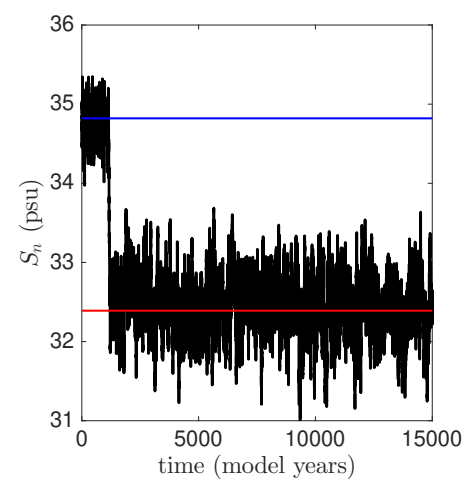

(d)

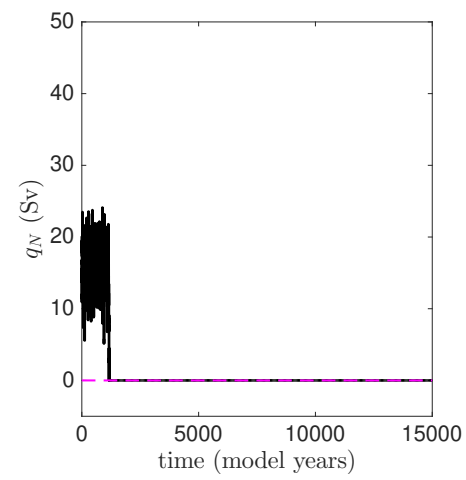

Figure 5.6: Trajectory of the box model on a long time scale (15,000 years), with reference parameters as in Table (3.1) and with $\bar{E}_{a}=0.20 \mathrm{~Sv}$ and $f_{\sigma}=0.16$. The corresponding value of $M_{o v}$ is $-0.12 \mathrm{~Sv}$. The initial condition is centered on the 'on' state of the system. Plots are shown for (a) $S_{d}$, (b) $S_{n}$, (c) $D$ and (d) $q_{N}$. The blue (red) line indicates the 'on' ('off') state, for the chosen parameters. The plot (d) shows the evolution of the downwelling in the North Atlantic. The dashed magenta line indicates null transport. The figure clearly shows that the different quantities reach the values corresponding to the collapsed state at different times. The system undergoes a full transition at the end of the time frame considered here, as illustrated by the value of the salinity of the deep box, the slowest variable, which eventually reaches the value corresponding to the 'off' state. The downwelling had already stopped after about 1000 years, indicating that an F-type transition had occurred much earlier. 

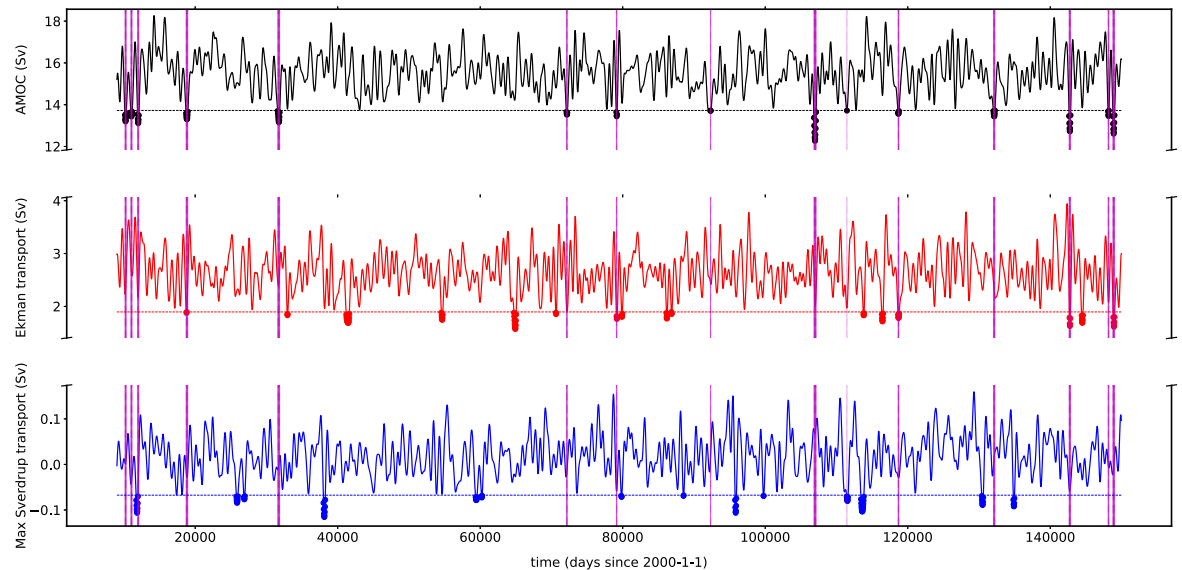

Figure 5.7: 20 month Butterworth low-pass filtered time series of the maximum value of the AMOC at $26.5^{\circ} \mathrm{N}$ (black), the zonally integrated Ekman transport (red) and the maximum Sverdrup transport at the same latitude (blue), in the pre-industrial control simulation of the CMIP5 model MIROC5. The dashed horizontal lines indicate, for each time series, the lower bound of the 2 standard deviations confidence interval around the mean. The circles identify the time points at which extreme events occur, defined as the values extending below the confidence interval. The dotted magenta vertical lines are drawn in correspondence with the extreme events of the AMOC on top of all the time series. In this way, it is possible to assess whether a certain event is related with anomalies in the wind circulation. Some of the events seem to occur independently of changes in both Ekman and Sverdrup transports (e.g. the one found between time 80000 and 100000).

(called F-type transition) and a full transition to a collapsed state (called S-type transition). We found that, while an F-type transition can have very high transition probabilities over a period of 100 years under a reasonable choice of the parameters, the S-type transition seems unlikely to occur over this period, regardless of the values of the parameters involved. As expected, the probability of Ftype transitions increases with decreasing $M_{o v}$ (more negative) and with increasing noise amplitude $f_{\sigma}$ (see Fig. 5.4(a)). For the area in the parameter space by which the present-day climate is best represented, our results indicate that the probability of a (temporary) cessation of the downwelling is almost certain for values of $M_{o v}<-0.2 \mathrm{~Sv}$.

Indeed, such transitions may have been found already in the RAPID program measurements [Cunningham et al., 2007, Smeed et al., 2014]: the dips in the time series of the AMOC strength at $26.5^{\circ} \mathrm{N}$ (up to negative values) suggest the occurrence of extreme events in the circulation which are not directly connected to any subsequent collapse of the whole circulation system. They may be induced either by noise in the freshwater flux, in the heat flux or in the wind-stress field. The same phenomenon can be found in most of the CMIP5 models, where the 
time series of the AMOC at $26.5^{\circ} \mathrm{N}$ shows several dips during control simulations (Fig. 5.7). The comparison between the occurrence of extremes in the transports associated with wind anomalies and dips in the AMOC strength suggests that the role of the stochastic buoyancy forcing can lead to extreme events in the AMOC. Indeed, while some extreme events in the AMOC can be attributed to anomalies in the wind field, others seem to occur independently of those anomalies. Therefore, we believe that anomalies in the freshwater forcing are responsible for (F-type) transitions in the AMOC.

In the context of paleoclimate transitions, such as the Dansgaard-Oeschger events, it is interesting that S-type transitions, involving a full-scale transition to an 'off' state, have only non-negligible transition probabilities in our model on multimillennial time scales (see Table 4.2). However, to connect our model results to these events, a change in the background climate state (glacial) is necessary and possibly also a slow variation of the noise and the freshwater forcing should be introduced in the model, which is outside the scope of this study.

\section{Acknowledgements}

We acknowledge the World Climate Research Programme's Working Group on Coupled Modelling, which is responsible for CMIP, and we thank the climate modelling group MIROC (Atmosphere and Ocean Research Institute - The University of Tokyo, National Institute for Environmental Studies, and Japan Agency for Marine-Earth Science and Technology), for producing and making available their model output. For CMIP the U.S. Department of Energy's Program for Climate Model Diagnosis and Intercomparison provides coordinating support and led development of software infrastructure in partnership with the Global Organization for Earth System Science Portals. 



\section{Chapter 6}

\section{Noise-induced transitions of the Atlantic Meridional Overturning Circulation in CMIP5 models}

By studying transition probabilities of the Atlantic Meridional Overturning Circulation (AMOC) in an ensemble of CMIP5 climate models, we revisit one of the stability indicators of the AMOC, i.e. the freshwater transport carried by the AMOC at the southern boundary of the Atlantic basin. A correction to this indicator, based on the transition probabilities, is suggested to measure whether an AMOC state is in a multiple equilibrium regime or not. As a consequence, the AMOC of all CMIP5 models considered is in a multiple equilibrium regime and hence, in principle, a collapsed AMOC state should exist in each of these models. The results further demonstrate the dependence of the Atlantic surface freshwater flux on the AMOC and the impact of extreme events in the AMOC on temperatures in the North Atlantic region.

This chapter is based on the following manuscript:

Castellana, D. and Dijkstra, H. A. (2020). Noise-induced Transitions of the Atlantic Meridional Overturning Circulation in CMIP5 models. Manuscript under review. 
Chapter 6. Noise-induced transitions of the Atlantic Meridional Overturning Circulation in CMIP5 models

\subsection{Introduction}

The Atlantic Meridional Overturning Circulation (AMOC) plays a crucial role in the climate system, as it transports about 1.5 PW of heat northward in the Atlantic Ocean. Observations from the RAPID project have shown evidence of a decrease of a few Sverdrups (Sv) in the strength of the AMOC over the period 2004-2017 [Smeed et al., 2019]. It is well known [Stommel, 1961, Bryan, 1986, Cheng et al., 2013] that the AMOC strength is sensitive to the surface freshwater forcing. In particular, model results [Cessi and Young, 1992, Weijer et al., 2001, Rahmstorf et al., 2005, Hawkins et al., 2011] indicate that the AMOC can have more than one stable state under the same surface forcing conditions. The physics behind the multistability of the AMOC is well understood and due to the salt-advection feedback [Stommel, 1961].

Projections from state-of-the-art climate models from the IPCC (Intergovernmental Panel on Climate Change, 2013) indicate that the AMOC will weaken under the effect of the anthropogenic climate change at a rate up to about $1 \mathrm{~Sv}$ per decade [Smeed et al., 2018]. When studying the output of climate models which are part of the CMIP5 (Coupled Model Intercomparison Project Phase 5) [Taylor et al., 2012], the stability of the AMOC cannot be determined easily because of the long simulation times required [Gent, 2018]. This is one of the reasons why multiple equilibria have not been found in such models. Another reason might be that the AMOC states are not in a multiple equilibrium regime, possibly due to biases in the models [Drijfhout et al., 2011]. Anyway, it cannot be excluded that the AMOC in the present-day climate is in a multiple equilibrium regime [Weijer et al., 2019].

As already discussed, to determine whether a multiple equilibrium regime exists in a model, an indicator of the AMOC stability has been developed: $M_{o v}$. However, such indicator was only developed in the framework of ocean-only models, as it ignores feedbacks between ocean and atmosphere [Drijfhout et al., 2011, Gent, 2018].

When the present-day AMOC is in a multiple equilibrium regime, changes in the freshwater forcing may induce a transition to a weak or 'collapsed' AMOC state. When such a transition is not induced by the crossing of a critical boundary, but by fast variability in the forcing ('noise'), it is called a noise-induced transition [Ashwin et al., 2012]. In the previous chapter, such noise-induced transitions were studied in a box model of the Atlantic Ocean, where $M_{o v}$ is a perfect indicator for the multiple equilibrium regime of the AMOC. It was found that two types of noiseinduced transitions can occur: a full collapse of the AMOC, referred to as S-type transition, and a partial and temporary collapse, associated with a temporary cessation of the downwelling in the North Atlantic, referred to as F-type transition. While an S-type transition is unlikely to occur within the next 100 years, the likelihood of an F-type transition strongly depends on the amplitude of the freshwater noise and the value of $M_{o v}$.

It is important to investigate the noise-induced transitions of the AMOC in 
CMIP5 models as these transitions can have substantial consequences for the climate of the North Atlantic. Several studies have shown the impact of the AMOC variability on the sea surface temperature (SST) of the North Atlantic Ocean [Latif et al., 2004, Kloewer et al., 2014], as well as its influence on the European climate [Palter, 2015, Drijfhout, 2015]. Roberts et al. [2013] computed regression maps of changes in SST, associated with a change in the AMOC, using decadal mean time series from an ensemble of CMIP5 models, and found positive correlations in the North Atlantic. The area that seems to be the most sensitive to the AMOC variability is the Atlantic Subpolar Gyre. Rahmstorf et al. [2015] developed an AMOC index, based on the SST difference between the Atlantic Subpolar Gyre and the Northern Hemisphere, which was found to correlate well with the AMOC strength. Duchez et al. [2016] investigated the link between the observed AMOC anomalies at $26^{\circ} \mathrm{N}$ and satellite based Atlantic SST anomalies and showed that there is a significant correlation between these quantities.

The main aim of this chapter is to analyse noise-induced AMOC transitions in CMIP5 model results. After an overview of the model output and the methodology in section 6.2, we detect F-type transitions in section 6.3 in CMIP5 models and connect these to $M_{o v}$. Moreover, the consequences of noise-induced transitions in the AMOC strength are explored, especially with regard to anomalies in the surface temperatures of the North Atlantic region. A summary and discussion of the results follow in section 6.4 .

\subsection{Models and methods}

In this study, pre-industrial control simulations from 10 different CMIP5 models were analysed and compared (see Table 6.1). Due to the availability of the specific data needed, we were not able to use other CMIP5 models. The choice of using control simulations is motivated by the fact that we are interested in noise-induced transitions of the AMOC, with the background climate state being in equilibrium.

The AMOC strength is represented by the maximum value (over depth) of the meridional overturning stream function at $26.5^{\circ} \mathrm{N}$, from now indicated by $\Psi_{M}$. Mid-latitude AMOC variations are partly induced by wind-stress variations, mainly through the Ekman transport [Zhao and Johns, 2014], which is a relatively fast response as it is accomplished by (barotropic) waves. The noise-induced transitions of the AMOC we are interested in are induced by high-frequency variations in the freshwater forcing (see previous chapter). Because of the nonlinear nature of the ocean circulation, it is generally not possible to separate both AMOC responses to buoyancy and wind-stress variations. Nevertheless, Biastoch et al. [2008] argue that such a separation is meaningful for mid-latitude AMOC variability. Hence, we modify the AMOC time series by subtracting the Ekman transport at $26.5^{\circ} \mathrm{N}$, indicated by $\Psi_{E}$ and expect that variations in the resulting time series $\Psi_{M}^{c o r}=\Psi_{M}-\Psi_{E}$ better reflect those occurring through surface buoyancy fluctuations. Our choice is in agreement with the results shown by Polo et al. [2014], who conclude that buoy- 
Chapter 6. Noise-induced transitions of the Atlantic Meridional Overturning Circulation in CMIP5 models

ancy forcing dominates in the geostrophic transport anomalies $\Psi_{M}^{\text {cor }}$ (i.e., AMOC Ekman transport) on decadal time scales, whereas wind-stress induced fluctuations are mostly confined to intraseasonal and interannual time scales.

To calculate the high-frequency variability of the freshwater flux over the Atlantic Ocean, i.e. the main component of the 'noise', we follow the same approach as in chapter 3 (see section 3.1.3). First, the integrated freshwater flux over the northern Atlantic region $\left(50^{\circ} \mathrm{N}-70^{\circ} \mathrm{N}\right)$, indicated by $F_{S}^{N}$, and a southern region (south of $40^{\circ} S$ ), indicate by $F_{S}^{S}$, is calculated. Next, the difference $F_{S}^{N S}=F_{S}^{N}-F_{S}^{S}$ is determined and the 'noise' used here is the yearly-averaged component of $F_{S}^{N S}$, say $\bar{F}_{S}^{N S}$. The quantity $\eta$ below is the ratio between the standard deviation and the mean value of $\bar{F}_{S}^{N S}$, and it can be easily determined for each CMIP5 model.

To meaningfully define an F-type transition for a CMIP5 model time series, we recall the results for the box model in chapter 5 . Here, the strength of the AMOC was represented as the deep water flow in the North Atlantic (or downwelling). The F-type AMOC transitions were the consequence of a temporary cessation of the downwelling, without any subsequent reversal of the circulation. In a more realistic model, one can identify these transitions with the occurrence of extreme events in the AMOC time series, defined as values extending below a certain threshold. Drijfhout et al. [2015] used a similar approach to detect abrupt climate changes in CMIP5 models.

In the results below, the threshold used is $2 \sigma$, where $\sigma$ is standard deviation of the $\Psi_{M}^{c o r}$ time series. This choice seems to be a good compromise between two conditions that need to hold: on one hand, the threshold needs to be far enough from the mean, to guarantee that the occurrence of extreme events is well represented by a homogeneous Poisson process if the transitions are rare enough (formally when the threshold goes to infinity). On the other hand, enough values need to extend below the threshold, so that the analysis is statistically robust. Another precaution that needs to be taken is the fact that the events must be independent from each other: for this purpose, we clustered the events, using a 10-year window. Transition rates $(\lambda)$ are computed, for each time series, by counting the number of extreme events per unit of time. The transition probabilities over a time interval $T$ are then obtained using the following formula [Coles et al., 2001]

$$
P(T)=1-e^{-\lambda T},
$$

where, in most cases, we take $T=100$ years.

Values of $M_{o v}$ of the CMIP5 models were provided by Mecking et al. [2017], who calculated $M_{o v}$ at $34^{\circ} \mathrm{S}$ from historical simulations (in the period 1960-1989). Since the strength of the AMOC did not vary considerably during such time period, we assume that the corresponding values of $M_{o v}$ from control simulations do not differ much from the values in [Mecking et al., 2017]. This was confirmed for two of the models: we calculated $M_{o v}$ for pre-industrial control simulations from MIROC5 and MRI-CGCM3. In both cases, the absolute error in the estimation of $M_{o v}$ is about $0.02 \mathrm{~Sv}$. Table (6.1) contains the $M_{o v}$ values of the models considered: 
four of them are positive, which would indicate [de Vries and Weber, 2005] that the AMOC is in a single equilibrium regime and hence no noise-induced transitions are expected.

Interrelations between extremes in the strength of the AMOC and in the surface temperatures of the North Atlantic regions are studied by means of the Event Synchronisation (ES) algorithm, conceived by Quiroga et al. [2002] and improved by Malik et al. [2010]. The algorithm works as follows: consider two time series, labelled with indices $i$ and $j$, and a set of events occurring at time $t_{l}^{i}$, with $l=1,2, \ldots, s_{i}$ for the time series $i$ and at time $t_{m}^{j}$, with $m=1,2, \ldots, s_{j}$ for the time series $j$, respectively. Two events $l$ and $m$ are considered to be synchronised within a time lag $\pm \tau_{l m}^{i j}$ if $0<t_{l}^{i}-t_{m}^{j}<\tau_{l m}^{i j}$, where $\tau_{l m}^{i j}=\min \left\{t_{l+1}^{i}-t_{l}^{i}, t_{l}^{i}-t_{l-1}^{i}, t_{m+1}^{j}-\right.$ $\left.t_{m}^{j}, t_{m}^{j}-t_{m-1}^{j}\right\} / 2$. The synchronisation index for the two events is defined as:

$$
J_{l m}^{i j}= \begin{cases}1 & \text { if } 0<t_{l}^{i}-t_{m}^{j}<\tau_{l m}^{i j}, \\ 1 / 2 & \text { if } t_{l}^{i}=t_{m}^{j}, \\ 0 & \text { otherwise }\end{cases}
$$

Now it is possible to count the number of times two events occurring in the two time series are synchronised (with the event in $i$ preceding the one in $j$ ):

$$
c^{i j}=\sum_{l=1}^{s_{i}} \sum_{m=1}^{s_{j}} J_{l m}^{i j}
$$

and vice versa $c^{j i}$. The strength of the synchronisation is defined as

$$
Q^{i j}=\frac{c^{i j}+c^{j i}}{\sqrt{\left(s_{i}-2\right)\left(s_{j}-2\right)}}
$$

and normalised, such that $0 \leq Q^{i j} \leq 1$, for each pair of time series $i$ and $j \cdot Q^{i j}=1$ means full synchronisation (i.e. between a time series with itself), while $Q^{i j}=0$ indicates absence of synchronisation. With the algorithm, it is also possible to check the delay between the events: this is measured by calculating another index

$$
q^{i j}=\frac{c^{i j}-c^{j i}}{\sqrt{\left(s_{i}-2\right)\left(s_{j}-2\right)}} .
$$

When $q^{i j}$ is positive (negative), the events in the time series $j$ precede (follow) the events in the time series $i$.

\subsection{Results}

In Fig. 6.1(a), the time series of the AMOC strength $\Psi_{M}$ and the Ekman transport $\Psi_{E}$ at $26^{\circ} \mathrm{N}$ are shown for the MIROC5 model. The time series were yearlyaveraged and detrended by removing the first EOF (Empirical Orthogonal Function) obtained with the Singular-Spectrum Analysis (SSA)[Von Storch and Zwiers, 
Chapter 6. Noise-induced transitions of the Atlantic Meridional Overturning Circulation in CMIP5 models

2001]. SSA has proven to be an effective way to make sure that trends are removed from the time series: the stationarity is a necessary requirement in order to investigate extreme events. It is clear that several of the extreme events occurring in the

(a)
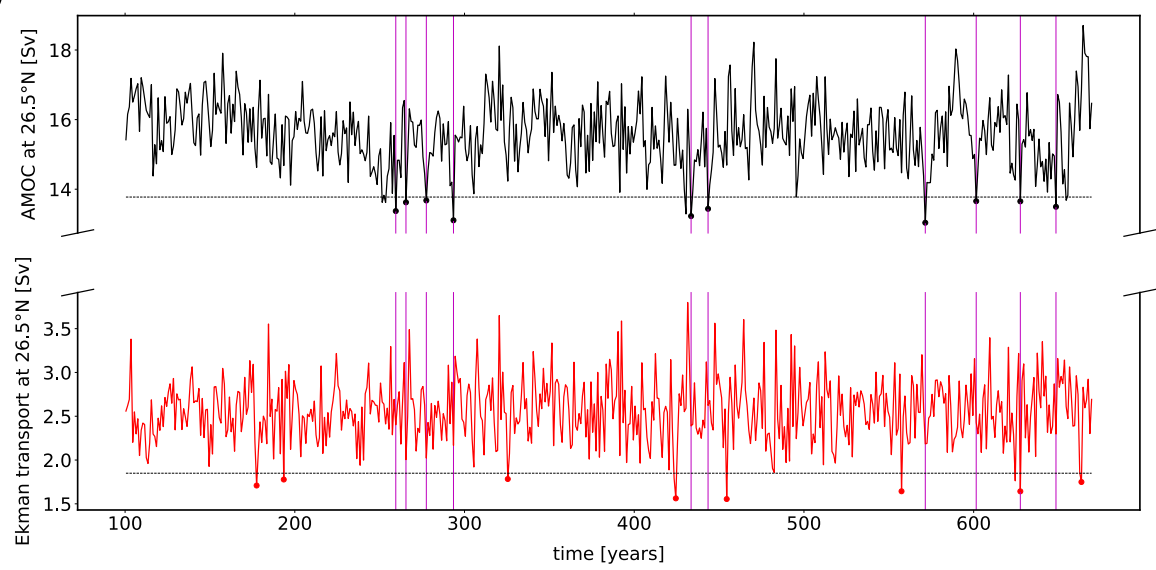

(b)

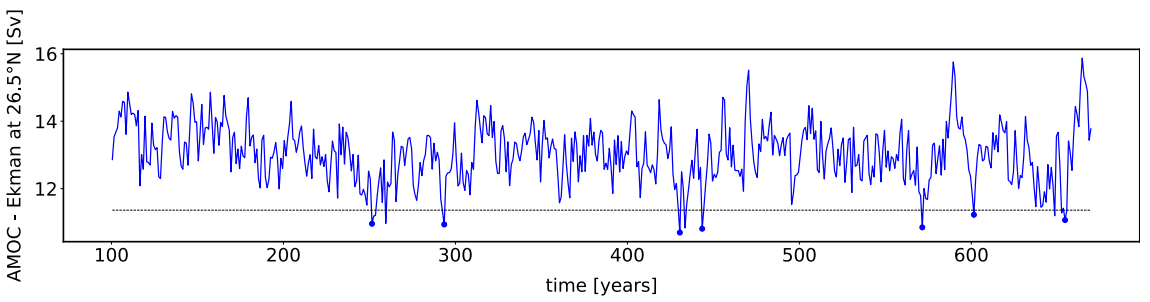

Figure 6.1: (a) Yearly-averaged time series of $\Psi_{M}$, i.e. the maximum value of the AMOC at $26.5^{\circ} \mathrm{N}$ (black) and of $\Psi_{E}$, i.e. the zonally integrated Ekman transport (red), respectively, in the pre-industrial control simulation of the model MIROC5. The dashed horizontal lines indicate, for each time series, the lower bound of the $2 \sigma$ confidence interval around the mean. The disks identify the time points at which extreme events occur, defined as the values extending below the threshold. Note that some of the values are not marked, because of the (10-year) clustering. The magenta vertical lines are drawn in correspondence with the extreme events of $\Psi_{M}$ on top of both the time series. In this way, it is possible to qualitatively check whether a certain extreme event in the AMOC is related with anomalies in the windstress variations. (b) The difference time series $\Psi_{M}^{\text {cor }}=\Psi_{M}-\Psi_{E}$.

AMOC cannot be attributed to extremes in the Ekman transport. Therefore, such events are interpreted as being buoyancy-induced and to be able to isolate these transitions we use (as mentioned in section 6.2) the time series $\Psi_{M}^{\text {cor }}=\Psi_{M}-\Psi_{E}$ (Fig. 6.1(b)).

Extreme events (or transitions) like the ones highlighted in Fig. 6.1(b), extending below the $2 \sigma$ threshold, can be found in all the CMIP5 models investigated here. Using the procedure described in the previous section, we are able to compute, for each model, the transition probability of such events; the values are 
shown in Table 6.1. At first glance, it is surprising that, although the models show very similar values of noise and transition probability, their values of $M_{o v}$ are quite different. In particular, four of the models present a positive $M_{o v}$, which would indicate that the AMOC is in a single equilibrium regime, hence unable to undergo transitions.

(a)
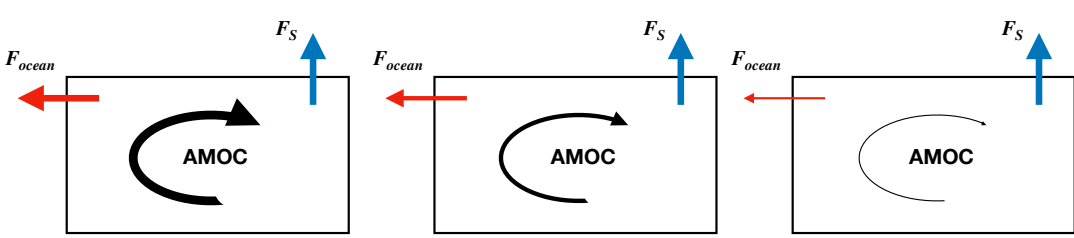

(b)
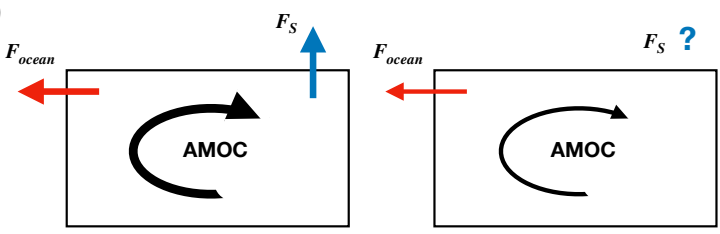

(c)
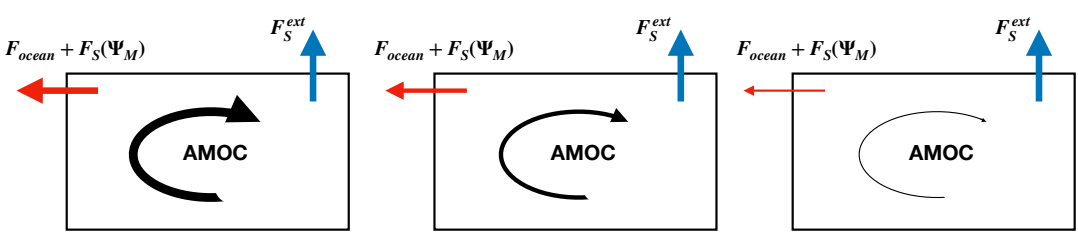

Figure 6.2: (a) Illustration of the positive feedback mechanism established with negative $M_{o v}$, in a ocean-only model. In the first figure, the AMOC is in equilibrium and it transports freshwater $\left(F_{\text {ocean }}\right)$ outside of the Atlantic ocean; $F_{S}$ represents the constant net freshwater flux between ocean and atmosphere. In the second figure, the AMOC weakens due to a perturbation: therefore, less freshwater is transported outside of the basin ( $F_{\text {ocean }}$ decreases). As a consequence, the basin becomes fresher and the AMOC further weakens, as depicted in the third figure. (b) Same situation, for a coupled model: the freshwater flux is no longer constant, therefore a weakening of the AMOC results in a change in its magnitude. Hence, no conclusions can be drawn about a potential feedback mechanism. (c) Same situation, for a coupled model, with $M_{o v}^{\text {cor }}<0$. The component of the atmospheric freshwater flux dependent on the AMOC strength $F\left(\Psi_{M}\right)$ is included in the definition of $M_{o v}$ and $F_{S}^{\text {ext }}$ is constant. Hence, the original idea behind $M_{o v}$ is retrieved.

While there could be several other reasons for this result, we interpret it here as a indication that $M_{o v}$ is not an adequate indicator of the multiple equilibrium regime in climate models. To explain our idea for a correction, we recall the physics behind $M_{o v}$ (see Fig. 6.2(a) for an illustrative scheme). When $M_{o v}$ is negative, the overturning circulation transports freshwater out of the Atlantic basin ( $F_{\text {ocean }}$ in the figure): if a perturbation is introduced in the system, such that the AMOC weak- 
ens, less freshwater is transported out of the basin; therefore, the Atlantic Ocean becomes fresher, which, in turn, further weakens the AMOC. When $M_{o v}$ is positive, a weakening of the AMOC results in a saltier basin, which has the effect of stabilising the circulation. In other words, a positive (negative) feedback mechanism on the AMOC strength is established when $M_{o v}$ is negative (positive). However, the mechanism explained above is valid only provided that atmospheric fluxes are not affected by the AMOC. This assumption is certainly valid for ocean-only models, where fixed surface fluxes are prescribed. On the other hand, when coupled models are taken into consideration, the atmospheric feedbacks cannot be ignored [den Toom et al., 2012]: a weakened AMOC results in a variation in the surface freshwater flux over the Atlantic ocean (Fig. 6.2(b)). An improved indicator for the AMOC stability needs to take such feedbacks into consideration [Gent, 2018].

Corrections to $M_{o v}$ due to salinity biases in CMIP5 models [Liu et al., 2017] have already been suggested [Mecking et al., 2017], but do not really take into account the effect of the AMOC on the freshwater flux. In the following, we take a bold step to develop a correction of $M_{o v}$ by making use of the relation between transition probabilities, freshwater noise and $M_{o v}$ as determined in the box model in 3.1, where $M_{o v}$ was a perfect indicator of the multiple equilibrium regime. It is known that box models can quite accurately represent AMOC transitions [Wood et al., 2019]. Although this approach is quite a leap, fortunately there is an interesting check at the end on the consistency of the procedure.
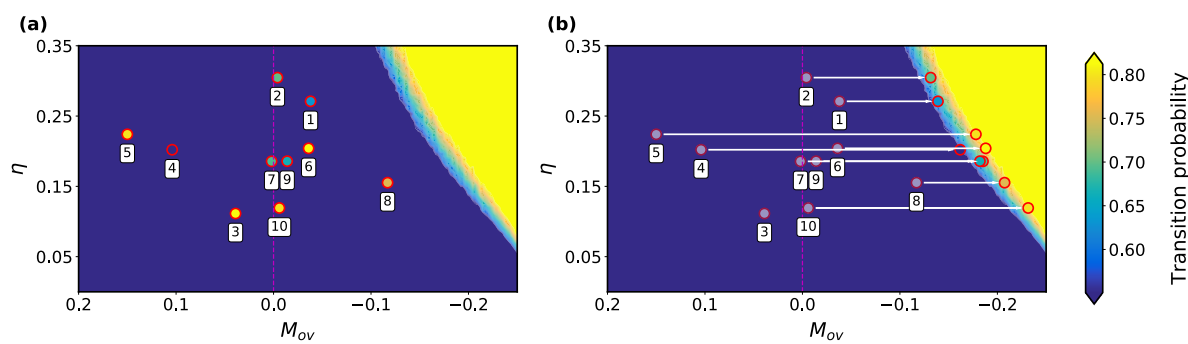

Figure 6.3: (a) Transition probabilities of the AMOC transitions in 100 years, adapted from Fig. 5.4, as function of $M_{o v}$ and atmospheric noise $\eta$. The dashed magenta line indicates $M_{o v}=0$, which is supposed to separate the monostable from the bistable regions. On the top of the contour map, circles were drawn in correspondence of the $M_{o v}$ values and the noise amplitudes calculated from the models (5th and 6th columns of Table 6.1, respectively). The transition probabilities calculated from the models, indicated by the colors of the coloured circles, do not match with the ones predicted by the box model. (b) The circles in (a) were shifted horizontally, by correcting the values of $M_{o v}$ with the procedure described in the text. The values (based on the box model) of the transition probability were calculated for each value of the parameters $\left(M_{o v}, \eta\right)$ and the $M_{o v}$ of each CMIP5 model has been corrected, such that the calculated transition probability fits the one in the box model.

As expected, the probability of F-type transitions in the previous chapter was found to be higher with lower (more negative) values of $M_{o v}$ and with higher 
noise amplitudes. Based on these results, we calculate the value of $M_{o v}$ that each CMIP5 model should have, based on the values of noise and transition probabilities calculated. This is done by inverting the map in Fig. 5.4 and interpolating $M_{o v}$ on a grid constructed with the other two variables (the atmospheric noise $\eta$ and the transition probability $P$ ). The corrected values of $M_{o v}$ are shown in the last column of Table 6.1. A graphical representation of the method is shown in Fig. 6.3. Note that the correction for the model CNRM-CM5-2 is missing: this is due to the fact that the corrected value of $M_{o v}$ should be less than $-0.25 \mathrm{~Sv}$; unfortunately, the actual value cannot be obtained with the method previously described, as in the box model, transition probabilities were not calculated for such large negative values of $M_{o v}$.

\begin{tabular}{cccccccc}
\hline \# model & Model name & Institute ID & $\Psi_{M}$ & $M_{o v}^{34^{\circ} S}(\mathrm{~Sv})$ & $\eta$ & $P$ & $M_{o v}^{\text {cor }}(\mathrm{Sv})$ \\
\hline \hline 1 & ACCESS1-0 & CSIRO-BOM & 14.7 & -0.038 & 0.27 & 0.63 & -0.14 \\
\hline 2 & ACCESS1-3 & CSIRO-BOM & 16.5 & -0.004 & 0.30 & 0.70 & -0.13 \\
\hline 3 & CNRM-CM5-2 & CNRM-CERFACS & 15.7 & +0.039 & 0.11 & 0.81 & \\
\hline 4 & GFDL-CM3 & NOAA GFDL & 20.6 & +0.104 & 0.20 & 0.55 & -0.16 \\
\hline 5 & GFDL-ESM2M & NOAA GFDL & 22.6 & +0.150 & 0.22 & 0.80 & -0.18 \\
\hline 6 & MIROC5 & MIROC & 15.5 & -0.036 & 0.20 & 0.81 & -0.19 \\
\hline 7 & MPI-ESM-LR & MPI-M & 18.9 & +0.002 & 0.19 & 0.69 & -0.19 \\
\hline 8 & MPI-ESM-MR & MPI-M & 16.6 & -0.117 & 0.16 & 0.75 & -0.21 \\
\hline 9 & MPI-ESM-P & MPI-M & 18.3 & -0.014 & 0.19 & 0.66 & -0.18 \\
\hline 10 & MRI-CGCM3 & MRI & 14.4 & -0.006 & 0.12 & 0.80 & -0.23 \\
\hline
\end{tabular}

Table 6.1: Table containing the list of the models used in this study. Each model is identified with a certain number, which is used in the following figures to refer to it. The other columns of the table represent, respectively, the AMOC strength, the value of $M_{o v}$ obtained by Mecking et al. [2017], the noise and the transition probability calculated with the procedures described in section 6.2, and the corrected values of $M_{o v}^{\text {cor }}$.

The discrepancy between the original values of $M_{o v}$ and their corrected values is here interpreted as being due to the presence of atmospheric feedbacks on the AMOC. Consider a generic General Circulation Model (GCM) and its freshwater budget in the Atlantic Ocean. In equilibrium, this budget relates the integrated freshwater flux over the Atlantic basin, including the Arctic Ocean, $\left(F_{S}\right)$ with the freshwater transports by the AMOC and the horizontal gyre circulation through [de Vries and Weber, 2005]:

$$
F_{S}=M_{o v}+M_{a z},
$$

where $M_{a z}$ represents the freshwater transport by the southern Subpolar Gyre circulation. In eq. (6.3.1), we have neglected small-scale mixing contributions and Bering Strait transport as these terms are usually much smaller than the others [Drijfhout et al., 2011]. Several studies [Cimatoribus et al., 2012] have shown that the net evaporation over the Atlantic basin depends on the strength of the AMOC, 
Chapter 6. Noise-induced transitions of the Atlantic Meridional Overturning Circulation in CMIP5 models

i.e., a lower evaporation is associated with a weaker AMOC. This motivates to split the term $F_{S}$ into two contributions:

$$
F_{S}=F_{S}\left(\Psi_{M}\right)+F_{S}^{e x t},
$$

where the first term in the right hand side represents the dependence on the AMOC strength $\Psi_{M}$, whereas the second term is the net evaporation over the Atlantic Ocean in the absence of an AMOC.

A corrected value of $M_{o v}^{\text {cor }}$ should take into account the effect of the variation of the AMOC on the transport of freshwater inside/outside the basin, hence

$$
M_{o v}^{c o r}+M_{a z}=F_{S}^{e x t} .
$$

Therefore, using the relations (6.3.1) and (6.3.2), we obtain

$$
M_{o v}^{c o r}=M_{o v}-F_{S}\left(\Psi_{M}\right) .
$$

An illustration of the mechanism is given in Fig. 6.2(c): when $M_{o v}^{c o r}$ is negative, it cannot be stated whether the AMOC transports freshwater outside or inside the Atlantic basin (the direction of $F_{\text {ocean }}$ is unknown). However, if the component of the atmospheric freshwater flux dependent on the AMOC strength $F\left(\Psi_{M}\right)$ is included in the definition of $M_{o v}$, we can imagine as if a net freshwater transport was flowing outside of the basin, partly via the southern border of the Atlantic ocean, and partly through the atmosphere $\left(F_{\text {ocean }}+F\left(\Psi_{M}\right)\right)$. When the AMOC weakens due to a perturbation, this net transport decreases, while $F_{S}^{\text {ext }}$ remains constant, by definition. Therefore, the Atlantic ocean becomes fresher, which, in turn, makes the overturning circulation weaker. One could in principle extrapolate the dependence of $F_{S}$ on the AMOC strength $\Psi_{M}$ using additional CMIP5 model simulations and calculate the corresponding value of $M_{o v}$ to determine $M_{o v}^{c o r}$. Unfortunately, such results are not available for CMIP5 models, and in fact an enormous computational effort would be required to compute all these equilibrium solutions.

However, and here comes an internal check of the procedure, it is expected that the different CMIP5 models should have about the same $F_{S}^{\text {ext }}$. If transition probabilities were not connected to the correct stability indicator, here proposed to be $M_{o v}^{c o r}$, then there would be an enormous spread in $F_{S}^{\text {ext }}$ values. From the CMIP5 models, we can evaluate $F_{S}^{e x t}$ from (combining (6.3.2) and (6.3.4))

$$
F_{S}^{e x t}=F_{S}+\Delta M_{o v}
$$

where $\Delta M_{o v}=M_{o v}^{c o r}-M_{o v}$ and $F_{S}$ is just the surface freshwater flux calculated from the control simulation. The results (Fig. 6.4) are quite astonishing as the mean value of $F_{S}^{\text {ext }}$, averaged over the 10 models, is $0.20 \mathrm{~Sv}$, with a standard deviation of only $0.06 \mathrm{~Sv}$ ! This result is highly nontrivial as the correction $M_{o v}^{\text {cor }}$ is only based on the transition probabilities. We see this as an indication of an internal consistency of the, admittedly bold, assumptions made. 


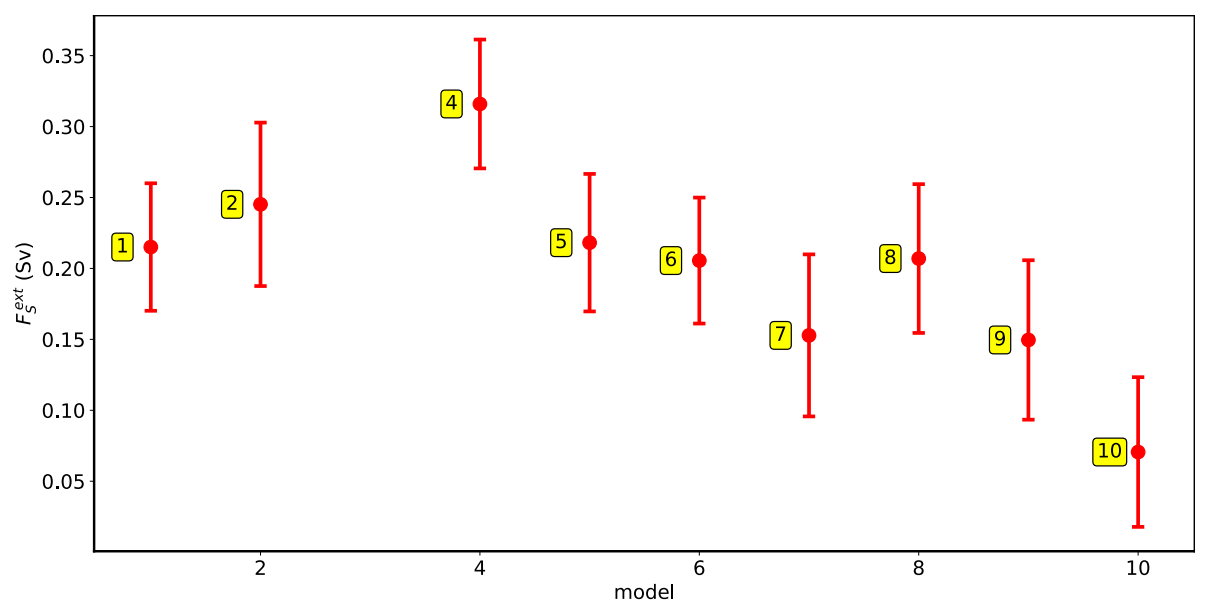

Figure 6.4: $F_{S}^{\text {ext }}$, computed for each CMIP5 model with the procedure explained in the text. The error bars represent the confidence interval of one standard deviation around the mean values, calculated for the original time series $F_{S}$.

In order to investigate the consequences of the occurrence of AMOC extremes for the surface temperatures of both land and ocean areas in the North Atlantic region, we selected three areas: Western Europe, Greenland and the Atlantic Subpolar Gyre (Fig. 6.5). The time series obtained by integrating the surface temperature field over the three subregions were yearly-averaged and detrended. Surface temperatures differ from SSTs only when sea ice is present but when the data is yearly-averaged, the differences in temperature anomalies are very small (not shown). The time series for the temperature anomalies in the three regions, once again for the model MIROC5, are shown in Fig. 6.6, together with the $\Psi_{M}^{c o r}$ time series. Several of the temperature anomalies seem to be correlated with extremes in the AMOC. In order to give a statistical measure of such correlation, for each subregion, we calculate a synchronisation index between the extreme events in the AMOC and those in the surface temperature, using the Event Synchronisation (ES) algorithm. This method is preferred over more traditional correlation methods, since our focus is on the extreme events, and not on the whole time series. Moreover, the advantage of ES is that it allows to study interrelations between series of non-Gaussian data or data with heavy tails [Malik et al., 2010, Stolbova et al., 2014]. The algorithm, is applied to two time series (indicated as $i$ and $j$, respectively): the time series $i$ was always chosen as $\Psi_{M}^{c o r}$ (top panel time series in Fig. 6.6) and $j$ was varied between the temperature anomaly time series in the three subregions (the other three time series in Fig. 6.6). Fig. 6.7 shows the results of the strength of the synchronization $Q$ and and the lag $q$, calculated for each one of the 10 CMIP5 models under consideration. $Q$ varies substantially between the different models, with a maximum value of 0.72 for the temperature anomalies 
Chapter 6. Noise-induced transitions of the Atlantic Meridional Overturning Circulation in CMIP5 models

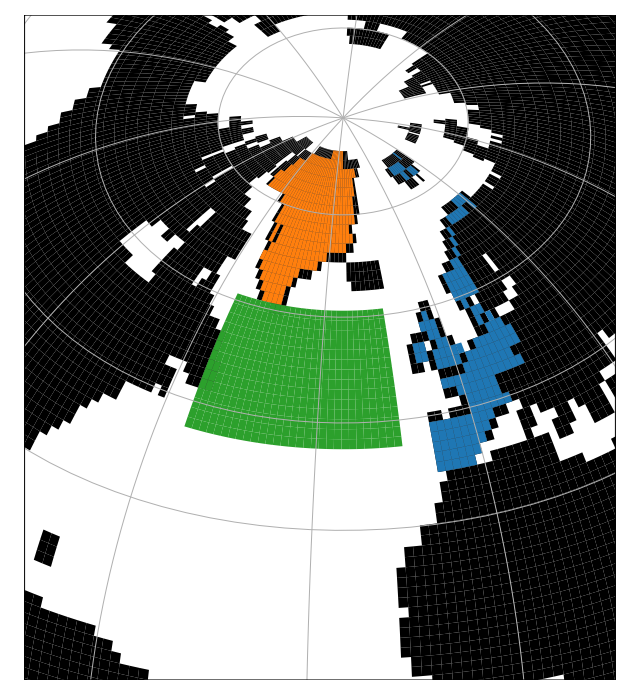

Figure 6.5: Masks corresponding to three subregions of the North Atlantic: Western Europe, Greenland and Subpolar Gyre $\left(\left[40^{\circ} \mathrm{N}, 60^{\circ} \mathrm{N}\right] \times\left[55^{\circ} \mathrm{W}, 15^{\circ} \mathrm{W}\right]\right)$.

of the Subpolar Gyre in the model MRI-CGCM3, and a minimum of zero. Overall, the temperatures are considerably affected by the AMOC. The lags calculated with the index $q$ are in almost all the cases negative, i.e. the temperature changes lag the AMOC changes. Furthermore, for the Subpolar Gyre, models with lower values of $M_{o v}^{c o r}$, which supposedly have a less stable AMOC, show higher event synchronisation between extremes in the AMOC and temperature.

\subsection{Summary and discussion}

In this chapter, we analysed noise-induced transitions of the AMOC in an ensemble of CMIP5 models, and investigated the consequences of such transitions for the surface temperatures of the North Atlantic region. In each of the CMIP5 models, a considerable number of extreme AMOC events occur, events that cannot be attributed to the wind-stress variability, as measured through the Ekman transport. Transition probabilities were calculated through a threshold measure of $2 \sigma$ around the mean.

Models simply show too many AMOC transitions, then what is expected based on their $M_{o v}$ value; therefore, we proposed a correction to $M_{o v}$. This new indicator, $M_{o v}^{c o r}$, was calibrated with the transition probabilities as obtained from a box model of the AMOC (see previous chapter). Although this was a bold step, internal consistency was demonstrated by the small spread in the integrated surface freshwater flux $F_{S}^{e x t}$ which would arise under the absence of an AMOC. The average value obtained for $F_{S}^{\text {ext }}$ from the 10 CMIP5 models was $0.20 \pm 0.06 \mathrm{~Sv}$. 

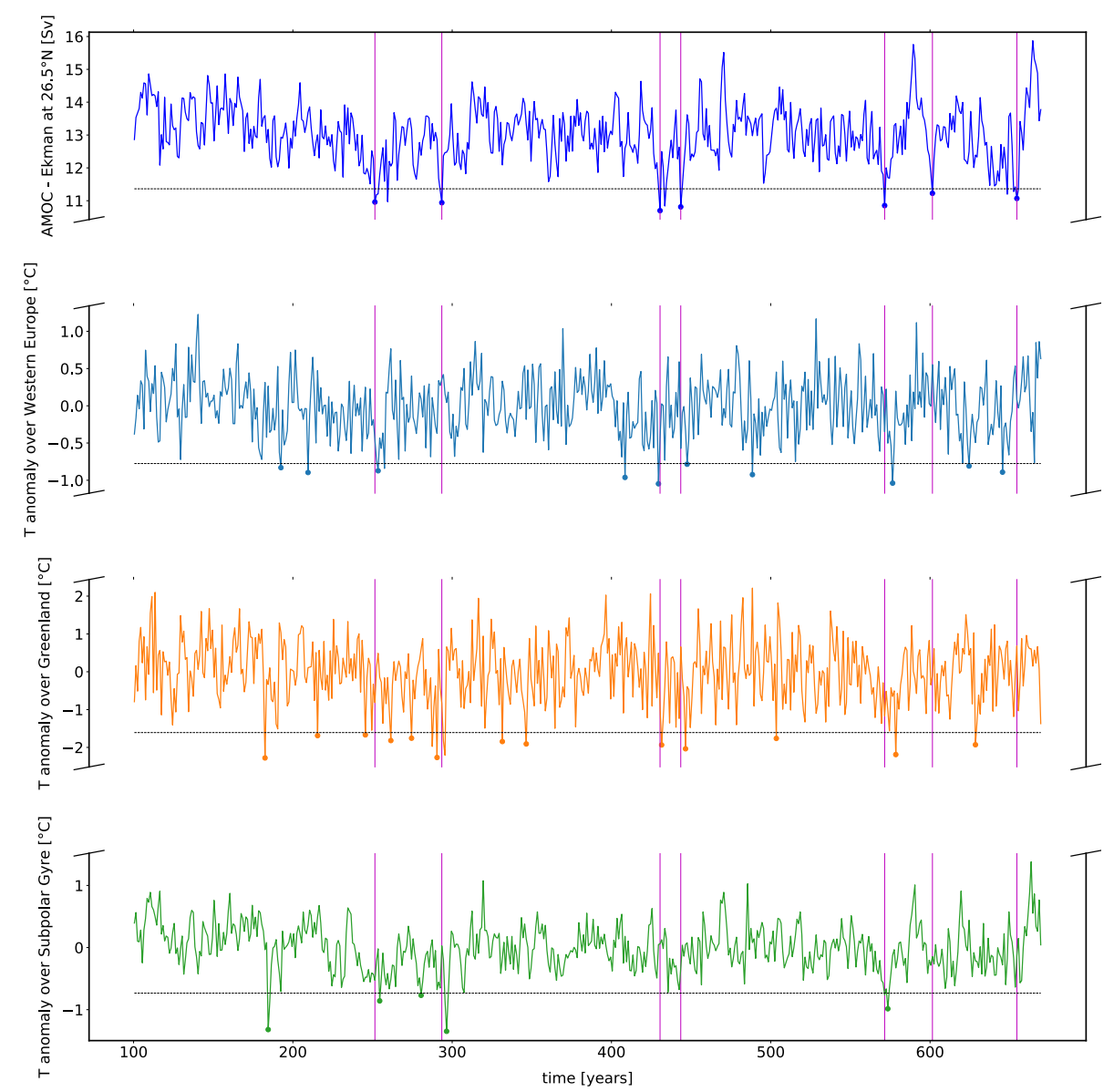

Figure 6.6: The upper panel shows the yearly-averaged time series of $\Psi_{M}^{\text {cor }}$, i.e. the buoyancy-driven component of the AMOC, in the pre-industrial control simulation of the model MIROC5. The dashed horizontal line indicates the lower bound of the $2 \sigma$ confidence interval around the mean. The circles identify the time points at which extreme events occur, defined as the values extending below the confidence interval. Note that some of the values were not marked, because of the clustering. The other three panels are the SST anomalies calculated over the three subregions indicated in Fig. 6.5. The magenta vertical lines are drawn in correspondence with the extreme events of $\Psi_{M}^{c o r}$ on top of all the time series. In this way, it is possible to qualitatively check whether a certain extreme event in the AMOC is related with anomalies in the surface temperatures.

The implication of this view is that the AMOC substantially influences the Atlantic freshwater flux $F_{S}$ and that all CMIP5 models considered are effectively in the multiple equilibrium regime. This could be investigated by applying large temporary localised freshwater fluxes and the results here would imply that collapsed 
Chapter 6. Noise-induced transitions of the Atlantic Meridional Overturning Circulation in CMIP5 models

(a)

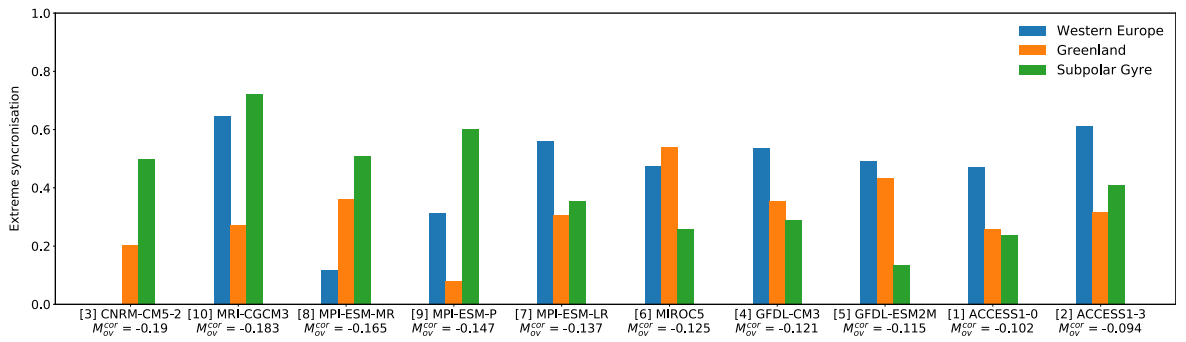

(b)

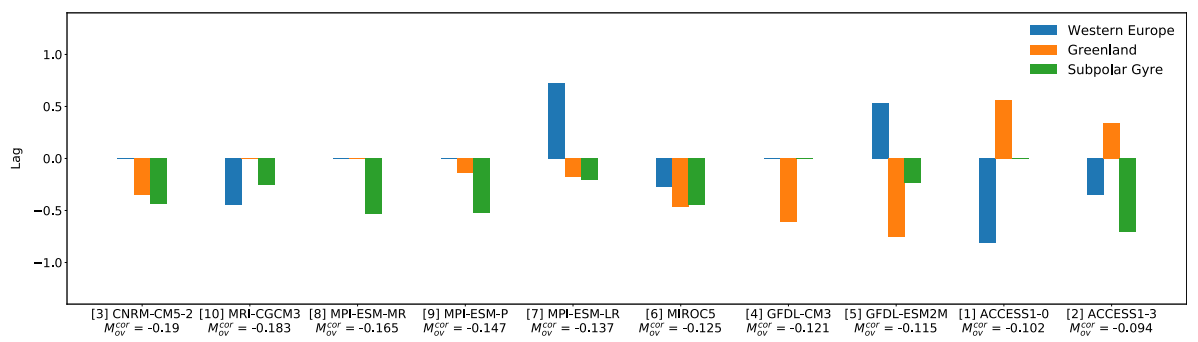

Figure 6.7: (a) Event synchronisation strength $Q$, calculated, for each CMIP5 model, between the $\Psi_{M}^{c o r}$ anomalies and the surface temperature anomalies obtained for three different subregions in the North Atlantic (Fig. 6.5). The models have been ordered, from left to right, from the lowest to the highest value of $M_{o v}^{c o r}$. (b) Lags $q$.

AMOC states can be found. It would also be interesting, but certainly out of the scope of this study, to compute $F_{S}^{e x t}$ using a coupled ocean-atmosphere model. A slab-ocean version cannot be used as in these models a so-called $Q-$ flux is prescribed, which already captures the effect of the AMOC on the freshwater flux [L'hévéder et al., 2015]. One needs to set the atmospheric buoyancy fluxes which are transferred to the ocean to zero in a fully coupled simulation. The resulting equilibrium state may be far from any historical climate state but, if so, that would precisely demonstrate the impact of the presence of the AMOC on the present-day climate state.

The values of $M_{o v}^{c o r}$ also do not dramatically change with another choice (e.g. $3 \sigma$ ) of threshold for the AMOC transitions. The transition probabilities are sensitive to changes in $M_{o v}$ : therefore, small errors in the estimation of the probabilities (still in the same order of magnitude) do not result in large errors in the estimation of $M_{o v}$.

Using event synchronisation, we studied connections between AMOC extreme events and temperatures over three North Atlantic regions in the CMIP5 models. The strength of the synchronisation substantially varies between the different models. Such differences were already found in another multi-model study, with a focus on correlations between decadal means of SST and the AMOC [Roberts et al., 
2013]. As expected, the synchronisation between AMOC and temperatures seems to occur for negative time lags, which means that the extreme events in the AMOC precede the ones in the temperatures. There is no robust connection between the stability of the AMOC, as measured by $M_{o v}^{c o r}$, and the influence of the AMOC on the surface temperatures. Indeed, many other factors, such as a different background climate state, may affect this temperature response.

\section{Acknowledgements}

We acknowledge the World Climate Research Programme Working Group on Coupled Modelling, which is responsible for CMIP, and we thank the climate modelling groups (listed in Table 6.1), for producing and making available their model output. For CMIP the U.S. Department of Energy Program for Climate Model Diagnosis and Intercomparison provides coordinating support and led development of software infrastructure in partnership with the Global Organization for Earth System Science Portals. We thank Jenny Mecking for providing the original values of $M_{o v}$ used in this work. We are also grateful to Miriam Saraceni, for her preliminary work on the correction to $M_{o v}$ and for the useful discussions. 



\section{Chapter 7}

\section{Conclusions and outlook}

In this thesis we explored the effects of high-frequency atmospheric variability, modelled as white noise, in the large-scale ocean circulation. In particular, we focused (i) on the problem of formulating a null-hypothesis for sea surface height (SSH) variability, in order to distinguish specific ocean phenomena in observational time series, and (ii) on the challenge of predicting a noise-induced collapse of the Atlantic Meridional Overturning Circulation.

\subsection{Null hypothesis for SSH variability}

In chapter 2, the first research question was addressed. By linearising the shallow water equations forced by a purely stochastic wind-stress field around its equilibrium, we obtained a linear one-dimensional stochastic model for the evolution of sea level height. A similar type of equation was suggested by Hasselmann [1976] to investigate the sea surface temperature at mid-latitudes. The main difference here is the presence of a state-dependent noise term (correlated additive and multiplicative noise, or CAM noise), arising from the interaction between the wind and the mixed layer of the ocean. Although Sura and Gille [2010] had already proposed a CAM noise stochastic model to explain SSH variability, the novelty of our study was the derivation of such model directly from the shallow water equations. Once the model was formulated, we suggested two methods for estimating its parameters from time series. The first one is an extension of what is conventionally done when estimating red-noise model parameters, that is inferring them from lag-1 autocorrelation and moments of the time series; an additional parameter is required for our model (i.e. the constant in front of the multiplicative noise term in the stochastic model), which requires the use of an additional moment of the distribution. The second method, which was eventually the preferred one, as more accurate, consists in a least-square minimisation of the difference between 
the theoretical probability density function of the model and the empirical one, directly obtained from the time series. Additionally, we introduced a measure $(\chi)$ of the validity of the stochastic model in representing the variability of the observable under study, in our case SSH, and emphasised the importance of performing a preliminary study of the distribution of the time series before proceeding with the null-hypothesis test. Finally, to show the applicability of our estimation method to observational time series, we applied the null-hypothesis test to SSH data, focusing our attention on certain locations of the ocean, where specific phenomena are expected to be detected from the analysis of SSH variability. When interpreting the power spectrum of a time series that shows a skewed distribution, we found that some peaks are significant under the red-noise test, but not significant under the CAM noise test. This indicates that using the incorrect test may lead to erroneous attribution of specific phenomena in the sea-level variability.

\subsection{Noise-induced transitions of the AMOC}

The other four chapters were dedicated to investigating atmospheric-noise-induced transitions in the Atlantic Meridional Ocean Circulation (AMOC). In chapter 3 we gave an introduction of the Trajectory-Adaptive Multilevel Sampling (TAMS) algorithm, one of the so-called rare-event algorithms [Lestang et al., 2018]. This method is particularly suitable for estimating the probability of occurrence of extreme events in a system. The great advantage of this technique, respect to brute force methods (such as Monte-Carlo simulations of big ensembles of trajectories), lies in the fact that it promotes certain trajectories and discard others, based on how close each trajectory is to the desired destination state.

In the same chapter we introduced a box model of the AMOC, originally developed by Cimatoribus et al. [2014]. The model appears suitable for addressing our research question: on one hand, enough physical processes are included, so that the effect of the overturning circulation on the freshwater transport can be distinguished from the one exerted by the subtropical gyre. On the other hand, it is still a conceptual model, and hence the computational costs required to run long simulations are very limited. In order to compute transition probabilities between the so-called 'on' and 'off' state in our model, corresponding to the present-day AMOC and to a collapsed state, respectively, we made use of TAMS. Before carrying on the simulations, we undertook a technical study of the algorithm, to make it suitable for computing transition probabilities of transitions between steady states.

In particular, in chapter 4 , we suggested some improvements to the score function, namely the function that ranks the trajectories at each time step of the algorithm and allows for the selection/discarding process. First of all, we incorporated covariance ellipsoids in the score function, in order to accurately assess the occurrence of a transition to a certain steady state. Due to the effect of the noise, indeed, we don't expect a trajectory to end up exactly in the destination equilibrium when 
undergoing a transition, but rather to wander around the steady state, and to spend most of the time in a neighbourhood of it. Covariance ellipsoids, which are based on the linearised dynamics around a steady state, are a good approximation of these neighbourhoods. The second suggested improvement to the score function revolves around the concept of transition paths. Once a typical transition path is estimated in a certain system, a score function can be designed based on it, so that trajectories in the TAMS algorithm are ranked according to how closely they follow such path. While this method may have great advantages in high-dimensional systems, where trajectories can fluctuate in many different directions, it didn't show significant improvements in the performance of the algorithm in a two-dimensional double well potential system.

For the same reason, we decided not to incorporate this second technique in TAMS, when calculating transition probabilities for noise-induced AMOC transitions, in chapter 5 . In this chapter, we finally applied TAMS to address our research question and we found that two different types of transitions may exist for the AMOC. An F-type transition is defined as a temporary shut-down of the downwelling of dense water masses in the North Atlantic: it is not a proper transition to a new equilibrium state, but rather an extreme event in the circulation; the likelihood of its occurrence in the next 100 years covers a broad range of values, depending on the amplitude of the atmospheric noise and on the background state of the system (i.e. its location in the bifurcation diagram). A complete reversal of the AMOC, referred to as an S-type transition in chapter 5, was found to be unlikely in the next century, regardless of the choice of the parameters in the model.

The connection between the AMOC background state in our box model and in high-complexity general circulation models (GCMs), such as the ones used to inform the last IPCC report, was made by means of $M_{o v}$, a well-known indicator for the stability of the AMOC. In chapter 6 , we were able to compare probabilities of occurrence of extreme events (i.e. F-type transitions) calculated directly from the GCMs and the ones obtained from the box model, by specifying $M_{o v}$ and amplitude of the atmospheric noise for each GCM. The discrepancy between the two results was attributed to the incorrectness of $M_{o v}$ as a good indicator for the AMOC stability in fully-coupled models. We therefore suggested a corrected indicator, which accounts for the presence of atmospheric feedbacks on the AMOC. Finally, we investigated the effects of these extremes on the temperatures of the North Atlantic region: the strength of the synchronization between extreme events in the circulation and in the temperatures appears to vary substantially between the different models.

\subsection{Future perspectives}

Several potential lines of future research can be defined based on our results. With regard to formulating null-hypotheses for observables of the climate system, the example of SSH suggests that a red-noise test is not always the most appropriate 
choice, despite being widely used and straightforward to perform. More complicated stochastic models can serve to define null hypotheses for different variables, especially when the associated distributions substantially deviate from a Gaussian behaviour. To this extent, the estimation of parameters proposed in chapter 2 , based on the comparison between the theoretical and the empirical PDFs, appears to be quite general, as long as a Fokker-Planck equation can be solved to obtain an analytical result for the distribution. Furthermore, in case several stochastic models are formulated, the $\chi$-squared measure can be used to assess the validity of one model respect to the other in representing the process under investigation.

The method developed to estimate the transition probability of a noise-induced collapse of the AMOC in the box model can certainly be extended to other models higher-up in the hierarchy. An example in this direction was made by Baars et al. [2019], who computed transition probabilities for a spatially two-dimensional model of the AMOC: a projected version of the TAMS algorithm was developed there, in order to overcome the computational obstacles arising from the high-dimensional nature of the system. More research is needed to make these projection methods more accurate and suitable for fully-coupled GCMs. Furthermore, incorporating typical transition paths in the definition of the score function, when computing transitions for these models, may improve the efficiency of the TAMS algorithm and simplify the computations. Laurie and Bouchet [2015] studied rare transitions in the two-dimensional barotropic quasi-geostrophic equations and were able to determine most likely transition paths using minimum action methods. It would certainly be interesting to apply their method to the AMOC model in [Baars et al., 2019] and, as second step, try to implement the score function in TAMS with the information gained on the typical transition path. 


\section{Bibliography}

Applebaum, D. (2009). Lévy processes and stochastic calculus. Cambridge university press.

Arrhenius, S. (1889). Über die Reaktionsgeschwindigkeit bei der Inversion von Rohrzucker durch Säuren. Zeitschrift für Physikalische Chemie, 4U(1):226-248.

Ashwin, P., Wieczorek, S., Vitolo, R., and Cox, P. (2012). Tipping points in open systems: bifurcation, noise-induced and rate-dependent examples in the climate system. Philosophical Transactions of the Royal Society A: Mathematical, Physical and Engineering Sciences, 370(1962):1166-1184.

Baars, S. (2019). Numerical methods for studying transition probabilities in stochastic ocean-climate models. PhD Thesis, University of Groningen.

Baars, S., Castellana, D., Wubs, F. W., and Dijkstra, H. A. (2019). Application of adaptive multilevel splitting to high-dimensional dynamical systems. Manuscript under review.

Baars, S., Viebahn, J., Mulder, T., Kuehn, C., Wubs, F., and Dijkstra, H. (2017). Continuation of probability density functions using a generalized lyapunov approach. Journal of Computational Physics, 336:627-643.

Bakke, J., Lie, O., Heegaard, E., Dokken, T., Haug, G. H., Birks, H. H., Dulski, P., and Nilsen, T. (2009). Rapid oceanic and atmospheric changes during the Younger Dryas cold period. Nature Geoscience, 2(3):202-205.

Bathiany, S., Dijkstra, H., Crucifix, M., Dakos, V., Brovkin, V., Williamson, M. S., Lenton, T. M., and Scheffer, M. (2016). Beyond bifurcation: using complex models to understand and predict abrupt climate change. Dynamics and Statistics of the Climate System, 1(November):dzw004.

Beal, L., Ruijter, W. P. M., Biastich, A., and Zahn, R. (2011). On the role of the Agulhas system in ocean circulation and climate. Nature, 472(7344):429-436.

Ben-Israel, A. and Greville, T. N. (2003). Generalized inverses: theory and applications, volume 15. Springer Science \& Business Media. 
Biastoch, A., Böning, C., Getzlaff, J., Molines, J., and Madec, G. (2008). Causes of interannual-decadal variability in the Meridional Overturning Circulation of the midlatitude North Atlantic Ocean. Journal of Climate, 21(24):6599-6615.

Boettiger, C., Ross, N., and Hastings, A. (2013). Early warning signals: The charted and uncharted territories. Theoretical Ecology, 6(3):255-264.

Bos, M. S., Williams, S. D. P., Araujo, I. B., and Bastos, L. (2014). The effect of temporal correlated noise on the sea level rate and acceleration uncertainty. Geophysical Journal International, 196(3):1423-1430.

Bouchet, F. and Reygner, J. (2016). Generalisation of the Eyring-Kramers Transition Rate Formula to Irreversible Diffusion Processes. Ann. Henri Poincaré, 17(12):3499-3532.

Bouchet, F., Rolland, J., and Simonnet, E. (2019). Rare Event Algorithm Links Transitions in Turbulent Flows with Activated Nucleations. Physical Review Letters, 122(7).

Bryan, F. (1986). High-latitude salinity effects and interhemispheric thermohaline circulations. Nature, 323(6086):301-304.

Bryden, H. L., King, B. A., and McCarthy, G. D. (2011). South Atlantic Overturning Circulation at 24s. Journal of Marine Research, 69(1):38-55.

Carpenter, S. R. and Brock, W. A. (2006). Rising variance: A leading indicator of ecological transition. Ecology Letters, 9(3):311-318.

Cérou, F., Guyader, A., et al. (2016). Fluctuation analysis of adaptive multilevel splitting. The Annals of Applied Probability, 26(6):3319-3380.

Cessi, P. and Young, W. (1992). Multiple equilibria in two-dimensional thermohaline circulation. Journal of Fluid Mechanics, 241:291-309.

Charney, J. G. and Devore, J. G. (1979). Multiple flow equilibria in the atmosphere and blocking. Journal of the Atmospheric Sciences, 36(7):1205-1216.

Cheng, W., Chiang, J. C., and Zhang, D. (2013). Atlantic Meridional Overturning Circulation (AMOC) in CMIP5 models: RCP and historical simulations. Journal of Climate, 26(18):7187-7197.

Cimatoribus, A. A., Drijfhout, S. S., Den Toom, M., and Dijkstra, H. A. (2012). Sensitivity of the Atlantic Meridional Overturning Circulation to South Atlantic freshwater anomalies. Climate dynamics, 39(9-10):2291-2306.

Cimatoribus, A. A., Drijfhout, S. S., and Dijkstra, H. A. (2014). Meridional overturning circulation: Stability and ocean feedbacks in a box model. Climate Dynamics, 42(1-2):311-328. 
Clement, A. C. and Peterson, L. C. (2008). Mechanisms of abrupt climate change of the last glacial period. Reviews of Geophysics, 46(4).

Coles, S., Bawa, J., Trenner, L., and Dorazio, P. (2001). An introduction to statistical modeling of extreme values, volume 208. Springer.

Cormen, T. H., Leiserson, C. E., Rivest, R. L., and Stein, C. (2009). Introduction to algorithms. MIT press.

Cowan, G. (1998). Statistical data analysis. Oxford university press.

Crucifix, M. (2012). Oscillators and relaxation phenomena in Pleistocene climate theory. Philosophical Transactions of the Royal Society A: Mathematical, Physical and Engineering Sciences, 370(1962):1140-1165.

Cunningham, S. A., Kanzow, T., Rayner, D., Baringer, M. O., Johns, W. E., Marotzke, J., Longworth, H. R., Grant, E. M., Hirschi, J. J.-M., Beal, L. M., Meinen, C. S., and Bryden, H. L. (2007). Temporal variability of the Atlantic meridional overturning circulation at 26.5 N. Science, 317(5840):935-938.

Dakos, V., Scheffer, M., Van Nes, E. H., Brovkin, V., Petoukhov, V., and Held, H. (2008). Slowing down as an early warning signal for abrupt climate change. Proceedings of the National Academy of Sciences of the United States of America, 105(38):14308-14312.

Dansgaard, W., Johnsen, S. J., Clausen, H. B., Dahl-Jensen, D., Gundestrup, N. S., Hammer, C. U., Hvidberg, C. S., Steffensen, J. P., Sveinbjörnsdottir, A. E., Jouzel, J., and Bond, G. (1993). Evidence for general instability of past climate from a 250-kyr ice-core record. Nature, 364(6434):218-220.

Dee, D. P., Uppala, S. M., Simmons, A. J., Berrisford, P., Poli, P., Kobayashi, S., Andrae, U., Balmaseda, M. A., Balsamo, G., Bauer, P., Bechtold, P., Beljaars, A. C. M., van de Berg, L., Bidlot, J., Bormann, N., Delsol, C., Dragani, R., Fuentes, M., Geer, A. J., Haimberger, L., Healy, S. B., Hersbach, H., Hólm, E. V., Isaksen, L., Kållberg, P., Köhler, M., Matricardi, M., McNally, A. P., Monge-Sanz, B. M., Morcrette, J.-J., Park, B.-K., Peubey, C., de Rosnay, P., Tavolato, C., Thépaut, J.-N., and Vitart, F. (2011). The ERA-interim reanalysis: Configuration and performance of the data assimilation system. Quarterly Journal of the Royal Meteorological Society, 137(656):553-597.

Delsole, T. and Farrell, B. F. (1995). A stochastically excited linear system as a model for quasigeostrophic turbulence: analytic results for one- and two-layer fluids. Journal of the Atmospheric Sciences, 52(14):2531-2547.

Dijkstra, H. A. (2005). Nonlinear physical oceanography : a dynamical systems approach to the large scale ocean circulation and El Niño, volume 28. Springer Science \& Business Media. 
Dijkstra, H. A. (2007). Characterization of the multiple equilibria regime in a global ocean model. Tellus A.

Dijkstra, H. A. (2019). Numerical bifurcation methods applied to climate models: Analysis beyond simulation. Nonlinear Processes in Geophysics, 26(4):359-369.

Dijkstra, H. A. and Ghil, M. (2005). Low-frequency variability of the large-scale ocean circulation: A dynamical systems approach. Reviews of Geophysics, 43(3).

Ditlevsen, P. D. (1999). Anomalous jumping in a double-well potential. Physical Review E - Statistical Physics, Plasmas, Fluids, and Related Interdisciplinary Topics, 60(1):172-179.

Ditlevsen, P. D. and Johnsen, S. J. (2010). Tipping points: Early warning and wishful thinking. Geophysical Research Letters, 37(19):2-5.

Ditlevsen, P. D., Kristensen, M. S., and Andersen, K. K. (2005). The recurrence time of Dansgaard-Oeschger events and limits on the possible periodic component. Journal of Climate, 18(14):2594-2603.

Drijfhout, S. (2015). Competition between global warming and an abrupt collapse of the AMOC in Earth's energy imbalance. Scientific reports, 5:14877.

Drijfhout, S., Bathiany, S., Beaulieu, C., Brovkin, V., Claussen, M., Huntingford, C., Scheffer, M., Sgubin, G., and Swingedouw, D. (2015). Catalogue of abrupt shifts in Intergovernmental Panel on Climate Change climate models. Proceedings of the National Academy of Sciences of the United States of America, 112(43):E5777E5786.

Drijfhout, S. S., Weber, S. L., and van der Swaluw, E. (2011). The stability of the MOC as diagnosed from model projections for pre-industrial, present and future climates. Climate Dynamics, 37(7-8):1575-1586.

Duchez, A., Courtois, P., Harris, E., Josey, S. A., Kanzow, T., Marsh, R., Smeed, D., and Hirschi, J.-M. (2016). Potential for seasonal prediction of Atlantic sea surface temperatures using the RAPID array at $26^{\circ} \mathrm{N}$. Climate Dynamics, 46(910):3351-3370.

Dutta, P. S., Sharma, Y., and Abbott, K. C. (2018). Robustness of early warning signals for catastrophic and non-catastrophic transitions. Oikos, 127(9):12511263.

Egger, J. (1981). Stochastically Driven Large-Scale Circulations With Multiple Equilibria. Journal of the Atmospheric Sciences, 38(12):2606-2618.

Eyring, H. (1935). The activated complex in chemical reactions. The Journal of Chemical Physics, 3(2):107-115. 
Firing, Y. L. and Merrifield, M. A. (2004). Extreme sea level events at Hawaii: Influence of mesoscale eddies. Geophysical Research Letters, 31(16).

Frankignoul, C. and Hasselmann, K. (1977). Stochastic climate models, Part II Application to sea-surface temperature anomalies and thermocline variability. Tellus, 29(4):289-305.

Franzke, C. L. E., Barbosa, S., Blender, R., Fredriksen, H., Laepple, T., Lambert, F., Nilsen, T., Rypdal, K., Rypdal, M., and Scotto, M. G. (2020). The structure of climate variability across scales. Reviews of Geophysics, 58(2):e2019RG000657.

Freidlin, M. I. and Wentzell, A. D. (1984). Random Perturbations, pages 15-43. Springer US.

Ganopolski, A. and Rahmstorf, S. (2002). Abrupt Glacial Climate Changes due to Stochastic Resonance. Physical Review Letters, 88(3):4.

Gardiner, C. W. et al. (1985). Handbook of stochastic methods, volume 3. Springer Berlin.

Gent, P. R. (2018). A commentary on the Atlantic Meridional Overturning Circulation stability in climate models. Ocean Modelling, 122(June 2017):57-66.

Ghil, M. (1976). Climate Stability for a Sellers-Type Model. Journal of the Atmospheric Sciences, 33(1):3-20.

Ghil, M. (2002). Natural Climate Variability. Encyclopedia of Global Environmental Change, 1:544-549.

Glynn, P., Rubino, G., and Tuffin, B. (2009). Robustness properties and confidence interval reliability issues. Rare Event Simulation using Monte Carlo Methods, pages 63-84.

Guckenheimer, J. and Holmes, P. (2013). Nonlinear oscillations, dynamical systems, and bifurcations of vector fields, volume 42. Springer Science \& Business Media.

Harrison, C. G. A. (2002). Power spectrum of sea level change over fifteen decades of frequency. Geochemistry Geophysics Geosystems, 3(8):1-17.

Hasselmann, K. (1976). Stochastic climate models Part I. Theory. Tellus, 28(6):473-485.

Hawkins, E., Smith, R. S., Allison, L. C., Gregory, J. M., Woollings, T. J., Pohlmann, H., and De Cuevas, B. (2011). Bistability of the Atlantic Overturning Circulation in a global climate model and links to ocean freshwater transport. Geophysical Research Letters, 38(10):1-6.

Held, H. and Kleinen, T. (2004). Detection of climate system bifurcations by degenerate fingerprinting. Geophysical Research Letters, 31(23):1-4. 
Heymann, M. and Vanden-Eijnden, E. (2008). The geometric minimum action method: A least action principle on the space of curves. Communications on Pure and Applied Mathematics, 61(8):1052-1117.

Higgins, S. I. and Scheiter, S. (2012). Atmospheric $\mathrm{CO}_{2}$ forces abrupt vegetation shifts locally, but not globally. Nature, 488(7410):209-212.

Holland, W. R. (1978). The role of mesoscale eddies in the general circulation of the ocean - numerical experiments using a quasi-geostrophic model. Journal of Physical Oceanography, 8:363-392.

Hughes, C. W. and Williams, S. D. P. (2010). The color of sea level: Importance of spatial variations in spectral shape for assessing the significance of trends. Journal of Geophysical Research, 115(C10):716-18.

Huisman, S. E., den Toom, M., Dijkstra, H. A., and Drijfhout, S. (2010). An indicator of the multiple equilibria regime of the Atlantic meridional overturning circulation. Journal of Physical Oceanography, 40(3):551-567.

Huybers, P. and Curry, W. (2006). Links between annual, Milankovitch and continuum temperature variability. Nature, 441(7091):329-332.

Jackson, L. C., Kahana, R., Graham, T., Ringer, M. A., Woollings, T., Mecking, J. V., and Wood, R. A. (2015). Global and European climate impacts of a slowdown of the AMOC in a high resolution GCM. Climate Dynamics, 45(11-12):3299-3316.

Johns, W. E., Baringer, M. O., Beal, L. M., Cunningham, S. A., Kanzow, T., Bryden, H. L., Hirschi, J. J. M., Marotzke, J., Meinen, C. S., Shaw, B., and Curry, R. (2011). Continuous, array-based estimates of Atlantic ocean heat transport at $26.5^{\circ}$ N. Journal of Climate, 24(10):2429-2449.

van Kampen, N. G. (1981). Itô versus Stratonovich. Journal of Statistical Physics, 24(1):175-187.

Keller, H. B. (1977). Numerical solution of bifurcation and nonlinear eigenvalue problems. Academic Press, New York.

Kindler, P., Guillevic, M., Baumgartner, M., Schwander, J., Landais, A., and Leuenberger, M. (2014). Temperature reconstruction from 10 to 120 kyr b2k from the NGRIP ice core. Climate of the Past, 10(2):887-902.

Kloewer, M., Latif, M., Ding, H., Greatbatch, R. J., and Park, W. (2014). Atlantic Meridional Overturning Circulation and the prediction of North Atlantic sea surface temperature. Earth and Planetary Science Letters, 406:1-6.

Kramers, H. (1940). Brownian motion in a field of force and the diffusion model of chemical reactions. Physica, 7(4):284-304. 
Kuehn, C. (2012). Deterministic Continuation of Stochastic Metastable Equilibria via Lyapunov Equations and Ellipsoids. SIAM Journal on Scientific Computing, 34(3):A1635-A1658.

Langevin, P. (1908). Sur la théorie du mouvement brownien. Compt. Rendus, 146:530-533.

Lasslop, G., Brovkin, V., Reick, C. H., Bathiany, S., and Kloster, S. (2016). Multiple stable states of tree cover in a global land surface model due to a fire-vegetation feedback. Geophysical Research Letters, 43(12):6324-6331.

Latif, M., Roeckner, E., Botzet, M., Esch, M., Haak, H., Hagemann, S., Jungclaus, J., Legutke, S., Marsland, S., Mikolajewicz, U., et al. (2004). Reconstructing, monitoring, and predicting multidecadal-scale changes in the North Atlantic thermohaline circulation with sea surface temperature. Journal of Climate, 17(7):16051614.

Laurie, J. and Bouchet, F. (2015). Computation of rare transitions in the barotropic quasi-geostrophic equations. New Journal of Physics, 17(1):015009.

Lenton, T. M. (2011). Early warning of climate tipping points. Nature Climate Change, 1(4):201-209.

Lenton, T. M., Held, H., Kriegler, E., Hall, J. W., Lucht, W., Rahmstorf, S., and Schellnhuber, H. J. (2008). Tipping elements in the Earth's climate system. Proceedings of the National Academy of Sciences of the United States of America, 105(6):1786-1793.

Lestang, T., Ragone, F., Bréhier, C. E., Herbert, C., and Bouchet, F. (2018). Computing return times or return periods with rare event algorithms. Journal of Statistical Mechanics: Theory and Experiment, 2018(4).

Levermann, A. and Fürst, J. J. (2010). Atlantic pycnocline theory scrutinized using a coupled climate model. Geophysical Research Letters, 37(14).

Lin, J. W. B. and Neelin, J. D. (2002). Considerations for stochastic convective parameterization. Journal of the Atmospheric Sciences, 59(5):959-975.

Liu, W., Liu, Z., and Brady, E. C. (2014). Why is the AMOC monostable in coupled general circulation models? Journal of Climate, 27(6):2427-2443.

Liu, W., Xie, S.-P., Liu, Z., and Zhu, J. (2017). Overlooked possibility of a collapsed Atlantic Meridional Overturning Circulation in warming climate. Science Advances, 3(1):e1601666.

Livina, V. N., Kwasniok, F., Lohmann, G., Kantelhardt, J. W., and Lenton, T. M. (2011). Changing climate states and stability: From Pliocene to present. Climate Dynamics, 37(11-12):2437-2453. 
Livina, V. N. and Lenton, T. M. (2007). A modified method for detecting incipient bifurcations in a dynamical system. Geophysical Research Letters, 34(3).

Lorenz, E. N. (1963). Deterministic Nonperiodic Flow. Journal of the Atmospheric Sciences, 20(2):130-141.

Lovejoy, S. (2015). A voyage through scales, a missing quadrillion and why the climate is not what you expect. Climate Dynamics, 44(11-12):3187-3210.

L'hévéder, B., Codron, F., and Ghil, M. (2015). Impact of anomalous northward oceanic heat transport on global climate in a slab ocean setting. Journal of Climate, 28(7):2650-2664.

Malik, N., Marwan, N., and Kurths, J. (2010). Spatial structures and directionalities in Monsoonal precipitation over South Asia. Nonlinear Processes in Geophysics, 17(5):371-381.

Mann, M. E., Bradley, R. S., and Hughes, M. K. (1999). Northern hemisphere temperatures during the past millennium: Inferences, uncertainties, and limitations. Geophysical Research Letters, 26(6):759-762.

Mecking, J. V., Drijfhout, S. S., Jackson, L. C., and Andrews, M. B. (2017). The effect of model bias on Atlantic freshwater transport and implications for AMOC bi-stability. Tellus A: Dynamic Meteorology and Oceanography, 69(1):1299910.

Mecking, J. V., Drijfhout, S. S., Jackson, L. C., and Graham, T. (2016). Stable AMOC off state in an eddy-permitting coupled climate model. Climate Dynamics, 47(7):2455-2470.

Mitchell, J. M. (1976). An overview of climatic variability and its causal mechanisms. Quaternary Research, 6(4):481-493.

Monahan, A. H. (2004). A simple model for the skewness of global sea surface winds. Journal of the Atmospheric Sciences, 61(16):2037-2049.

Mudelsee, M. (2013). Climate time series analysis. Springer.

Neelin, J., Battisti, D. S., Hirst, A. C., Jin, F.-F., Wakata, Y., Yamagata, T., and Zebiak, S. E. (1998). ENSO Theory. Journal of Geophysical Research, 103:14,261-14,290.

Neelin, J. D. and Weng, W. (1999). Analytical prototypes for ocean-atmosphere interaction at midlatitudes. Part I: Coupled feedbacks as a sea surface temperature dependent stochastic process. Journal of Climate, 12(2-3):697-721.

Newman, M. (2007). Interannual to decadal predictability of tropical and North Pacific sea surface temperatures. Journal of Climate, 20(11):2333-2356. 
Newman, M., Sardeshmukh, P. D., and Penland, C. (1997). Stochastic forcing of the wintertime extratropical flow. Journal of the Atmospheric Sciences, 54(3):435455.

North, G. R., Mengel, J. G., and Short, D. A. (1983). Simple energy balance model resolving the seasons and the continents: application to the astronomical theory of the ice ages. Journal of Geophysical Research, 88(C11):6576-6586.

Palter, J. B. (2015). The role of the Gulf Stream in European climate. Annual review of marine science, 7:113-137.

Pavliotis, G. A. (2016). Stochastic processes and applications. Springer-Verlag, New York.

Pedlosky, J. (1996). Ocean Circulation Theory. Springer Science \& Business Media.

Pierini, S. (2010). Coherence resonance in a double-gyre model of the Kuroshio Extension. Journal of Physical Oceanography, 40(1):238-248.

Polo, I., Robson, J., Sutton, R., and Balmaseda, M. A. (2014). The importance of wind and buoyancy forcing for the boundary density variations and the geostrophic component of the AMOC at $26^{\circ} \mathrm{N}$. Journal of Physical Oceanography, 44(9):2387-2408.

Qiu, B. and Miao, W. (2000). Kuroshio path variations south of Japan: bimodality as a self-sustained internal oscillation. Journal Of Physical Oceanography, 30:2124-2137.

Quiroga, R. Q., Kreuz, T., and Grassberger, P. (2002). Event synchronization: a simple and fast method to measure synchronicity and time delay patterns. Physical review $E$, 66(4):041904.

Rahmstorf, S. (1996). On the freshwater forcing and transport of the Atlantic thermohaline circulation. Climate Dynamics, 12(12):799-811.

Rahmstorf, S., Box, J. E., Feulner, G., Mann, M. E., Robinson, A., Rutherford, S., and Schaffernicht, E. J. (2015). Exceptional twentieth-century slowdown in Atlantic Ocean Overturning Circulation. Nature climate change, 5(5):475.

Rahmstorf, S., Crucifix, M., Ganopolski, A., Goosse, H., Kamenkovich, I., Knutti, R., Lohmann, G., Marsh, R., Mysak, L. A., Wang, Z., et al. (2005). Thermohaline circulation hysteresis: A model intercomparison. Geophysical Research Letters, $32(23)$.

Ridley, J., Gregory, J. M., Huybrechts, P., and Lowe, J. (2010). Thresholds for irreversible decline of the Greenland ice sheet. Climate Dynamics, 35(6):10651073. 
Ritchie, P. and Sieber, J. (2016). Early-warning indicators for rate-induced tipping. Chaos: An Interdisciplinary Journal of Nonlinear Science, 26(9):93116.

Roberts, C. D., Garry, F. K., and Jackson, L. C. (2013). A multimodel study of sea surface temperature and subsurface density fingerprints of the Atlantic Meridional Overturning Circulation. Journal of Climate, 26(22):9155-9174.

Robinson, A., Calov, R., and Ganopolski, A. (2012). Multistability and critical thresholds of the Greenland ice sheet. Nature Climate Change, 2(6):429-432.

Rolland, J. and Simonnet, E. (2015). Statistical behaviour of adaptive multilevel splitting algorithms in simple models. Journal of Computational Physics, 283:541-558.

Sardeshmukh, P., Penland, C., and Newman, M. (2001). Rossby waves in a stochastically fluctuating medium. In Stochastic Climate Models, pages 369-384. Springer.

Sardeshmukh, P. D. and Sura, P. (2009). Reconciling Non-Gaussian Climate Statistics with Linear Dynamics. Journal Of Climate, 22(5):1193-1207.

Scheffer, M., Bascompte, J., Brock, W. A., Brovkin, V., Carpenter, S. R., Dakos, V., Held, H., Van Nes, E. H., Rietkerk, M., and Sugihara, G. (2009). Early-warning signals for critical transitions. Nature, 461(7260):53-59.

Serra, C., Burgueo, A., and Lana, X. (2001). Analysis of maximum and minimum daily temperatures recorded at fabra observatory (Barcelona, NE Spain) in the period 1917-1998. International Journal of Climatology, 21(5):617-636.

Smeed, D., Josey, S., Beaulieu, C., Johns, W. E., Moat, B., Frajka-Williams, E., Rayner, D., Meinen, C., Baringer, M., Bryden, H., et al. (2018). The North Atlantic Ocean is in a state of reduced overturning. Geophysical Research Letters, 45(3):1527-1533.

Smeed, D., Moat, B., Rayner, D., Johns, W., Baringer, M., Volkov, D., and FrajkaWilliams, E. (2019). Atlantic Meridional Overturning Circulation observed by the RAPID-MOCHA-WBTS (RAPID-Meridional Overturning Circulation and heatflux array-western boundary time series) array at $26^{\circ} \mathrm{N}$ from 2004 to 2018. British Oceanographic Data Centre - Natural Environment Research Council, UK.

Smeed, D. A., McCarthy, G. D., Cunningham, S. A., Frajka-Williams, E., Rayner, D., Johns, W. E., Meinen, C. S., Baringer, M. O., Moat, B. I., Duchez, A., and Bryden, H. L. (2014). Observed decline of the Atlantic Meridional Overturning Circulation 2004-2012. Ocean Science, 10(1):29-38.

Soldatenko, S. and Yusupov, R. (2017). Predictability in Deterministic Dynamical Systems with Application to Weather Forecasting and Climate Modelling. Dynamical Systems - Analytical and Computational Techniques, page 101. 
Srokosz, M., Baringer, M., Bryden, H., Cunningham, S., Delworth, T., Lozier, S., Marotzke, J., and Sutton, R. (2012). Past, present, and future changes in the Atlantic Meridional Overturning Circulation. Bulletin of the American Meteorological Society, 93(11):1663-1676.

Stocker, T. F., Qin, D., Plattner, G. K., Tignor, M. M., Allen, S. K., Boschung, J., Nauels, A., Xia, Y., Bex, V., and Midgley, P. M. (2013). Climate change 2013 the physical science basis. Working Group I Contribution to the Fifth Assessment Report of the Intergovernmental Panel on Climate Change, 9781107057:1-1535.

Stolbova, V., Tupikina, L., Bookhagen, B., Marwan, N., and Kurths, J. (2014). Topology and seasonal evolution of the network of extreme precipitation over the Indian subcontinent and Sri Lanka. Nonlinear Processes in Geophysics.

Stommel, H. (1961). Thermohaline Convection with Two Stable Regimes of Flow. Tellus B, 13(2):224-230.

Sultan, B. and Janicot, S. (2000). Abrupt shift of the ITCZ over West Africa and intra-seasonal variability. Geophysical Research Letters, 27(20):3353-3356.

Sura, P. (2002). Noise-induced transitions in a barotropic $\beta$-plane channel. Journal of the Atmospheric Sciences, 59(1):97-110.

Sura, P., Fraedrich, K., and Lunkeit, F. (2001). Regime transitions in a stochastically forced double-gyre model. J. Phys. Oceanography, 31(2):411-426.

Sura, P. and Gille, S. T. (2010). Stochastic dynamics of sea surface height variability. Journal of Physical Oceanography, 40(7):1582-1596.

Sura, P., Newman, M., and Alexander, M. A. (2006). Daily to decadal sea surface temperature variability driven by state-dependent stochastic heat fluxes. Journal of Physical Oceanography, 36(10):1940-1958.

Sura, P. and Sardeshmukh, P. D. (2008). A Global View of Non-Gaussian SST Variability. Journal Of Physical Oceanography, 38:639-647.

Taylor, K. E., Stouffer, R. J., and Meehl, G. A. (2012). An Overview of CMIP5 and Experimental Design. Bulletin of the American Meteorological Society, 93(april):485-498.

Teo, I., Mayne, C. G., Schulten, K., and Lelièvre, T. (2016). Adaptive Multilevel Splitting Method for Molecular Dynamics Calculation of Benzamidine-Trypsin Dissociation Time. Journal of Chemical Theory and Computation, 12(6):29832989.

Thompson, K. R. and Demirov, E. (2006). Skewness of sea level variability of the world's oceans. Journal of Geophysical Research: Oceans, 111(5):1-11. 
den Toom, M., Dijkstra, H. A., Cimatoribus, A. A., and Drijfhout, S. S. (2012). Effect of atmospheric feedbacks on the stability of the Atlantic Meridional Overturning Circulation. Journal of Climate, 25(12):4081-4096.

den Toom, M., Dijkstra, H. A., Weijer, W., Hecht, M. W., Maltrud, M. E., and Sebille, E. V. (2014). Response of a Strongly Eddying Global Ocean to North Atlantic Freshwater Perturbations. Journal of Physical Oceanography, 44(2):464-481.

Toom, M. D., Dijkstra, H. A., and Wubs, F. W. (2011). Spurious multiple equilibria introduced by convective adjustment. Ocean Modelling, 38(1-2):126-137.

Vellinga, M. and Wood, R. A. (2002). Global climatic impacts of a collapse of the Atlantic thermohaline circulation. Climatic Change, 54(3):251-267.

Von Storch, H. and Zwiers, F. W. (2001). Statistical analysis in climate research. Cambridge university press.

de Vries, P. and Weber, S. L. (2005). The Atlantic freshwater budget as a diagnostic for the existence of a stable shut down of the Meridional Overturning Circulation. Geophysical Research Letters, 32(9):1-4.

Wasserman, L. (2013). All of statistics: a concise course in statistical inference. Springer Science \& Business Media.

Weijer, W., Cheng, W., Drijfhout, S. S., Fedorov, A. V., Hu, A., Jackson, L. C., Liu, W., McDonagh, E. L., Mecking, J. V., and Zhang, J. (2019). Stability of the Atlantic Meridional Overturning Circulation: A review and synthesis. Journal of Geophysical Research: Oceans, 124(8):5336-5375.

Weijer, W., Maltrud, M. E., Hecht, M. W., Dijkstra, H. A., and Kliphuis, M. A. (2012). Response of the Atlantic Ocean circulation to Greenland Ice Sheet melting in a strongly-eddying ocean model. Geophysical Research Letters, 39(9).

Weijer, W., Ruijter, W. P. M. D., and Dijkstra, H. A. (2001). Stability of the Atlantic Overturning Circulation: Competition between Bering Strait freshwater flux and Agulhas heat and salt sources. Journal of Physical Oceanography, 31(8):23852402.

Whitaker, J. S. and Sardeshmukh, P. D. (1998). A linear theory of extratropical synoptic eddy statistics. Journal of the Atmospheric Sciences, 55(2):237-258.

Wieczorek, S., Ashwin, P., Luke, C. M., and Cox, P. M. (2011). Excitability in ramped systems: The compost-bomb instability. Proceedings of the Royal Society A: Mathematical, Physical and Engineering Sciences, 467(2129):1243-1269.

Wood, R. A., Rodríguez, J. M., Smith, R. S., Jackson, L. C., and Hawkins, E. (2019). Observable, low-order dynamical controls on thresholds of the Atlantic Meridional Overturning Circulation. Climate Dynam., 53:6815-6834. 
Yin, J., Schlesinger, M. E., Andronova, N. G., Malyshev, S., and Li, B. (2006). Is a shutdown of the thermohaline circulation irreversible? Journal of Geophysical Research Atmospheres, 111(12):1-17.

Zhao, J. and Johns, W. (2014). Wind-forced interannual variability of the Atlantic Meridional Overturning Circulation at $26.5^{\circ} \mathrm{N}$. Journal of Geophysical Research: Oceans, 119(4):2403-2419. 



\section{Publications and preprints}

Castellana, D., Baars, S., Wubs, F. W., and Dijkstra, H. A. (2019). Transition probabilities of noise-induced transitions of the Atlantic Ocean circulation. Scientific Reports, 9(1):1-7.

Castellana, D., Dijkstra, H. A., and Wubs, F. W. (2018). A statistical significance test for sea-level variability. Dynamics and Statistics of the Climate System, 3(1):dzy008.

Castellana, D. and Dijkstra, H. A. (2020). Noise-induced transitions of the Atlantic Meridional Overturning Circulation in CMIP5 models. Manuscript under review.

Wang, P., Castellana, D., and Dijkstra, H. A. (2020). Improvements to the use of the Trajectory-Adaptive Multilevel Sampling algorithm for the study of rare events. Manuscript under review.

Jüling, A., Zhang, X., Castellana, D., von der Heydt, A. S., and Dijkstra, H. A. (2020). The Atlantic's freshwater budget under climate change in the Community Earth System Model with strongly eddying oceans. Manuscript under review.

Baars, S., Castellana, D., Wubs, F. W., and Dijkstra, H. A. (2019). Application of adaptive multilevel splitting to high-dimensional dynamical systems. Manuscript under review.

Dijkstra, H. A., Tantet, A., Viebahn, J., Mulder, E., Hebbink, M., Castellana, D., van den Pol, H., Frank, J., Baars, S., Wubs, F., et al. (2016). A numerical framework to understand transitions in high-dimensional stochastic dynamical systems. Dynamics and Statistics of the Climate System, 1(1). 



\section{Curriculum Vitae}

Daniele Castellana was born on 23 October 1989 in Larino, Italy and grew up in Piacenza, a small city in the middle of the Po Valley. After high school he moved to Pavia, where he completed his Bachelor in Physics, and pursued his studies with a Master in Theoretical Physics at Sapienza University, in Rome. Fascinated by statistical mechanics and dynamical system theory, and motivated by a strong personal interest in issues related to climate change, he decided to do his $\mathrm{PhD}$ in Physical Oceanography at IMAU, in Utrecht. Currently, he is exploring job opportunities outside of academia, with the aim of applying the knowledge and skills obtained during his education in a different context, that can make a direct contribution to society. 



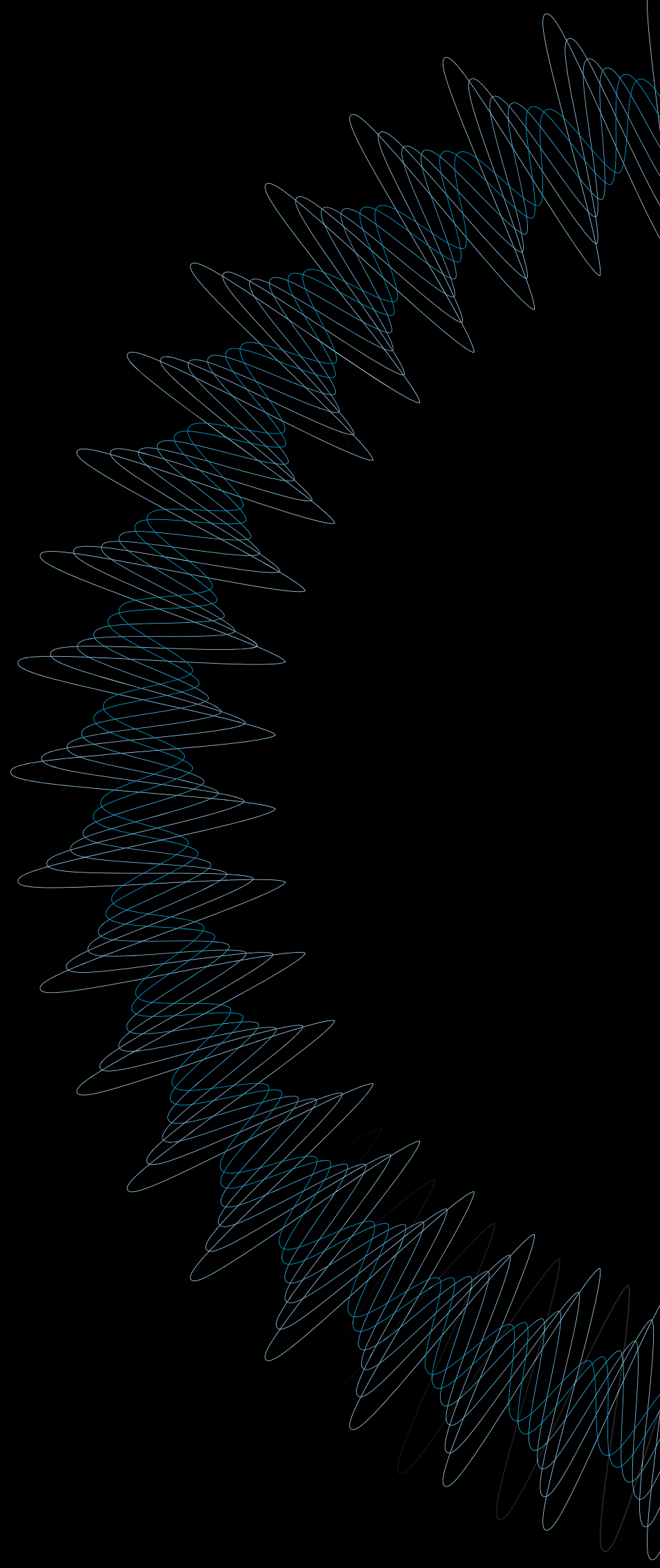

